\title{
Outer unipotent classes in automorphism groups of simple algebraic groups
}

\author{
Ross Lawther \\ DPMMS \\ University of Cambridge \\ Cambridge CB3 0WB \\ $\mathrm{UK}$ \\ ril10@cam.ac.uk
}

\author{
Martin W. Liebeck \\ Department of Mathematics \\ Imperial College \\ London SW7 2AZ \\ UK \\ m.liebeck@imperial.ac.uk
}

\author{
Gary M. Seitz \\ Department of Mathematics \\ University of Oregon \\ Eugene \\ Oregon 97403 \\ USA \\ seitz@uoregon.edu
}

May 3, 2013

\begin{abstract}
We study the unipotent elements of disconnected algebraic groups of the form $G\langle\tau\rangle$, where $G$ is a simple algebraic group in characterisctic $p$ possessing a graph automorphism $\tau$ of order $p$. We classify the unipotent classes in the coset $G \tau$ and determine the corresponding centralizers, showing that these bear a close relation to classes in a certain natural connected overgroup of $G\langle\tau\rangle$. We also obtain a formula for the total number of outer unipotent elements in the finite group $G_{\gamma}\langle\tau\rangle$, where $\gamma$ is a Frobenius morphism, analogous to the well-known Steinberg formula for the number of inner unipotent elements.
\end{abstract}

\section{Introduction}

Let $G$ be a simple algebraic group over an algebraically closed field of characteristic $p$. There is a substantial literature concerning the unipotent classes of $G$ and its finite analogues; for example, precise and detailed information on centralizers of representatives of these classes can be found in [11]. If the pair $(G, p)$ is $\left(A_{l}, 2\right),\left(D_{l}, 2\right),\left(E_{6}, 2\right)$ or $\left(D_{4}, 3\right)$ (with $G$ being either simply connected 
or adjoint in the last case), there is a graph automorphism $\tau$ of $G$ of order $p$, and the coset $G \tau$ contains elements of order a power of $p$, which can be regarded as unipotent elements. Indeed, if we take $G$ to be simply connected, then $G\langle\tau\rangle$ is contained in a larger simple algebraic group (respectively $C_{l+1}, B_{l}, E_{7}$ or $F_{4}$ ), and the $p$-elements in $G \tau$ are unipotent elements of the larger group.

This paper is a contribution to understanding the outer unipotent elements in the disconnected group $G\langle\tau\rangle$ and corresponding finite analogues $G_{\sigma}\langle\tau\rangle$ for $\sigma$ a Frobenius morphism. At the outset, it is not evident that there are only finitely many conjugacy classes of such elements under the action of $G$. This assertion can be found in [16], and an elementary proof based on the finiteness of the number of unipotent classes in $G$ is given by Fulman and Guralnick in $[6,2.6]$. In section 3 we shall use the method of [6] along with information on unipotent classes in $G$ to determine the precise number of outer unipotent classes in the finite groups and then use this information to show that there are only finitely many outer unipotent classes in $G\langle\tau\rangle$.

There is also an elegant formula (see Theorem 1.1 below) giving the total number of outer unipotent elements in the extended finite groups, which is in the spirit of Steinberg's well-known formula for the number of inner unipotent elements (see [4, Theorem 6.6.1]).

A main goal of the paper is to obtain information on the conjugacy classes and precise centralizers of the outer unipotent elements. In addition, we want to explicitly relate the outer unipotent classes of $G \tau$ to the usual unipotent classes of the simple overgroup indicated above.

For $(G, p)=\left(D_{l}, 2\right)$, we can view the group $G\langle\tau\rangle$ as the full orthogonal group, and information on classes and centralizers, in both the algebraic and finite orthogonal groups, is given in [11, Chapters 6, 7]. In this paper we therefore mainly focus on the remaining cases where $(G, p)=\left(A_{l}, 2\right),\left(E_{6}, 2\right)$ or $\left(D_{4}, 3\right)$. For the algebraic groups this was first taken up by Spaltenstein in [16] where he obtains information on conjugacy classes and dimensions of centralizers. For the finite groups $E_{6}^{\epsilon}(q) .2, D_{4}^{\epsilon}(q) .3$, and $A_{l}^{\epsilon}(q) .2$ with $l \leq 5$, information on outer classes and centralizer orders is given by Malle in [12], [13]. However, none of these papers determines the precise centralizers of the outer unipotent elements, in particular the reductive part of the centralizers.

Our aim here is to precisely identify the conjugacy classes of such elements in the algebraic groups, to determine centralizers of representatives of these classes, and to obtain similar information for the corresponding finite groups of Lie type. We achieve this in Theorems 1.2, 1.3, 1.4 and 1.5 below. All that we use from the literature above is Spaltenstein's description of classes and centralizer dimensions in the algebraic group $A_{l} .2$; however, we interpret this information within $C_{l+1}$, where it is quite natural and appears very much like corresponding results for the classical groups in characteristic 2 as developed in [11].

In the remainder of this introductory section we first set out a hypothesis which will apply throughout this paper and introduce some standard notation; 
we then state our main results. We begin with our general result on the number of outer unipotent elements in the finite groups; we then give our detailed results on conjugacy classes and centralizers separately for the $A_{l}$ case and for the $E_{6}$ and $D_{4}$ cases. Finally we describe the structure of the rest of the paper.

\subsection{Notation}

Let $G$ be a simple algebraic group over an algebraically closed field $K$ of characteristic $p$; throughout this paper we shall assume that

$$
(G, p)=\left(A_{l}, 2\right),\left(D_{l}, 2\right),\left(E_{6}, 2\right) \text { or }\left(D_{4}, 3\right),
$$

with $G$ being either simply connected or adjoint in the last case. Let $H$ be a simply connected simple algebraic group over $K$, of type $C_{l+1}, B_{l}, E_{7}$ or $F_{4}$ respectively.

Fix a maximal torus $T_{G}$ of $G$ and take root subgroups with respect to $T_{G}$; let $\Sigma$ denote the root system of $G$, with simple system $\Pi=\left\{\alpha_{1}, \ldots, \alpha_{r}\right\}$ where $r$ is the rank of $G$, and corresponding positive system $\Sigma^{+}$. For $\beta \in \Sigma$, let $X_{\beta}$ denote the corresponding root subgroup, and $x_{\beta}: K \rightarrow X_{\beta}$ be an isomorphism of algebraic groups, so that $X_{\beta}=\left\{x_{\beta}(c): c \in K\right\}$; we assume the maps $x_{\beta}$ are chosen such that the Chevalley commutator relations hold. Let $U=\prod_{\beta \in \Sigma^{+}} X_{\beta}$. To simplify notation write $u_{\beta}=x_{\beta}(1)$ for $\beta \in \Sigma$.

For $j=1, \ldots, r$ let $s_{j}=x_{\alpha_{j}}(1) x_{-\alpha_{j}}(-1) x_{\alpha_{j}}(1)$ be the standard representative in $N_{G}\left(T_{G}\right)$ of the Weyl group reflection in the $j$ th simple root. We shall write roots as linear combinations of simple roots, with the coefficients arranged in the form of the Dynkin diagram; thus for example, if $G=E_{6}$ or $D_{4}$, the highest root in $\Sigma$ is denoted ${ }_{2}^{12321}$ or $12{ }_{1}^{1}$ respectively.

Given a group $A$ and an automorphism $\theta$ of $A$, write $A_{\theta}=\left\{a \in A: a^{\theta}=a\right\}$ for the group of fixed points. Let $\tau$ be a graph automorphism of $G$ of order $p$ which permutes the elements of $\Pi$; we may assume that $x_{\alpha_{j}}(c)^{\tau}=x_{\alpha_{j} \tau}(c)$ for all $j$ and all $c \in K$ (the assumption on the isogeny type in the case $(G, p)=\left(D_{4}, 3\right)$ is required to ensure $\tau$ exists). Then if $G$ is simply connected we may regard $G\langle\tau\rangle$ as a subgroup of $H$. Elements of the coset $G \tau$ which have order a power of $p$ will be called outer unipotent elements.

Let $q$ be a power of $p$, and $\sigma$ denote the $q$-field morphism of $G$ satisfying $x_{\beta}(c)^{\sigma}=x_{\beta}\left(c^{q}\right)$ for all $\beta \in \Sigma$ and $c \in K$; let $\gamma$ denote either $\sigma$ or $\sigma \tau$. Then $\gamma$ is a Frobenius morphism of $G$. For example, if $G=E_{6}$ is adjoint we have $G_{\gamma}=\operatorname{Inndiag}\left(E_{6}^{\epsilon}(q)\right)$, with $\epsilon=1$ or -1 according as $\gamma=\sigma$ or $\sigma \tau$ (where $E_{6}^{1}(q)=E_{6}(q)$ and $\left.E_{6}^{-1}(q)={ }^{2} E_{6}(q)\right)$. Write $G_{\gamma, \tau}=\left(G_{\gamma}\right)_{\tau}$.

Given a group $A$ and an element $a$ of $A$, denote by $a^{A}$ the conjugacy class in $A$ containing $a$; our notation for unipotent classes is taken from [11], which extends the Bala-Carter labelling to cover all characteristics. If $A$ is an algebraic group, $R_{u}(A)$ denotes its unipotent radical, $L(A)$ denotes its Lie algebra, and $V_{A}\left(\lambda_{i}\right)$ denotes the restricted irreducible $A$-module with high weight equal to the $i$ th fundamental weight. Write $U_{d}$ to denote a connected $d$-dimensional 
unipotent group, $T_{d}$ to denote a $d$-dimensional torus, and $Z_{d}$ to denote the cyclic group of order $d$.

We conclude this subsection by observing that the proof of [4, Proposition 5.1.1] generalizes to the situation here to show that the quotient map $G\langle\tau\rangle \rightarrow G\langle\tau\rangle / Z(G)$ restricts to a bijective morphism between the varieties of outer unipotent elements of the two groups, which induces a bijection between the outer unipotent classes; moreover $C_{Z(G)}(\tau)=1$, which means that if $v \in G \tau$ is an outer unipotent element then the quotient map induces an isomorphism between $C_{G}(v)$ and $C_{G / Z(G)}(v Z(G))$. It follows that results obtained in $G$ on outer unipotent classes and corresponding centralizers apply equally to any group isogenous to $G$ (provided it admits $\tau$ in the case $(G, p)=\left(D_{4}, 3\right)$ ); it therefore suffices to treat one group in each isogeny class.

\subsection{The number of outer unipotent elements}

Assume $(G, p)$ is any of the possibilities listed at the start of subsection 1.1. Recall that Steinberg proved that the number of unipotent elements in $G_{\gamma}$ (for any connected reductive group $G$ and Frobenius morphism $\gamma$ ) is equal to $\left(\left|G_{\gamma}\right|_{p}\right)^{2}$ (see [4, Theorem 6.6.1]). The following result extends this formula to the situation treated here.

Theorem 1.1 Assume $(G, p)=\left(A_{l}, 2\right),\left(D_{l}, 2\right),\left(E_{6}, 2\right)$ or $\left(D_{4}, 3\right)$. The number of p-elements in the coset $G_{\gamma} \tau$ is equal to

$$
\frac{\left|G_{\gamma}\right|}{\left|G_{\gamma, \tau}\right|} \cdot\left(\left|G_{\gamma, \tau}\right|_{p}\right)^{2}
$$

Our proof of Theorem 1.1 shows that the conclusion also holds if we replace $G_{\gamma}$ by $O^{p^{\prime}}\left(G_{\gamma}\right)$, and so holds in particular when this is a finite simple group.

\subsection{The $A_{l}$ case}

Assume $(G, p)=\left(A_{l}, 2\right)$; write $n=l+1$ and take $G$ to be simply connected, so that $G=S L_{n}(K)$. We can regard $G\langle\tau\rangle$ as a subgroup of $H=S p_{2 n}(K)$. Namely, if $V=V_{2 n}(K)$ is the natural module for $H$, then we can take $G$ to stabilize a pair $E, F$ of maximal totally singular subspaces of $V$, and $\tau$ to be an involution in $H$ interchanging $E$ and $F$.

Let $v \in G \tau$ be a unipotent element, and let $u=v^{2} \in G$. If we have $E \downarrow u=\sum J_{r_{i}}$ (a sum of Jordan blocks) with $\sum r_{i}=n$, then since $\tau$ interchanges $E$ and $F$ we have $V \downarrow v=\sum J_{2 r_{i}}$. By [11, Lemma 6.2], $V \downarrow v$ is a sum of indecomposables of the form $W\left(2 r_{i}\right.$ ) (a sum of two singular blocks of size $2 r_{i}$ ) and $V\left(2 r_{i}\right)$ (a single non-degenerate block of size $2 r_{i}$, appearing with multiplicity at most 2). Moreover, $V \downarrow u=\sum W\left(r_{i}\right)$ because $G$ fixes the pair $E, F$ of singular subspaces. From Lemma 4.1 below it follows that no summands $V\left(2 r_{i}\right)$ 
with $r_{i}$ even can be present in $V \downarrow v$; so we may write

$$
V \downarrow v=\sum W\left(2 r_{i}\right)^{a_{i}}+\sum_{s_{i} \text { odd }} V\left(2 s_{i}\right)^{c_{i}}
$$

(an orthogonal decomposition), where each $c_{i} \leq 2$. Rewrite this as

$$
V \downarrow v=\sum_{m_{i} \text { odd }} W\left(2 m_{i}\right)^{a_{i}}+\sum_{n_{i} \text { even }} W\left(2 n_{i}\right)^{b_{i}}+\sum_{k_{i} \text { odd }} V\left(2 k_{i}\right),
$$

where the $m_{i}$ and $n_{i}$ are distinct, and the $k_{i}$ are in non-increasing order and occur with multiplicity at most 2 . The corresponding element $u=v^{2} \in G$ acts on the $n$-dimensional space $E$ as

$$
E \downarrow u=\sum_{m_{i} \text { odd }} J_{m_{i}}{ }^{2 a_{i}}+\sum_{n_{i} \text { even }} J_{n_{i}}{ }^{2 b_{i}}+\sum_{k_{i} \text { odd }} J_{k_{i}} .
$$

In particular, even block sizes in $E \downarrow u$ occur with even multiplicity.

It follows from [16, pp.21-24] (see Lemma 4.2 below) that any two unipotent elements $v \in G \tau$ which are $H$-conjugate are also $G$-conjugate. Hence the above decomposition $V \downarrow v$ in (1) determines the $G$-class of $v$ uniquely. Moreover, the right hand side of (2) is also the Jordan decomposition of a unipotent element $u_{0}$ of the orthogonal group $O_{n}(\mathbb{C})$, and $[16, \mathrm{p} .22]$ gives

$$
\operatorname{dim} C_{G}(v)=\operatorname{dim} C_{O_{n}(\mathbb{C})}\left(u_{0}\right)+\sum_{\epsilon\left(m_{i}\right)=0} 2 a_{i},
$$

where $\epsilon\left(m_{i}\right)=0$ if there is no $k_{j}$ equal to $m_{i}$, and 1 otherwise. The formula for $\operatorname{dim} C_{O_{n}(\mathbb{C})}\left(u_{0}\right)$ can be found in [11, Proposition 3.7]. Namely, rewriting the right hand side of (2) as $\bigoplus_{i} J_{i}^{r_{i}}$ we have

$$
\operatorname{dim} C_{O_{n}(\mathbb{C})}\left(u_{0}\right)=\frac{1}{2} \sum_{i} i r_{i}^{2}+\sum_{i<j} i r_{i} r_{j}-\frac{1}{2} \sum_{i \text { odd }} r_{i} .
$$

Here is our main result about outer unipotent classes in $G \tau$. Parts (i) and (ii) summarize the above discussion; part (iii) determines the reductive parts of centralizers, and is our new contribution.

Theorem 1.2 Assume $(G, p)=\left(S L_{n}(K), 2\right)$.

(i) Each decomposition (1) represents a unique G-class of unipotent elements in $G \tau$.

(ii) For $v$ as in (1), the dimension of $C_{G}(v)$ is given by (3) and (4).

(iii) The reductive part of $C=C_{G}(v)$ is

$$
C / R_{u}(C)=\prod_{m_{i} \text { odd }} S p_{2 a_{i}}(K) \times \prod_{n_{i} \text { even }} I_{2 b_{i}}(K) \times Z_{2}{ }^{t+\delta},
$$


where

$$
I_{2 b_{i}}(K)= \begin{cases}S p_{2 b_{i}}(K) & \text { if } \exists k_{j}=n_{i} \pm 1, \\ O_{2 b_{i}}(K) & \text { otherwise, }\end{cases}
$$

$t$ is the number of $j$ such that $k_{j}>k_{j+1}+2>3$, and $\delta$ is 1 if there exists $k_{j} \geq 3$ and is 0 otherwise.

We give some tables illustrating this result for $n \leq 8$ in section 6 .

Theorem 1.2 yields results for the finite groups $G_{\gamma}\langle\tau\rangle=S L_{n}^{\epsilon}(q) .2$ just as in [11, Theorem 7.3], where $S L_{n}^{\epsilon}(q)=S L_{n}(q)$ or $S U_{n}(q)$ according as $\epsilon=1$ or $\epsilon=-1$.

Theorem 1.3 Assume $(G, p)=\left(S L_{n}(K), 2\right)$ and let $\gamma$ be a Frobenius morphism of $G$ with $G_{\gamma}=S L_{n}^{\epsilon}(q)$. Let $v \in G \tau$ be unipotent and write $C=C_{G}(v)$. Then $v^{G} \cap G_{\gamma} \tau$ splits into $2^{s+t+\delta} G_{\gamma}$-classes, where $t$ and $\delta$ are as in Theorem 1.2 and $s$ is the number of $O_{2 b_{i}}(K)$ factors in $C / R_{u}(C)$. For $x$ in such a $G_{\gamma}$-class, $C_{G_{\gamma}}(x)$ is an extension of $D_{\gamma}$ by $R$, where $D=R_{u}\left(C_{G}(u)\right)\left(\right.$ so $\left|D_{\gamma}\right|=q^{\operatorname{dim} D}$ ) and

$$
R=\prod_{m_{i} \text { odd }} S p_{2 a_{i}}(q) \times \prod_{n_{i} \text { even }} I_{2 b_{i}}(q) \times Z_{2}{ }^{t+\delta},
$$

where

$$
I_{2 b_{i}}(q)= \begin{cases}S p_{2 b_{i}}(q) & \text { if } \exists k_{j}=n_{i} \pm 1 \\ O_{2 b_{i}}^{\epsilon_{i}}(q) & \text { otherwise. }\end{cases}
$$

Moreover, all $2^{s}$ possibilities for $R$ occur equally often among the $2^{s+t+\delta} G_{\gamma^{-}}$ classes.

Theorem 1.3 will be deduced from Theorem 1.2 in subsection 4.5 .

\subsection{The $E_{6}$ and $D_{4}$ cases}

Assume $(G, p)=\left(E_{6}, 2\right)$ or $\left(D_{4}, 3\right)$. Our main results here are as follows.

Theorem 1.4 Assume $(G, p)=\left(E_{6}, 2\right)$. Then the coset $G \tau$ contains 17 conjugacy classes of unipotent elements under the action of $G$, with representatives $v_{1}, \ldots, v_{17}$ and centralizers given in Table $\%$. The conjugacy classes and centralizer orders of outer 2-elements in the coset $G_{\gamma} \tau$ are given in Table 9.

Theorem 1.5 Assume $(G, p)=\left(D_{4}, 3\right)$. Then the coset $G \tau$ contains 5 conjugacy classes of unipotent elements under the action of $G$, with representatives $v_{1}, \ldots, v_{5}$ and centralizers given in Table 8. The conjugacy classes and centralizer orders of outer 3-elements in the coset $G_{\gamma} \tau$ are given in Table 10.

The tables referred to in Theorems 1.4 and 1.5 can be found in section 6 . Note that in the third column of Table 7 or 8 we list the $E_{7}$-class or $F_{4}$-class of each representative $v_{i}$. 
Layout The rest of this paper is divided into five further sections. In section 2 we prove Theorem 1.1. In section 3 we establish some preliminary results, including the result of Fulman-Guralnick mentioned above. In section 4 we prove Theorems 1.2 and 1.3, and in section 5 we prove Theorems 1.4 and 1.5. Finally section 6 contains the tables for $E_{6}$ and $D_{4}$ referred to in Theorems 1.4 and 1.5, and also tables illustrating Theorem 1.2 for $G=S L_{n}(K)$ with $n \leq 8$. At the end there is an appendix giving extra detailed information used in the proof of Theorems 1.4 and 1.5.

Acknowledgements The research of the second and third authors was supported by an EPSRC grant. We also thank Gunter Malle who suggested a possible approach to Theorem 1.1 using a result of Digne-Michel in [5, 3.18].

\section{Proof of Theorem 1.1}

In this section we assume $(G, p)$ is any of the possibilities listed at the start of subsection 1.1, and that $\tau$ and $\gamma$ are as stated therein; we shall prove Theorem 1.1. The proof we shall give is an elaboration of Steinberg's original proof of the number of unipotent elements of a simple group of Lie type, which was based on the values of the Steinberg character. Here we use information established in $[5,3.18]$ on values of an extension of the Steinberg character.

First assume that $G$ is simply connected and $p=2$, so that $G_{\gamma}$ is $S L_{n}^{\epsilon}(q)$, $\Omega_{n}^{\epsilon}(q)$ or $E_{6}^{\epsilon}(q)$, with $q$ even. Abusing notation slightly we identify $\tau$ with its restriction to $G_{\gamma}$, and form the semidirect product $\hat{G}_{\gamma}:=G_{\gamma}\langle\tau\rangle$. Define an element $x \in G \tau$ to be quasi-semisimple if $x$ normalizes a Borel subgroup of $G$ and a maximal torus therein. By [5, 3.18], the Steinberg character of $G_{\gamma}$ can be extended to $\hat{G}_{\gamma}$, and the extension $\chi$ satisfies the following condition for elements $x \in G_{\gamma} \tau$ :

$$
\chi(x)= \begin{cases} \pm\left|C_{G_{\gamma}}(x)\right|_{2} & \text { if } x \text { is quasi-semisimple } \\ 0 & \text { otherwise. }\end{cases}
$$

In the following result, by a finite group of Lie type we mean a quotient by a central subgroup of the fixed point group under a Frobenius morphism of a simple algebraic group of simply connected type. For a group $X$, denote by $X^{(2)}$ the set of 2-elements in $X$.

Lemma 2.1 Let $A \times B$ be the direct product of two isomorphic finite groups of Lie type $A, B$ in characteristic 2 , and let $\tau$ be an involutory automorphism of $A \times B$ interchanging the two factors. Form the semidirect product $(A \times$ $B)\langle\tau\rangle$. Then the number of unipotent elements in the coset $(A \times B) \tau$ is equal to $|A B| /\left|C_{A B}(\tau)\right| \cdot\left|A^{(2)}\right|$.

Proof Identify $A$ and $B$, and write $\tau=t(\alpha, \beta)$ where $t$ is the map $\left(a, a^{\prime}\right) \mapsto$ $\left(a^{\prime}, a\right)$ (for $a, a^{\prime} \in A$ ) and $\alpha, \beta \in \operatorname{Aut}(A)$. As $\tau$ is an involution we have $\beta=\alpha^{-1}$. 
Suppose $(x, y) \tau$ is unipotent, where $x, y \in A$. Then $((x, y) \tau)^{2}=\left(x y^{\alpha}, y x^{\beta}\right)$ is unipotent, so $x y^{\alpha}, y x^{\beta}$ are unipotent elements of $A$. Conversely, given any $y \in A$ and any unipotent element $u \in A$, let $x=u\left(y^{\alpha}\right)^{-1}$; then $x y^{\alpha}=u$ is unipotent, as is $y x^{\beta}=y u^{\beta}\left(\left(y^{\alpha}\right)^{-1}\right)^{\beta}=y u^{\beta} y^{-1}$. Hence the number of unipotent elements of the form $(x, y) \tau$ is $|A| \cdot\left|A^{(2)}\right|=|A B| /\left|C_{A B}(\tau)\right| \cdot\left|A^{(2)}\right|$, as required.

Lemma 2.2 Let $\hat{G}_{\gamma}$ be as above, and let $s \in G_{\gamma}$ be a semisimple element such that $C_{\hat{G}_{\gamma}}(s)$ contains a unipotent element in the coset $G_{\gamma} \tau$. Then $C_{\hat{G}_{\gamma}}(s)=$ $D R\langle t\rangle$, where $D$ is a commuting product of groups of Lie type in characteristic $2, R$ is a maximal torus of $G_{\gamma}$ normalizing $D$, and $t$ is a quasi-semisimple involution normalizing both $R$ and a Borel subgroup of $D R$.

Proof As $G$ is simply connected, $C_{G}(s)$ is connected, so that $C_{G}(s)=\bar{D} \bar{R}$, where $\bar{D}$ is a product of simple algebraic groups and $\bar{R}$ is a maximal torus. We can take $\gamma$ to normalize $\bar{R}$ and a Borel subgroup $\bar{B}$ of $\bar{D}$. Then $C_{G_{\gamma}}(s)=$ $D R$, where $D=O^{2^{\prime}}\left(\bar{D}_{\gamma}\right)$ and $R=\bar{R}_{\gamma}$ (see $\left.[15,2.12]\right)$. Note that $D$ is a commuting product of groups of Lie type. We are assuming that $C_{\hat{G}_{\gamma}}(s)$ contains a unipotent element of $G_{\gamma} \tau$. Therefore $C_{\hat{G}_{\gamma}}(s)=D R\langle x\rangle$, where $x^{2} \in D R$. Then $N_{C_{\hat{G}_{\gamma}}(s)}\left(\bar{B}_{\gamma}\right) \cap N_{C_{\hat{G}_{\gamma}}(s)}(R)=R\langle t\rangle$ where $t$ is as in the statement of the lemma. Also $C_{\hat{G}_{\gamma}}(s)=D R\langle x\rangle=D R\langle t\rangle$.

Lemma 2.3 Let $s, t$ be as in Lemma 2.2, and write $C=C_{G_{\gamma}}(s)=D R$. Then

$$
\left|C_{C}(t)\right|=\left|C_{D}(t)\right| \cdot\left|C_{R / R \cap D}(t)\right|
$$

Proof As $C=D R$, we have $R / R \cap D \cong C / D$ by an isomorphism commuting with $t$. Therefore, $\left|C_{C / D}(t)\right|=\left|C_{R / R \cap D}(t)\right|$. Also $R$ is abelian of odd order, so that $R=C_{R}(t) \times[R, t]$ and $R \cap D=C_{R \cap D}(t) \times[R \cap D, t]$. It follows that $C_{R / R \cap D}(t) \cong C_{R}(t) / C_{R \cap D}(t)$. In particular, $C_{C}(t)$ covers $C_{C / D}(t)$. Hence $\left|C_{R / R \cap D}(t)\right|=\left|C_{C / D}(t)\right|=\left|C_{C}(t)\right| /\left|C_{D}(t)\right|$, as required.

Write $Z=Z\left(G_{\gamma}\right)$. In the next two lemmas we count the elements of $\hat{G}_{\gamma}$ in two different ways.

Lemma 2.4 We have $\left|\hat{G}_{\gamma}\right|=\Sigma_{1}+\Sigma_{2}+\Sigma_{3}$, where

$$
\begin{aligned}
\Sigma_{1} & =\sum_{s}\left|C_{\hat{G}_{\gamma}}(s)^{(2)}\right|, \text { sum over } s \in G_{\gamma} \backslash Z \text { semisimple, } \\
\Sigma_{2} & =|Z| \cdot\left|G_{\gamma}{ }^{(2)}\right| \\
\Sigma_{3} & =\left|\hat{G}_{\gamma}{ }^{(2)}\right|-\left|G_{\gamma}{ }^{(2)}\right| .
\end{aligned}
$$

Proof Observe that $\Sigma_{1}$ is the number of elements in $\hat{G}_{\gamma}$ with semisimple part $s \notin Z, \Sigma_{2}$ is the number of elements in $G_{\gamma}$ with semisimple part in $Z$, and $\Sigma_{3}$ is the number of remaining elements in $\hat{G}_{\gamma}\left(\right.$ since $\left.C_{Z}(\tau)=1\right)$. 
Lemma 2.5 We have $\left|\hat{G}_{\gamma}\right|=\Delta_{a}+\Delta_{b}+\Delta_{c}+\Delta_{d}$, where

$$
\begin{aligned}
\Delta_{a}= & \sum_{s}\left(\left|C_{G_{\gamma}}(s)\right|_{2}\right)^{2}, \text { sum over } s \in G_{\gamma} \backslash Z \text { semisimple, } \\
\Delta_{b}= & \sum_{s x}\left(\left|C_{G_{\gamma}}(s, x)\right|_{2}\right)^{2}, \text { sum over } s x \in \hat{G}_{\gamma} \text { quasi-semisimple with } \\
& \text { semisimple part } s \in G_{\gamma} \backslash Z, \\
\Delta_{c}= & |Z|\left(\left|G_{\gamma}\right|_{2}\right)^{2}, \\
\Delta_{d}= & \sum_{x}\left(\left|C_{G_{\gamma}}(x)\right|_{2}\right)^{2}, \text { sum over } x \in \hat{G}_{\gamma} \text { quasi-semisimple of order } 2 .
\end{aligned}
$$

Proof Let $\chi$ be the Steinberg character of $\hat{G}_{\gamma}$. Then $(\chi, \chi)=1$ implies that $\left|\hat{G}_{\gamma}\right|=\sum_{g \in \hat{G}_{\gamma}} \chi(g)^{2}$, and we have simply broken up the sum into the four parts $\Delta_{a}, \ldots, \Delta_{d}$, where $\Delta_{a}$ and $\Delta_{c}$ arise from applying the usual Steinberg character to elements of $G_{\gamma}$, while $\Delta_{b}$ and $\Delta_{d}$ arise from applying the extended character to elements in $G_{\gamma} \tau$.

From the previous two lemmas we have

$$
\Sigma_{1}+\Sigma_{2}+\Sigma_{3}=\Delta_{a}+\Delta_{b}+\Delta_{c}+\Delta_{d}
$$

Lemma 2.6 We have $\Sigma_{1}=\Delta_{a}+\Delta_{b}$.

Proof Obviously $\Sigma_{1}=\Sigma_{1}{ }^{\prime}+\Sigma_{1}{ }^{\prime \prime}$ where

$$
\Sigma_{1}^{\prime}=\sum_{s}\left|C_{G_{\gamma}}(s)^{(2)}\right|, \quad \Sigma_{1}^{\prime \prime}=\sum_{s}\left|C_{\hat{G}_{\gamma}}(s)^{(2)}\right|-\left|C_{G_{\gamma}}(s)^{(2)}\right| .
$$

By Steinberg's formula [4, Theorem 6.6.1] for the number of unipotent elements in each group $C_{G_{\gamma}}(s)$, we have $\Sigma_{1}{ }^{\prime}=\Delta_{a}$; so we need to show that $\Sigma_{1}{ }^{\prime \prime}=\Delta_{b}$.

Let $s \in G_{\gamma} \backslash Z$ be a fixed semisimple element and write $C=C_{G_{\gamma}}(s)$. As in Lemma 2.2 we have $C_{\hat{G}_{\gamma}}(s)=C\langle t\rangle=D R\langle t\rangle$. An outer unipotent element in $C\langle t\rangle$ projects to an involution in $C\langle t\rangle / D$, and these have the form Drt with $r t \in R t$ an involution. Such involutions $r t$ are all $R$-conjugate to $t$, so the total number of outer unipotent elements in the coset $C t$ is the number in $D t$ times the number of cosets Drt.

Now $t$ acts on the set of Lie type factors of $D$, with each factor being either normalized or interchanged with an isomorphic factor. Let $\tilde{D}$ be the universal cover of $D$, so that $\tilde{D}$ is a direct product of Lie type factors and we can pull back the action of $t$. Then using induction together with Lemma 2.1, we see that the number of unipotent elements in the coset $\tilde{D} t$ is equal to

$$
\frac{|\tilde{D}|}{\left|C_{\tilde{D}}(t)\right|}\left(\left|C_{\tilde{D}}(t)\right|_{2}\right)^{2}
$$

Write $D=\tilde{D} / J$, where $J$ is abelian of odd order, and let $J^{-}$be the set of elements in $J$ inverted by $t$. Then each unipotent element in $D t$ pulls back 
to $\left|J^{-}\right|$unipotent elements in $\tilde{D} t$, all of which are conjugate under $J^{-}$. As $\left(\left|C_{\tilde{D}}(t)\right|_{2}\right)^{2}=\left(\left|C_{D}(t)\right|_{2}\right)^{2}$, we see that the number of unipotent elements in the coset $D t$ is equal to

$$
\frac{|D|}{\left|C_{D}(t)\right|}\left(\left|C_{D}(t)\right|_{2}\right)^{2}
$$

The number of cosets Drt with $r t$ an involution is $|R / R \cap D| /\left|C_{R / R \cap D}(t)\right|=$ $|C / D| /\left|C_{R / R \cap D}(t)\right|$. Hence by Lemma 2.3 and the above equation, the number of unipotent elements in the coset $C t$ equals $\left(|C| /\left|C_{C}(t)\right|\right)\left(\left|C_{D}(t)\right|_{2}\right)^{2}=$ $\left(|C| /\left|C_{C}(t)\right|\right)\left(\left|C_{G_{\gamma}}(s, t)\right|_{2}\right)^{2}$. The quasi-semisimple elements $s x \in \hat{G}_{\gamma}$ having semisimple part $s$ and $x$ of order 2 are all conjugate to $s t$. So summing over $s$ we obtain $\Sigma_{1}{ }^{\prime \prime}=\Delta_{b}$, as required.

We now complete the proof of Theorem 1.1 for the cases where $G$ is simply connected and $p=2$. By Steinberg's formula we have $\Sigma_{2}=\Delta_{c}$, and hence by (5) and Lemma 2.6, $\Sigma_{3}=\Delta_{d}$. All the quasi-semisimple involutions $x \in \hat{G}_{\gamma}$ are conjugate to $\tau$, so $\Delta_{d}=\left|G_{\gamma}: G_{\gamma, \tau}\right| \cdot\left(\left|G_{\gamma, \tau}\right|_{2}\right)^{2}$. Since $\Sigma_{3}$ is the number of unipotent elements in $G_{\gamma} \tau$, the result follows.

Next we relax the assumption that $G$ is simply connected. Let $\tilde{G}$ be the simply connected cover of $G$. We can consider $\gamma$ and $\tau$ acting on $\tilde{G}$. There is a natural surjection $\tilde{G}_{\gamma} \rightarrow O^{p^{\prime}}\left(G_{\gamma}\right)$. Let $X=\tilde{G}_{\gamma} / Z\left(\tilde{G}_{\gamma}\right) \cong O^{p^{\prime}}\left(G_{\gamma}\right) / Z\left(O^{p^{\prime}}\left(G_{\gamma}\right)\right)$. Using [14, 2.3 and 2.4] we see that $\left|Z\left(\tilde{G}_{\gamma}\right)\right|=\left|Z\left(G_{\gamma}\right)\right| \cdot\left(\left|G_{\gamma}\right| /\left|O^{p^{\prime}}\left(G_{\gamma}\right)\right|\right)$. Moreover, the outer unipotent elements in $G_{\gamma} \tau$ invert both $Z\left(G_{\gamma}\right)$ and $G_{\gamma} / O^{p^{\prime}}\left(G_{\gamma}\right)$. The above argument with $\tilde{D}$ and $D$ shows that both the number of outer unipotent elements and the formula in Theorem 1.1 are independent of the form of $G$. Indeed, in either case the number of outer unipotent elements equals $\left|Z\left(\tilde{G}_{\gamma}\right)\right|$ times the number of outer unipotent elements in $X \tau$. We note that the theorem also holds if we replace $G_{\gamma}$ by $O^{p^{\prime}}\left(G_{\gamma}\right)$.

It remains to handle the $p=3$ case of Theorem 1.1. Starting with $G=D_{4}$ simply connected this can be achieved by arguing as above with obvious changes along the way, and this is left to the reader. (We note that the only place requiring an analogue of Lemma 2.1 is where $\tau$ transitively permutes the factors in the direct product of three copies of $S L_{2}$; so $\tilde{D}=D$ in this situation.) The argument of the above paragraph then gives the result when $G$ is an adjoint group. Alternatively, the result can be established easily from the information given in Table 10 in section 6 .

\section{Preliminary lemmas}

In this section we assume $(G, p)=\left(A_{l}, 2\right),\left(E_{6}, 2\right)$ or $\left(D_{4}, 3\right)$. We shall first prove a special case of a result of Fulman and Guralnick [6, Lemma 2.2], and then establish some basic lemmas which combine this result with information from [11]. In particular we count the number of $G_{\gamma}$-classes of $p$-elements in $G_{\gamma} \tau$. For each $p$-element in $G \tau$ we then introduce a parabolic subgroup of $G$ which will play an important role in the determination of the $G$-centralizer. 
Lemma 3.1 Let $X$ be a finite group with normal subgroup $Y$ of index $p$, and let $\tau \in X \backslash Y$ have order $p$. Then the number of $X$-orbits on p-elements in $Y \tau$ equals the number of $\tau$-stable $Y$-orbits on p-elements in $Y$.

Proof Observe first that if $x \in X$ then

$$
x^{Y} \text { is } \tau \text {-stable } \Longleftrightarrow x^{Y}=x^{X} \Longleftrightarrow C_{X}(x) \cap Y \tau \neq \emptyset .
$$

Write

$$
P_{1}=\{p \text {-elements in } Y \tau\}, \quad P_{2}=\{p \text {-elements in } Y \text { with } \tau \text {-stable } Y \text {-orbit }\} .
$$

Then for $i=1,2$ the $X$-orbits on $P_{i}$ are the same as the $Y$-orbits on $P_{i}$; write $n_{i}$ for this common number of orbits. We seek to show that $n_{1}=n_{2}$.

Take $i \in\{1,2\}$; given $x \in X$ write $F_{i}(x)=\left|C_{X}(x) \cap P_{i}\right|$. For $x \in X \backslash Y$, the generators of $\langle x\rangle$ are distributed in equal numbers among the cosets $Y \tau^{i}$ for $i \neq 0$, so we have both

$$
n_{i}=\frac{1}{|X|} \sum_{x \in X} F_{i}(x)=\frac{1}{|X|} \sum_{y \in Y} \sum_{j=0}^{p-1} F_{i}\left(y \tau^{j}\right)=\frac{1}{|X|} \sum_{y \in Y}\left(F_{i}(y)+(p-1) F_{i}(y \tau)\right)
$$

and

$$
n_{i}=\frac{1}{|Y|} \sum_{y \in Y} F_{i}(y)
$$

whence

$$
n_{i}=\frac{1}{|Y|} \sum_{y \in Y} F_{i}(y \tau)
$$

Now take $x=y \tau \in Y \tau$; choose $k \equiv 1(\bmod p)$ such that $z=x^{k}$ is a $p$ element, so certainly $z \in C_{X}(x) \cap Y \tau$. Thus the map $g \mapsto z g$ is a bijection $C_{Y}(x) \rightarrow C_{X}(x) \cap Y \tau$; and $g \in C_{Y}(x)$ is a $p$-element if and only if $z g \in$ $C_{X}(x) \cap Y \tau$ is a $p$-element. Since any element of $C_{Y}(x)$ has a $\tau$-stable $Y$-orbit by the initial observation, we thus have a bijection $C_{X}(x) \cap P_{2} \rightarrow C_{X}(x) \cap P_{1}$, whence $F_{2}(x)=F_{1}(x)$. It follows that $n_{2}=n_{1}$ as required.

Our next two results show (as is done in $[6,2.6]$ ) that the finiteness of the number of outer unipotent classes in the coset $G \tau$ follows from combining Lemma 3.1 with the finiteness of the number of unipotent classes in $G$.

Let $p(n)$ denote the number of partitions of $n$.

Lemma 3.2 Assume $G$ is adjoint.

(i) If $(G, p)=\left(A_{l}, 2\right)$, then $G_{\gamma}$ has $p(l+1)$ conjugacy classes of 2-elements, each of which is $\tau$-stable.

(ii) If $(G, p)=\left(E_{6}, 2\right)$, then $G_{\gamma}$ has 28 conjugacy classes of 2-elements, each of which is $\tau$-stable. 
(iii) If $(G, p)=\left(D_{4}, 3\right)$, then $G_{\gamma}$ has 7 conjugacy classes of 3 -elements which are $\tau$-stable.

Proof (i) We know that $G$ has $p(l+1)$ classes of unipotent elements and [11, Theorem 7.1] shows that the same holds for the finite linear and unitary groups.

(ii) The first assertion follows from [11, Corollary 17.7]. For the second assertion we make use of the explicit information on centralizers of unipotent classes of $G(q)$ presented in [11, Table 22.2.3]. Let $u$ be a unipotent element of $G_{\gamma}$, and write $u^{\prime}=u^{\tau}$. We claim that $u^{\prime}=u^{g}$ for some element $g \in G_{\gamma}$.

Set $C_{u}=C_{G_{\gamma}}(u)$ and $C_{u^{\prime}}=C_{G_{\gamma}}\left(u^{\prime}\right)$. By comparing first the sizes of $O_{2}\left(C_{u}\right)$ and $O_{2}\left(C_{u^{\prime}}\right)$, and then the orders of $C_{u}$ and $C_{u^{\prime}}$, we see that $u$ and $u^{\prime}$ are $G_{\gamma}$-conjugate except possibly for the cases where $u$ has $G$-class $D_{4}, D_{5}$, $E_{6}$ or $E_{6}\left(a_{3}\right)$. In each of these cases the $G$-class splits into two $G_{\gamma}$-classes of equal size; so the only question is whether $\tau$ fixes each of the two classes or interchanges them. Now $G_{\gamma, \tau}=F_{4}(q)$ contains unipotent elements of type $B_{3}$, $B_{4}, F_{4}$, and these elements lie in the $G$-classes $D_{4}, D_{5}, E_{6}$ respectively; so $\tau$ must fix each of these $G$-classes. Also if $u$ has type $F_{4}\left(a_{2}\right)$ in $F_{4}$ then we see from [11, Table 22.1.4] that $u$ lies in the $G$-class $E_{6}\left(a_{3}\right)$; moreover, a representative for the class of $u$ can be taken over the prime field (see the discussion at the start of [11, Chapter 18]). So here too $\tau$ fixes each of the classes, establishing the claim. This completes the proof of (ii).

(iii) Here we begin at the level of the orthogonal group $\mathrm{SO}_{8}(K)$, whose unipotent classes are tabulated in [11, Table 8.5a]. Those with distinguished normal form $W(1)^{4}, W(2)+W(1)^{2}, W(3)+W(1), W(2)+V(3)+V(1), V(5)+V(3)$, $V(7)+V(1)$ have Bala-Carter labels $1, A_{1}, A_{2}, D_{2} A_{1}=A_{1}{ }^{3}, D_{4}\left(a_{1}\right), D_{4}$ respectively, while those with distinguished normal form $W(2)^{2}$ (two classes), $W(1)^{2}+V(3)+V(1), W(4)$ (two classes), $W(1)+V(5)+V(1)$ have Bala-Carter labels $\left(A_{1}^{2}\right)^{\prime}$ or $\left(A_{1}^{2}\right)^{\prime \prime}, D_{2},\left(A_{3}\right)^{\prime}$ or $\left(A_{3}\right)^{\prime \prime}, D_{3}$ respectively. Now take the corresponding classes in $G$. In each of the first six cases the triality automorphism $\tau$ stabilizes a root subsystem of the relevant type, and hence the class of regular (or in one case subregular) unipotent elements in the corresponding subsystem subgroup; in the second six cases, however, $\tau$ cycles the first three and the second three classes. By [11, Theorem 3.1] the centralizer for the $A_{2}$ class has component group of order 2, while that for each of the other five $\tau$-stable classes is connected; descending to the finite group $G_{\gamma}$ we see that (iii) holds.

Lemma 3.3 (i) If $G$ is adjoint, then according as $(G, p)=\left(A_{l}, 2\right),\left(E_{6}, 2\right)$ or $\left(D_{4}, 3\right)$ there are $p(l+1), 28$ or $7 G_{\gamma}$-classes of p-elements in $G_{\gamma} \tau$.

(ii) There are finitely many $G$-classes of p-elements in $G \tau$ (for $G$ of arbitrary isogeny type, provided $G$ admits $\tau$ in the case $\left.(G, p)=\left(D_{4}, 3\right)\right)$.

Proof Part (i) follows from Lemmas 3.1 and 3.2. For (ii) we first observe that it suffices to assume $G$ is adjoint by the final paragraph of subsection 1.1; moreover we can apply [8, Proposition 1.1] to assume that $K$ is the algebraic 
closure of the prime field. We claim that the number of $G$-classes of $p$-elements in $G \tau$ is at most $k$, where $k=p(l+1), 28$ or 7 respectively (later we shall see that there are precisely 17 such classes if $G=E_{6}$ and 5 classes if $G=D_{4}$ ). If this claim were false, there would be at least $k+1$ such classes, and we could choose a sufficiently large power $q$ of $p$ such that each class had a representative in $G_{\gamma} \tau$, where $\gamma=\sigma$ for $\sigma$ the $q$-field morphism as in subsection 1.1; but this would contradict (i). Therefore the claim holds and (ii) is established.

Let $v$ be a $p$-element in $G \tau$, and write $u=v^{p} \in G$. We bring into play a parabolic subgroup of $G$ which contains $C_{G}(u)$ and for which a certain density statement holds. In order to do this we make use of a certain nilpotent element $e$ in the Lie algebra $L(G)$. For $G=A_{l}$ we can view $G$ as $S L_{l+1}(K)$ and let $e=u-1$. For $G=E_{6}$ we use representatives of the unipotent classes of $G$ given in [11]; we can take $u$ to be a product of root elements $u=\prod u_{\beta_{i}}$ for certain $\beta_{i} \in \Sigma$, and then $u$ and the corresponding nilpotent element $e=\sum e_{\beta_{i}}$ are linked (see [11, p.281 and Theorem 17.3]). For $G=D_{4}$, this is described in [11, Lemmas 2.15, 3.13].

As is explained in [11, p.4], there is a certain 1-dimensional torus $T$ in $G$ which acts by weight 2 on $\langle e\rangle$; by taking an appropriate simple system we may assume for each simple root $\beta$ the $T$-weight on $\left\langle e_{\beta}\right\rangle$ is 0,1 or 2 , and we write $\Delta$ for the corresponding labelled Dynkin diagram. The torus $T$ determines a parabolic subgroup $P=Q L$, where $L=C_{G}(T)$ and $Q=\prod X_{\beta}$ with the product taken over those roots $\beta \in \Sigma$ for which the $T$-weight on $\left\langle e_{\beta}\right\rangle$ is positive; thus the simple roots of $L$ are those with label 0 in $\Delta$. We write $Q_{\geq 2}$ (respectively $Q_{>2}$ ) for the subgroup $\prod X_{\beta}$ of $Q$, where the product is taken over those roots $\beta$ such that the $T$-weight on $\left\langle e_{\beta}\right\rangle$ is at least 2 (respectively greater than 2). Then for each root element $u_{\beta}$ appearing in the expression for $u$, the $T$-weight on $\left\langle e_{\beta}\right\rangle$ is 2 , so that $u \in Q_{\geq 2}$ (see $\left.[11,(18.1)]\right)$.

If $(G, p)=\left(E_{6}, 2\right)$, the correspondence between unipotent classes $u^{G}$ and labelled Dynkin diagrams $\Delta$ is given in [11, Table 22.1.3]. If instead $(G, p)=$ $\left(A_{l}, 2\right)$ or $\left(D_{4}, 3\right)$, the labelled Dynkin diagram $\Delta$ can be obtained from the action of $T$ on the natural module $W=V_{G}\left(\lambda_{1}\right)$ as follows. We may assume $T$ is contained in the maximal torus $T_{G}$ of $G$; then the $T_{G^{-} \text {-weights on }}$ $W$ determine the $T$-weights on the simple roots $\alpha_{i}$. In the $A_{l}$ case the $T_{G^{-}}$ weights on $W$ are $\lambda_{1}, \lambda_{1}-\alpha_{1}, \lambda_{1}-\alpha_{1}-\alpha_{2}, \ldots$; thus if for example $l=9$ and $u$ (and hence $e$ ) acts as $J_{5}+J_{3}+J_{2}$, the $T$-weights on the individual blocks are $(4,2,0,-2,-4),(2,0,-2),(1,-1)$, so that the $T$-weights on $W$ are $4,2^{2}, 1,0^{2},-1,-2^{2},-4$, and hence $\Delta$ is 201101102. In the $D_{4}$ case the $T_{G^{-}}$ weights on $W$ are $\lambda_{1}, \lambda_{1}-\alpha_{1}, \lambda_{1}-\alpha_{1}-\alpha_{2}, \lambda_{1}-\alpha_{1}-\alpha_{2}-\alpha_{3}, \lambda_{1}-\alpha_{1}-\alpha_{2}-$ $\alpha_{4}, \ldots$; thus if for example $u$ (and hence $e$ ) has distinguished normal form $W(2)+V(3)+V(1)$ and so acts as $J_{3}+J_{2}{ }^{2}+J_{1}$, the $T$-weights on $W$ are $2,1^{2}, 0^{2},-1^{2},-2$, and hence $\Delta$ is $10 \frac{1}{1}$.

The following lemma follows from [11, Theorem 1] for $G=A_{l}, D_{4}$ and [11, Theorems 17.4, 17.5] for $G=E_{6}$. 
Lemma $3.4 \quad$ (i) $C_{G}(u) \leq P$;

(ii) $u^{P}$ is dense in $Q_{\geq 2}$;

(iii) $u Q_{>2}$ is fused under the action of $Q$.

Our final result in this section shows that if the parabolic subgroup $P$ is $\tau$-stable then it is stabilized by $v$.

Lemma 3.5 Assume that $P=P^{\tau}$. With $v$, $u$ and $P$ as above, we have $P=P^{v}$.

Proof We have $u \in Q_{\geq 2}<P$, and $u^{P}$ is dense in $Q_{\geq 2}$. By hypothesis $\tau$ normalizes $P$; thus $P^{v}=P^{g}$ for some element $g \in G$, and so $Q^{v}=Q^{g}$ and $\left(Q^{\prime}\right)^{v}=\left(Q^{\prime}\right)^{g}$. It follows from [2] that $Q_{\geq 2}=Q$ or $Q^{\prime}$, since the labels in the labelled Dynkin diagram $\Delta$ which determines $P$ are $0,1,2$. In either case $u \in\left(Q_{\geq 2}\right)^{v}=\left(Q_{\geq 2}\right)^{g}$ and this group is either $Q^{g}$ or $\left(Q^{\prime}\right)^{g}$.

Now $\operatorname{dim} C_{P}(u)=\operatorname{dim} C_{P^{v}}\left(u^{v}\right)=\operatorname{dim} C_{P^{v}}(u)=\operatorname{dim} C_{P^{g}}(u)$. A dimension comparison implies that $u^{P^{g}}$ is dense in $\left(Q_{\geq 2}\right)^{g}$. But $\left(u^{g}\right)^{P^{g}}$ is also dense in $\left(Q_{\geq 2}\right)^{g}$. Therefore, $\left(u^{g}\right)^{p^{g}}=u$ for some element $p \in P$, so that $p g \in C_{G}(u)=$ $C_{P}(u)$. Hence $g \in P$ and $P=P^{g}=P^{v}$ as required.

\section{Proof of Theorems 1.2 and 1.3}

In this section we assume $(G, p)=\left(A_{l}, 2\right)$. Most of this section is devoted to the proof of Theorem 1.2. Finally Theorem 1.3 is deduced in subsection 4.5.

\subsection{Preliminaries}

As in subsection 1.3 write $n=l+1$ and take $G$ to be simply connected, so that $G=S L_{n}(K)$; regard $G\langle\tau\rangle$ as a subgroup of $H=S p_{2 n}(K)$. Let $V=V_{2 n}(K)$ be the natural module for $H$ with symplectic form (, ), and write $V=E \oplus E^{\tau}$, where $G$ acts on each of the totally singular summands $E$ and $E^{\tau}$. First note that the fact that any unipotent element $u \in G$ acts on $E$ and $E^{\tau}$ forces $V \downarrow u$ to be a sum of summands of type $W(k)$. The following result justifies equation (1) of subsection 1.3.

Lemma 4.1 Let $v \in S p(V)$ be a unipotent element such that $V \downarrow v=V(2 k)$. Then

$$
V \downarrow v^{2}= \begin{cases}V(k)^{2} & \text { if } k \text { is even, } \\ W(k) & \text { if } k \text { is odd. }\end{cases}
$$

Proof Here $v$ acts on $V$ as follows. Take a basis $v_{2 k}, v_{2 k-1}, \ldots, v_{1}$ of $V(2 k)$, where the symplectic bilinear form is given by $\left(v_{i}, v_{j}\right)=1$ if $i+j=2 k+1$ and 
0 otherwise. Now define $v$ to act as

$$
\begin{aligned}
v_{k+i} & \mapsto v_{k+i}+v_{k+i-1}+\cdots+v_{k} \quad(1 \leq i \leq k), \\
v_{j} & \mapsto v_{j}+v_{j-1} \quad(2 \leq j \leq k), \\
v_{1} & \mapsto v_{1}
\end{aligned}
$$

Clearly $v^{2}$ has two Jordan blocks of size $k$. The question is whether these blocks are singular or non-degenerate (meaning that $v^{2}$ acts on $V$ as $W(k)$ or $V(k)^{2}$, respectively).

Assume first that $k$ is even. For $x \in V$ write $\left[x, v^{2}\right]=x+x v^{2},\left[x, v^{2}\right]^{(2)}=$ $\left[\left[x, v^{2}\right], v^{2}\right]$, and in general $\left[x, v^{2}\right]^{(i)}=\left[\ldots\left[\left[x, v^{2}\right], v^{2}\right], \ldots, v^{2}\right]$, the $i$-fold commutator. We compute inductively that for $i \leq \frac{k}{2}-1$, the commutator $\left[v_{2 k}, v^{2}\right]^{(i)}$ is of the form

$$
v_{2 k-2 i}+\sum_{j \text { even, } 2 k-2 i>j \geq k} \mu_{j} v_{j}+\sum_{l<k} \mu_{l} v_{l} .
$$

In particular,

$$
\left[v_{2 k}, v^{2}\right]^{\left(\frac{k}{2}-1\right)}=v_{k+2}+\mu_{k} v_{k}+\sum_{l<k} \mu_{l} v_{l}
$$

The next commutator is then of the form

$$
\left[v_{2 k}, v^{2}\right]^{\left(\frac{k}{2}\right)}=v_{k}+v_{k-1}+\sum_{l<k-1} \nu_{l} v_{l}
$$

As $\left[v_{i}, v^{2}\right]=v_{i-2}$ when $3 \leq i \leq k$, it follows that

$$
\left[v_{2 k}, v^{2}\right]^{(k-1)}=v_{2}+v_{1} .
$$

Since $\left(v_{2 k}, v_{2}+v_{1}\right) \neq 0$, it follows that the Jordan block for $v^{2}$ generated by $v_{2 k}$ is non-degenerate, hence is of type $V(k)$. Also $V(k)^{v}$ is another Jordan block for $v^{2}$ of the same type and orthogonal to $V(k)$; so $V \downarrow v^{2}=V(k)^{2}$ when $k$ is even.

Now assume $k$ is odd. In this case we compute inductively that for any $i$, the commutator $\left[v_{2 i}, v^{2}\right]$ involves only terms in $v_{j}$ for $j \leq 2 i-2$ even. Hence $\left[v_{2 k}, v^{2}\right]^{(k-1)}=v_{2}$, and so as $\left(v_{2 k}, v_{2}\right)=0$, the Jordan block for $v^{2}$ generated by $v_{2 k}$ is singular of size $k$. Applying $v$ to this block gives another singular block, and hence $V \downarrow v^{2}=W(k)$ when $k$ is odd. For use in the next lemma we note that $\left[v_{2 k},\left(\left[v_{2 k}, u\right]^{(k-1)}\right)^{v}\right]=\left[v_{2 k}, v_{2}^{v}\right]=\left[v_{2 k}, v_{2}+v_{1}\right]=1 \neq 0$.

In [16, p.21], Spaltenstein views elements of $G \tau$ as non-degenerate bilinear forms on $E$ and goes on to describe the conjugacy classes with respect to these forms. We can also view elements of $G \tau$ in this way as follows. Let $v$ and $u$ be as in equations (1) and (2) of subsection 1.3. Define a non-degenerate bilinear form $(,)_{v}$ on $E$ by $\left(e_{1}, e_{2}\right)_{v}=\left(e_{1}, e_{2}{ }^{v}\right)$. Let $\lambda$ be the partition of $n$ determined from the action of $u$ on $E$ and define a function $\epsilon: \mathbb{N} \rightarrow\{0,1, \omega\}$ which for all $i$ 
sends

$$
\begin{aligned}
n_{i} & \mapsto \omega, \\
k_{i} & \mapsto 1, \\
m_{i} & \mapsto \begin{cases}1 & \text { if } \exists k_{j}=m_{i}, \\
0 & \text { otherwise, }\end{cases}
\end{aligned}
$$

and sends all other positive integers to 0 . Thus $V \downarrow v$ determines a pair $(\lambda, \epsilon)$.

Lemma 4.2 Let $G\langle\tau\rangle=S L_{n}(K)\langle\tau\rangle<S p(V)=H$ as above. Then any two unipotent elements $v \in G \tau$ which are $H$-conjugate are also $G$-conjugate.

Proof According to [16, p.21], the $G$-classes of unipotent elements in $G \tau$ are in bijective correspondence with the set of pairs $(\lambda, \epsilon)$, where $\lambda$ is a partition of $n$ such that all even parts have even multiplicity, and $\epsilon: \mathbb{N} \rightarrow\{0,1, \omega\}$ is a function having various properties which are described on [16, p.22].

We wish to show that the function defined above agrees with the one given by Spaltenstein. The key facts which we must establish are that if $w$ is in a $W(2 m)$ summand of $V \downarrow v$, then $\left(w,\left([w, u]^{(m-1)}\right)^{v}\right)=0$, whereas in a $V(2 m)$ summand with $m$ odd there exists $w$ for which this does not hold. The latter was established at the end of the proof of Lemma 4.1.

Consider a summand of type $W(2 m)$. The action of $v$ is given on [11, p.92]. With a slight change of notation we see that $v$ has two Jordan blocks of size $2 m$ with bases $x_{1}, \ldots, x_{2 m}$ and $y_{1}, \ldots, y_{2 m}$, where $x_{1}$ and $y_{1}$ generate the blocks and $x_{2 m}$ and $y_{2 m}$ are fixed points. One shows by induction that for $i<2 m-1$ odd, we have $\left[x_{i}, u\right]=x_{i+2}+w_{i}$, where $w_{i}$ is a sum of terms $x_{j}$ for $j>i+2$ odd. It follows that the Jordan block for $u$ generated by $x_{1}$ has fixed space spanned by $x_{2 m-1}=\left[x_{1}, u\right]^{(m-1)}$. A similar conclusion holds for the block generated by $y_{1}$. Now $u$ has four Jordan blocks of size $m$ on $W(2 m)$, namely the two just described and their images under $v$. It is now straightforward to check that $\left(w,\left([w, u]^{(m-1)}\right)^{v}\right)=0$ for all $w \in W(2 m)$.

At this point we see that the function $\epsilon$ defined above agrees with the one defined in [16, p.22]. Therefore, the restriction $V \downarrow v$ uniquely determines $\epsilon$. The result follows.

\subsection{Some special cases}

The next few lemmas prove Theorem 1.2 in some key special cases. The first lemma settles two base cases which will be needed in a later inductive proof.

Lemma 4.3 If $V \downarrow v$ is either $W(4)+V(2)$ or $W(4)+V(6)$, then $C^{\circ} / R_{u}(C) \cong$ $S p_{2}$.

Proof By [11, Theorem 4.2] the full centralizer of $v$ in $S p(V)$ has its connected reductive part $S p_{2}$. Therefore $C^{\circ} / R_{u}(C)$ is contained in $S p_{2}$. Suppose that 
equality holds for the case $V \downarrow v=W(4)+V(6)$. Let $W=[V, v]^{(4)}$. Then $C$ acts on $W^{\perp} / W$ and $v$ acts on this space as $W(4)+V(2)$. So the equality for $W(4)+V(2)$ also holds.

Now consider $W(4)+V(6)$, where $G=S L_{7}(K)=A_{6}$ with fundamental roots $\alpha_{1}, \ldots, \alpha_{6}$. We use notation as in subsection 1.1 and set $v=\tau u_{111000} u_{011100}$. One checks that $u=v^{2}$ lies in the class $A_{2} A_{1}{ }^{2}$, and so has Jordan form $J_{3}+J_{2}{ }^{2}$. Therefore $v$ is necessarily in the correct class.

The labelled Dynkin diagram $\Delta$ corresponding to $u$ is 101101; thus the parabolic subgroup $P=Q L$ described prior to Lemma 3.4 has Levi factor of type $A_{1} A_{1}$. Using notation $x_{i j k}(d)=x_{\alpha_{i}+\alpha_{j}+\alpha_{k}}(d)$ and so forth one checks either by hand or by the computer technique described in the appendix that

$$
\begin{aligned}
C_{U}(v)=\{ & x_{2}\left(t_{1}^{2}\right) x_{5}\left(t_{1}^{2}\right) x_{34}\left(t_{2}\right) x_{12}\left(t_{1}\right) x_{56}\left(t_{1}\right) x_{23}\left(t_{1}\right) x_{45}\left(t_{1}\right) \times \\
& x_{2345}\left(t_{3}\right) x_{123}(\zeta) x_{456}(\zeta) x_{123456}\left(t_{4}\right) x_{234}\left(t_{5}\right) x_{345}\left(t_{5}\right) \times \\
& \left.x_{1234}\left(t_{6}\right) x_{3456}\left(t_{6}\right) x_{12345}\left(t_{7}\right) x_{23456}\left(t_{1}+t_{7}\right): \zeta \in \mathbb{F}_{2}, t_{i} \in K\right\} .
\end{aligned}
$$

Then $C_{U}(v) Q / Q=\left\{x_{2}(t) x_{5}(t): t \in K\right\} Q / Q$, a 1-dimensional unipotent subgroup of $P / Q$. In addition $s_{2} s_{5} x_{011100}(1) \in C_{P}(t)$, which implies that $C_{P}(t) Q / Q$ contains $S L_{2}=S p_{2}$, as required.

Lemma 4.4 Let $v \in S L_{n}\langle\tau\rangle\left\langle S p(V)\right.$ be such that $V \downarrow v=W(2 m)^{a}$ for some $m$, and let $C=C_{S L_{n}}(v)$. Then

$$
C / R_{u}(C)= \begin{cases}S p_{2 a} & \text { if } m \text { is odd }, \\ O_{2 a} & \text { if } m \text { is even. }\end{cases}
$$

Proof We can regard $V \downarrow v$ as $V(2 m) \otimes I_{2 a} \leq S p_{2 m} \otimes S p_{2 a}$, acting on a pair of singular spaces of the form $V(2 m) \otimes R$ and $V(2 m) \otimes S$. In $S p(V)$, the element $v$ is obviously centralized by the factor $S p_{2 a}$, and this is the full reductive part of the centralizer, by [11, Lemma 6.7].

If $m$ is odd, we easily obtain the result as follows. For then Lemma 4.1 implies that $u=v^{2}$ acts on $V$ as $\left(J_{m}+J_{m}{ }^{\prime}\right) \otimes I_{2 a}=W(m) \otimes I_{2 a}$, with $v$ interchanging the two singular blocks $J_{m}, J_{m}{ }^{\prime}$. Replace the above $S L_{n}$ by the $S p(V)$-conjugate which stabilizes each of the singular spaces $J_{m} \otimes I_{2 a}, J_{m}{ }^{\prime} \otimes I_{2 a}$. Then $v$ normalizes this $S L_{n}$ and since the $S p(V)$-class determines the $S L_{n}$-class, $v$ has the same centralizer in each $S L_{n}$. As $v$ clearly centralizes an $S p_{2 a}$ subgroup in the second $S L_{n}$, this gives the assertion when $m$ is odd.

Now suppose $m$ is even. Here Lemma 4.1 shows that $u=v^{2}$ acts on $V$ as $\left(J_{m}+J_{m}{ }^{\prime}\right) \otimes I_{2 a}=\left(V(m)+V(m)^{\prime}\right) \otimes I_{2 a}$, where this time the blocks $V(m), V(m)^{\prime}$ are non-degenerate and interchanged by $v$. Let $V_{2 a}$ be the natural module for the factor $S p_{2 a}$, and choose singular $a$-spaces $R, S$ in $V_{2 a}$ such that $V_{2 a}=R+S$. Define

$$
W=(V(m) \otimes R)+\left(V(m)^{\prime} \otimes S\right), \quad W^{\prime}=(V(m) \otimes S)+\left(V(m)^{\prime} \otimes R\right) .
$$


These are singular $2 a m$-spaces in $V$ interchanged by $v$. We take our group $S L_{n}\langle\tau\rangle=S L_{n}\langle v\rangle$ in the stabilizer in $S p(V)$ of the pair $\left\{W, W^{\prime}\right\}$. The centralizer $C_{S L_{n}}(v)$ clearly contains the subgroup $G L_{a} .2$ of $S p_{2 a}$ which fixes or interchanges the pair $R, S$. Hence $G L_{a} .2 \leq C / R_{u}(C) \leq S p_{2 a}$.

Next we claim that when $a=2$ we have $C / R_{u}(C) \geq O_{4}$. So suppose $a=2$ and $V \downarrow v=W(2 m)^{2}$. Then $u=v^{2}$ is in the class of $J_{m}{ }^{4}$ in $G=S L_{n}$. We now work with root groups in the root system of $G$ of type $A_{4 m-1}$, with fundamental roots $\alpha_{1}, \ldots, \alpha_{4 m-1}$. Take $\tau$ to be a standard graph automorphism of $G$. Write $u_{i j k \ldots}=x_{\alpha_{i}+\alpha_{j}+\alpha_{k}+\ldots}(1)$, and define

$$
\begin{aligned}
v^{\prime}= & \tau\left(u_{1234} u_{5678} \ldots u_{4 m-7,4 m-6,4 m-5,4 m-4}\right) \times \\
& \left(u_{2345} u_{6789} \ldots u_{4 m-6,4 m-5,4 m-4,4 m-3}\right) .
\end{aligned}
$$

Then

$$
\begin{aligned}
\left(v^{\prime}\right)^{2}= & \left(u_{1234} u_{5678} \ldots u_{4 m-7,4 m-6,4 m-5,4 m-4}\right) \times \\
& \left(u_{2345} u_{6789} \ldots u_{4 m-6,4 m-5,4 m-4,4 m-3}\right) \times \\
& \left(u_{4 m-4,4 m-3,4 m-2,4 m-1} \ldots u_{4567}\right) \times \\
& \left(u_{4 m-5,4 m-4,4 m-3,4 m-2} \ldots u_{3456}\right),
\end{aligned}
$$

which is in the class $\left(A_{m-1}\right)^{4}$ in $S L_{n}$, hence is conjugate to $u$. Since $W(2 m)^{2}$ is the only $S p(V)$-class squaring to $u$, we may therefore take $v=v^{\prime}$. One checks that $v$ is centralized by the group

$$
\left\langle x_{1}(t) x_{3}(t) \cdots x_{4 m-1}(t), s_{1} s_{3} s_{5} \cdots s_{m-1}: t \in K\right\rangle=A \cong A_{1},
$$

and also by the element

$$
j=s_{2} s_{6} s_{10} \ldots s_{4 m-2} u_{2345} u_{6789} \ldots u_{4 m-6,4 m-5,4 m-4,4 m-3} .
$$

Moreover, $A$ commutes with $A^{j}$, so $C_{G}(v)$ contains $\left\langle A, A^{j}\right\rangle \cong S O_{4}$. Since we already know that $C / R_{u}(C)$ contains $G L_{2} .2$, it follows that $C / R_{u}(C)$ contains $\mathrm{O}_{4}$, as claimed.

Now we return to the case where $a$ is arbitrary, and argue that $C / R_{u}(C)$ contains $O_{2 a}$. To see this, we can suppose that $a \geq 2$ (since if $a=1$ then $C$ contains $G L_{1} .2=O_{2}$ ). Write

$$
V \downarrow v=W(2 m)^{2}+W(2 m)^{a-2},
$$

and let $E_{1}, F_{1}$ be the corresponding pair of singular $4 m$-spaces for the first factor, and $E_{2}, F_{2}$ those for the second. So we can take $G=S L_{2 a m}$ to fix the pair $Y=E_{1}+E_{2}, Z=F_{1}+F_{2}$ of singular spaces in $V$. By the above claim, $C / R_{u}(C)$ contains $O_{4}$ acting on $E_{1}$ and $F_{1}$, and centralizing $E_{2}+F_{2}$. It also contains $G L_{a} .2$ and is contained in $S p_{2 a}$. As the only proper overgroups of $G L_{a} .2$ in $S p_{2 a}$ are $O_{2 a}$ and $S p_{2 a}$, it follows that $C / R_{u}(C)$ contains $O_{2 a}$.

Finally, we argue that $C / R_{u}(C)=O_{2 a}$. Suppose this is not the case. Then $C / R_{u}(C)=S p_{2 a}$. Let $T$ be a torus of rank $a-1$ in $C$ centralizing a subgroup 
$S p_{2}$ of $C / R_{u}(C)$, and let $X$ be a minimal preimage of this $S p_{2}$ in $C_{C}(T)$ (i.e. $\left.X=C_{C}(T)^{(\infty)}\right)$. As above, let $Y, Z$ be a pair of singular spaces in $V$ fixed by $G=S L_{2 a m}$ and interchanged by $v$. Then $Y=C_{Y}(T)+[Y, T]$, and $X$ acts on both these subspaces. In $[Y, T]$ there are $2 a-2$ weight spaces of $T$, each of dimension $m$, and $u=v^{2}$ acts on each as a single Jordan block. As $X$ acts on each weight space, centralizing the action of $u$, it follows that $X$ induces a unipotent group on each weight space, and hence that $X$ acts trivially on $[Y, T]$. Now consider $C_{Y}(T)$. The fixed point space of $T$ on $V$ is $C_{Y}(T)+C_{Y}(T)^{v}$, a non-degenerate $4 m$-space on which $v$ acts as $W(2 m)$. Moreover $C_{G}(v)$ induces $S p_{2}$ modulo its unipotent radical on this space. Hence we have reduced to the case where $a=1$.

So assume that $a=1$. Next, we further reduce to the case where $m=2$. Regard $V \downarrow v$ as $V(2 m) \otimes V_{2}$, with $v$ trivial on $V_{2}$. Here $v \in S p(V(2 m))$ acts on the first factor and is centralized by $S p\left(V_{2}\right)$ acting on the second factor. First note that, given our supposition, $C$ induces $S p_{2}$ on each $C$-composition factor of $V$. This is because $C$ covers $S p\left(V_{2}\right)$ modulo $R_{u}\left(C_{S p(V)}(v)\right)$ and $S p\left(V_{2}\right)$ acts homogeneously on $V$.

Let $R, S$ be singular 1-spaces in $V_{2}$ as before. There is an involution $t \in$ $S p\left(V_{2}\right)$ interchanging $R$ and $S$. Then $\tilde{v}=v t$ interchanges the two singular spaces $W=V(2 m) \otimes R$ and $W^{\prime}=V(2 m) \otimes S$. Also $t$ centralizes $S p\left(V_{2 m}\right)$ and therefore $t$ induces a standard graph automorphism of $G=G L(W)$. Also $(v t)^{2}=v^{2} t^{2}=u$, which induces $J_{m}{ }^{2}$ on $W$. Hence we may replace $v$ by $\tilde{v}=v t$, and take $G=G L(W)$ with $\tau=t$. Then our supposition implies that $\tilde{C}=C_{G}(\tilde{v})$ has reductive part $S p_{2}$ which acts on each $\tilde{C}$-composition factor as a natural module.

Let $F=C_{V(2 m)}(v)$, a 1 -space. Then $C_{V}(v)=F \otimes V_{2}$. As $t$ commutes with $v$, we see that $\tilde{t}$ acts on $C_{V}(v)^{\perp} / C_{V}(v)=\left(F^{\perp} \otimes V_{2}\right) /\left(F \otimes V_{2}\right)$. As $\tilde{v}^{2}=u$, the element which $\tilde{v}$ induces on $V(2 m-2) \otimes V_{2}$ is in the class $W(2 m-2)$, and still has $S p_{2}$ in the reductive part of its centralizer in $G L(V(2 m-2) \otimes R)$. Repeating this argument, we end up with an element in the class $W(4)$ in $S p_{8}$, lying in $G L_{4} .2$, and such that its centralizer in $G L_{4}$ has reductive part $S p_{2}$. Thus we have now reduced to the case where $m=2$.

Now observe that $S L_{4} .2 \cong O_{6}$, and $v$ is in the class $V(4)+W(1)$ of $O_{6}$. Indeed this follows from $[11,(4.4)$ and Theorem 4.2], since $v$ is an outer unipotent element of order 4 centralizing a $T_{1}<S L_{4}$. But then [11, Theorem 4.2] also shows that $C_{\mathrm{SO}_{6}}(v) / R_{u}\left(C_{S_{6}}(v)\right) \cong \mathrm{O}_{2}$ rather than $\mathrm{Sp}_{2}$ for this class. This final contradiction completes the proof.

Lemma 4.5 Let $v \in S L_{n}\langle\tau\rangle<S p(V)$ be such that $V \downarrow v=W(2 m)^{a}+V(2 m \pm$ 2) with $m$ even, and let $C=C_{S L_{n}}(v)$. Then $C^{\circ} / R_{u}(C) \cong S p_{2 a}$.

Proof We know by Lemma 4.4 that $C / R_{u}(C)$ contains $O_{2 a}$, and it is contained in $S p_{2 a}$ since this is the reductive part of the centralizer of $v$ in $S p(V)$ (see [11, Theorem 4.2]). Hence it is enough to show that $C / R_{u}(C)$ contains a long root group of $S p_{2 a}$, and for this it is sufficient to consider the case where $a=1$. We 
do this by induction on the dimension of $V$, the base cases $W(4)+V(2)$ and $W(4)+V(6)$ being handled by Lemma 4.3 .

Assume first that $V \downarrow v=W(2 m)+V(2 m+2)$ with $m>2$. We take $G\langle\tau\rangle=S L(R)\langle v\rangle$, where $R, R^{v}$ are maximal singular spaces in $V$ interchanged by $v$, and $V=R+R^{v}$. Then $\operatorname{dim} R=3 m+1$, and $u=v^{2}$ acts on $R$ as $J_{m+1}+J_{m}{ }^{2}$. We work with root groups in the root system of $G$ of type $A_{3 m}$, with fundamental roots $\alpha_{1}, \ldots, \alpha_{3 m}$. Taking $\tau$ to be a standard graph automorphism, and writing $u_{i j k \ldots}=x_{\alpha_{i}+\alpha_{j}+\alpha_{k}+\ldots(1) \text {, define }}$

$$
v^{\prime}=\tau\left(u_{123} u_{456} \ldots u_{\frac{3}{2} m-2, \frac{3}{2} m-1, \frac{3}{2} m}\right)\left(u_{234} u_{567} \ldots u_{3 m-4,3 m-3,3 m-2}\right) .
$$

One checks that

$$
\begin{aligned}
u^{\prime}=\left(v^{\prime}\right)^{2}= & \left(u_{234} u_{567} \ldots u_{3 m-4,3 m-3,3 m-2}\right) \times \\
& \left(u_{345} u_{678} \ldots u_{3 m-3,3 m-2,3 m-1}\right)^{-1} \times \\
& \left.\left(u_{123} u_{456} \ldots u_{\frac{3}{2} m-2, \frac{3}{2} m-1, \frac{3}{2} m}\right)^{\tau}\right) \times \\
& \left(u_{123} u_{456} \ldots u_{\frac{3}{2} m-2, \frac{3}{2} m-1, \frac{3}{2} m}\right) .
\end{aligned}
$$

Then $u^{\prime}=\left(v^{\prime}\right)^{2}$ is in the class $A_{m}\left(A_{m-1}\right)^{2}$ of $G$, hence is conjugate to $u$. So we may take $v=v^{\prime}$ and $u=u^{\prime}$. Let $T$ be the 1 -dimensional torus described in the discussion preceding Lemma 3.4 which acts with weight 2 on each root element $e_{i j k}$ corresponding to a root group element $u_{i j k}$ appearing in the expression for $u$. The corresponding labelled Dynkin diagram $\Delta$ has label 0 on the nodes $\alpha_{2}, \alpha_{5}, \alpha_{8}, \ldots, \alpha_{3 m-1}$ and 1 on the other nodes. Let $P=Q L$ be the parabolic subgroup of $G$ determined by $\Delta$, such that $L=C_{G}(T)$ and $Q$ is the product of all root groups for a maximal torus in $L$ having positive $T$-weight. By Lemma 3.4, $C_{G}(u)<P$.

For $c \in K$, define

$$
t(c)=x_{-2}(c) x_{-5}(c) x_{-8}(c) \ldots x_{-(3 m-1)}(c),
$$

a root element in a diagonal $A_{1}$ subgroup of $L$. We shall see that this element can be adjusted to centralize $v$. Setting

$$
r(c)=x_{34}(c) x_{67}(c) \ldots x_{\frac{3 m}{2}-3, \frac{3 m}{2}-2}(c),
$$

one checks that

$$
v^{t(c) r(c)}=v x_{\frac{3 m}{2}, \frac{3 m}{2}+1}(c) .
$$

Then $v^{t(c) r(c) s(d)}=v$, where $s(d)=u_{\frac{3 m}{2}}(d) u_{\frac{3 m}{2}+1}(d)$ and $d^{2}=c$. It follows that $C Q / Q$ contains a root group of a diagonal $A_{1}$ of the Levi factor. Now $C Q / Q<C_{G}(u) Q / Q$ which is $A_{1} T_{1}$ by [11, Theorems 1 and 3.1]. Therefore $C Q / Q$ contains $\left\langle O_{2}, t(c): c \in K\right\rangle \cong S p_{2}$, as required.

Now consider the case where $V \downarrow v=W(2 m)+V(2 m-2)$ with $m>2$. The argument is very similar to the above. Here $u=v^{2}$ acts on the singular space $R$ as $J_{m-1}+J_{m}{ }^{2}$. Working in the root system of $G$ of type $A_{3 m-2}$, we may take

$$
v=\tau\left(u_{123} u_{456} \ldots u_{3 m-5,3 m-4,3 m-3}\right)\left(u_{345} u_{678} \ldots u_{\frac{3}{2} m-3, \frac{3}{2} m-2, \frac{3}{2} m-1}\right) .
$$


The 1-dimensional torus $T$ has labelled Dynkin diagram $\Delta$ with label 0 on the nodes $\alpha_{1}, \alpha_{4}, \alpha_{7}, \ldots, \alpha_{3 m-2}$ and 1 on the other nodes. Write $P=Q L$ for the parabolic subgroup determined by $\Delta$ as above. Define

$$
t(c)=x_{-1}(c) x_{-4}(c) x_{-7}(c) \ldots x_{-(3 m-2)}(c)
$$

and

$$
r(c)=x_{23}(c) x_{56}(c) \ldots x_{\frac{3 m}{2}-4, \frac{3 m}{2}-3}(c) .
$$

Then

$$
v^{t(c) r(c)}=v x_{\frac{3 m}{2}-1, \frac{3 m}{2}}(c) .
$$

Finally, $v^{t(c) r(c) s(d)}=v$, where $s(d)=x_{\frac{3 m}{2}-1}(d) x_{\frac{3 m}{2}}(d)$ and $d^{2}=c$. As before this yields the assertion, completing the proof.

Lemma 4.6 Let $v \in S L_{n}\langle\tau\rangle<S p(V)$ be such that $V \downarrow v=W(2 m)^{a}+$ $\sum V\left(2 k_{i}\right)^{c_{i}}$ with $m$ even, $k_{i} \neq m \pm 1$ for all $i, k_{1}>k_{2}>\cdots$, and $c_{i} \leq 2$ for each $i$. If $C=C_{S L_{n}}(v)$, then $C^{\circ} / R_{u}(C) \cong S O_{2 a}$.

Proof Lemma 4.4 implies that $C^{\circ} / R_{u}(C)$ contains $S O_{2 a}$, and it is contained in $S p_{2 a}$ since this is the reductive part of the centralizer of $v$ in $S p(V)$. Hence $C^{\circ} / R_{u}(C)$ is $S p_{2 a}$ or $S O_{2 a}$. We proceed by induction assuming $V$ is a counterexample of minimal dimension. That is, suppose that $C^{\circ} / R_{u}(C) \cong S p_{2 a}$.

If $a>1$, let $T_{a-1} \times T_{1}$ be a maximal torus of $S O_{2 a}$ (acting on $W(2 m)^{2 a}$ ) such that $T_{a-1} \leq S O_{2 a-2}$ and $T_{1}=S O_{2}$. Then $C_{C}\left(T_{a-1}\right)$ acts on $C_{V}\left(T_{a-1}\right)=$ $W(2 m)+\sum V\left(2 k_{i}\right)$. Also $C_{C}\left(T_{a-1}\right)$ contains $T_{1}$ and has a quotient of type $S p_{2}$. So by restricting to $C_{V}\left(T_{a-1}\right)$ it is enough to obtain a contradiction for the case $V \downarrow v=W(2 m)+\sum V\left(2 k_{i}\right)^{c_{i}}$ with $C^{\circ} / R_{u}(C) \cong S p_{2}$.

In the course of the proof to follow we will produce certain sections $A / B$ of $V$ on which $v$ and $C$ act, and then contradict minimality. We note the reductive part of the action of $C$ will still contain $S p_{2}$, provided the summand $W(2 m)$ is not contained in $B$. This follows since a maximal torus of $C$ acts without fixed points on $W(2 m)$ (and acts trivially on all other summands).

Assume that $m>2$. If $J$ is a Jordan block for $v$ of size greater than 2 in the above decomposition, then $[J, v]^{(2)}=[J, u]$ has codimension 2 and $C_{J}(u)$ has dimension 2. The quotient is a section of $J$ of dimension 4 less than that of $J$. Now do this for all of $V$. Let $X=[V, v]^{(2)}=[V, u]$ and $Y=C_{[V, u]}(u)$. Then $C$ acts on $X / Y$, a non-degenerate space such that $(X / Y) \downarrow$ $v=W(2 m-4)+\sum V\left(2 k_{i}-4\right)^{c_{i}}$, where the sum is over those $i$ for which $k_{i}>1$. Moreover, $C^{\circ}$ contains a factor $S p_{2}$ in its action on $X$, which contradicts the minimality of $\operatorname{dim} V$. Hence we reduce to the case $m=2$, where by hypothesis, $k_{i} \neq 1,3$. Therefore, we now have $V \downarrow v=W(4)+\sum V\left(2 k_{i}\right)^{c_{i}}$, with $k_{i} \geq 5$ for each $i$.

If $k_{1}>5$, we can again use the inductive hypothesis to get a contradiction. Indeed, let $W=[V, v]^{\left(2 k_{1}-2\right)}$. Then $W=C_{V\left(2 k_{1}\right)^{c_{1}}}(u)$ is a sum of $c_{1}$ Jordan blocks $J_{2}$ for $v$ in $V\left(2 k_{1}\right)^{c_{1}}$. Now consider the action of $v$ on $W^{\perp} / W$. There 
is a complication if $k_{1}-2=k_{2}$ and $c_{1}+c_{2}>2$. If this does not occur, then we contradict the minimality of $\operatorname{dim} V$. So consider the exceptional case where $\left(W^{\perp} / W\right) \downarrow v=W(4)+W\left(2 k_{2}\right)+V\left(2 k_{2}\right)^{c_{1}+c_{2}-2}+\sum_{i>2} V\left(2 k_{i}\right)^{c_{i}}$. The proof of Lemma 4.4 shows that the centralizer of the induced action of $v$ contains subgroups $E=S p_{2}$ and $\mathrm{O}_{2}$ acting faithfully on $W\left(2 k_{2}\right)$ and $W(4)$, respectively, and acting trivially on the remaining factors. As these groups commute, the reductive part of the centralizer of the induced action of $v$ is $S p_{2} \times S p_{2}$ with $C^{\circ}$ covering one of the factors and $E$ covering the other. So if $D$ is a maximal torus of $E$, then the centralizer of $D$ acts on $\left[W^{\perp} / W, D\right]$ and has reductive part $S p_{2}$. Hence we obtain a smaller counterexample on a space where $v$ acts as $W(4)+V\left(2 k_{2}\right)^{c_{1}+c_{2}-2}+\sum_{i>2} V\left(2 k_{i}\right)^{c_{i}}$.

The final case to consider is that where $V \downarrow v=W(4)+V(10)^{a}$. Here we set $S=[V, v]^{(5)}$, a maximal singular subspace of the summand $V(10)^{a}$. Then $\left(S^{\perp} / S\right) \downarrow v=W(4)$ and $C$ acts such that the reductive part of the induced action is $S p_{2}$, contradicting Lemma 4.4.

\subsection{Connected centralizers in Theorem 1.2}

We now start work on the proof of Theorem 1.2 in general. Thus we continue to assume $G=S L_{n}(K)$ with $G\langle\tau\rangle<S p(V)$; let $v \in G \tau$, and as in (1) write

$$
V \downarrow v=\sum_{m_{i} \text { odd }} W\left(2 m_{i}\right)^{a_{i}}+\sum_{n_{i} \text { even }} W\left(2 n_{i}\right)^{b_{i}}+\sum_{k_{i} \text { odd }} V\left(2 k_{i}\right),
$$

where the $m_{i}$ and $n_{i}$ are distinct, and the $k_{i}$ are in non-increasing order and occur with multiplicity at most 2 . Let $C=C_{G}(v)$ and $\bar{C}=C / R_{u}(C)$.

We now identify the connected reductive group $\bar{C}^{\circ}$. This contains $\prod S p_{2 a_{i}} \times$ $\prod S O_{2 b_{i}}$ by Lemma 4.4, and is contained in $\prod S p_{2 a_{i}} \times \prod S p_{2 b_{i}}$ (since this is the connected reductive part of the centralizer of $v$ in $S p(V))$. So

$$
\bar{C}^{\circ}=\prod S p_{2 a_{i}} \times \prod I_{2 b_{i}}
$$

where each $I_{2 b_{i}}$ is $S p_{2 b_{i}}$ or $S O_{2 b_{i}}$. Let $T^{(i)}$ be a maximal torus of the product of all factors of $\bar{C}^{\circ}$ apart from $I_{2 b_{i}}$, and let $C_{i}=C_{C}\left(T^{(i)}\right)$. Then $C_{i}^{\circ} / R_{u}\left(C_{i}\right) \cong I_{2 b_{i}}$, and $C_{i}$ acts on $C_{V}\left(T^{(i)}\right)$, which is just the space $W\left(2 n_{i}\right)^{b_{i}}+\sum V\left(2 k_{i}\right)$. Hence by Lemmas 4.5 and 4.6 , we have $I_{2 b_{i}}=S p_{2 b_{i}}$ if there exists $k_{j}=n_{i} \pm 1$, and $I_{2 b_{i}}=S O_{2 b_{i}}$ otherwise. Hence $\bar{C}^{\circ}$ is as in the statement of Theorem 1.2(iii).

\subsection{Component groups of centralizers in Theorem 1.2}

Continuing with the notation of the previous subsection, to complete the proof of Theorem 1.2 it remains to find the component group $C / C^{\circ}$ of $C=C_{G}(v)$. This is given in [16, p.24]. However, there is a small error there, so we shall offer an independent proof. Let $v$ and $u$ be as in equations (1) and (2) of subsection 1.3 and let $\lambda$ and $\epsilon$ be as described in the discussion prior to Lemma 4.2. 
Let us first explain the assertions and the error in [16, p.24]. Relabel the partition of $n$ given by (2) as $\left(\lambda_{1}, \lambda_{2}, \lambda_{3}, \ldots\right)$ with $\lambda_{1} \geq \lambda_{2} \geq \cdots$, so $E \downarrow u=$ $\sum J_{\lambda_{i}}$. Then the component group $C / C^{\circ}$ is the abelian group generated by a set of involutions $\left\{a_{i}: \epsilon\left(\lambda_{i}\right) \neq 0\right\}$, subject to the following relations: $a_{i} a_{j}=1$ if $\lambda_{i}=\lambda_{j}$, or if $\lambda_{i}=\lambda_{j}+1$, or if $\lambda_{i}$ is odd and $\lambda_{i}=\lambda_{j}+2$; and also $a_{i}=1$ if $\lambda_{i}=1$. The error in [16] is that the last relation is omitted. One checks that this description of the component group agrees with the one given by Theorem 1.2.

We now present our proof that the component group $C / C^{\circ}$ is as asserted in Theorem 1.2. As before, regard $S L_{n} .2=S L_{n}\langle\tau\rangle<S p_{2 n}$, where $\tau$ is the standard graph automorphism of $G=S L_{n}$ with respect to a fixed root system. Let $V$ be the natural module for $S p_{2 n}$ and write $V=E \oplus E^{\tau}$, where $G=S L_{n}$ acts on each of the totally singular summands. Recall that we have a nondegenerate bilinear form $(,)_{v}$ on $E$, where $\left(e_{1}, e_{2}\right)_{v}=\left(e_{1}, e_{2}{ }^{v}\right)$.

Let $S<E$. Then $S$ is a singular space under the form $(,)_{v}$ if and only if $S+S^{v}$ is singular in $V$. Let $S^{\perp_{v}}$ denote the annihilator in $E$ of $S$ under $(,)_{v}$.

Let $v \in G \tau$ with $u=v^{2}$. Write $V \downarrow v$ and $E \downarrow u$ as in (1) and (2), respectively. We shall see in Lemma 4.9 to follow that the proof ultimately reduces to the case where $V \downarrow v$ is distinguished. That is,

$$
V \downarrow v=\sum_{k_{i} \text { odd }} V\left(2 k_{i}\right)
$$

and thus

$$
E \downarrow u=\sum_{k_{i} \text { odd }} J_{k_{i}},
$$

where for each $i$ the Jordan block $J_{k_{i}}$ can be taken such that $J_{k_{i}}+J_{k_{i}}{ }^{v}=$ $V\left(2 k_{i}\right)$. This follows from the proof of Lemma 4.1 and the classification of outer unipotent elements.

In this situation we choose a Levi subgroup $L$ with respect to the given root system such that $L^{\prime}=\prod A_{k_{i}-1}$. Let $w_{0}$ be the long word in the Weyl group of $G$, and for each $i$ let $w_{i, 0}$ be the long word in the Weyl group of $A_{k_{i}-1}$. Define

$$
\gamma=\tau w_{0} \prod w_{i, 0}
$$

Then $\gamma$ induces a standard graph automorphism on each $A_{k_{i}-1}$.

For $k_{i}>1$, let $A_{k_{i}-1}$ have fundamental system $\alpha_{i, 1}, \ldots, \alpha_{i, k_{i}-1}$, and let $x_{i}=u_{i, 1} \ldots u_{i, \frac{k_{i}-1}{2}}$, where $u_{i, j}$ is the root element $x_{i, j}(1)$. If $v^{\prime}=\gamma x_{1} x_{2} \ldots$, then $\left(v^{\prime}\right)^{2}=u_{1} u_{2} \ldots$, where each $u_{i}$ is a regular unipotent element in $A_{k_{i}-1}$. It follows that $v^{\prime}$ is conjugate to $v$ and so we can assume $v=v^{\prime}$ and $u=u_{1} u_{2} \ldots$ in the following. Notice that $\prod C_{A_{k_{i}-1}}(v) \leq C_{G}(v)$. Further, for each pair $k_{i}$, $k_{j}$, if we view $A_{k_{i}-1} A_{k_{j}-1}<A_{k_{i}+k_{j}-1}$, then $C_{A_{k_{i}+k_{j}-1}}(v)^{\circ}<C_{G}(v)^{\circ}$.

Lemma 4.7 Assume $V \downarrow v=\sum_{k_{i} \text { odd }} V\left(2 k_{i}\right)$ and let notation be as above. If $k_{i}>1$, then $C_{A_{k_{i}-1}}(v)=U_{\frac{k_{i}-1}{2}} .2$, with the component group generated by $u_{i}$. 
Proof Simplify notation by considering $A_{r}$ for $r$ even and $v=\tau u_{1} \ldots u_{\frac{r}{2}}$ squaring to a regular element $u \in A_{r}$. Then setting $u=v^{2}$, we see that $E \downarrow u=J$ is a Jordan block of size $r+1$ and the dimension formula (3) gives $C_{A_{r}}(v)^{\circ}=U_{\frac{r}{2}}$. (This can be proved directly. Namely, $C_{A_{r}}(u)$ is equal to $U_{r}$, an abelian group. The map $\phi: U_{r} \rightarrow U_{r}$ sending $d \mapsto d^{v}=d^{\tau}$ has its image in its kernel which is $C_{U_{r}}(v)$. This implies that $\operatorname{dim} C_{A_{r}}(v) \geq \frac{r}{2}$. The rest of the argument below will give the equality.)

Proceed by induction. The base case $r=2$ is an easy calculation, so assume $r>2$. Let $X_{\beta}$ be the root subgroup corresponding to the highest root $\beta=$ $11 \ldots 11$. Then $X_{\beta} \leq C_{G}(v)$ and $v$ acts on $P=N_{G}\left(X_{\beta}\right)=Q L$, where $L^{\prime}=$ $A_{r-2}, Q^{\prime}=X_{\beta}$, and $C_{Q / Q^{\prime}}(v)=\left\langle x_{11 \ldots 110}(c) x_{011 \ldots 11}(c) Q^{\prime}: c \in K\right\rangle$. However, $\left(x_{11 \ldots 110}(c) x_{011 \ldots 11}(c)\right)^{v}=x_{11 \ldots 110}(c) x_{011 \ldots 11}(c) x_{111 \ldots 11}(c)$, so that $C_{Q}(v)=Q^{\prime}$.

Now pass to $P / Q$ where $u Q=(v Q)^{2}$ is a regular unipotent element in $L Q / Q \cong A_{r-2}$. Inductively, $C_{L Q / Q}(v Q)=U_{\frac{r-2}{2}} .2$, with $u Q$ generating the component group. Then $C_{G}(v) Q \leq C_{P / Q}(v Q)^{2}$, so the assertion follows by induction and the previous paragraph.

Lemma 4.8 Assume $V \downarrow v=\sum_{k_{i} \text { odd }} V\left(2 k_{i}\right)$ is distinguished. Then $C_{G}(v)=$ $C_{G}(v)^{\circ}\left\langle u_{i}: k_{i}>1\right\rangle$ and $C_{G}(v) / C_{G}(v)^{\circ}$ is abelian of exponent at most 2 .

Proof The assertion follows from Lemma 4.7 if $V=V\left(2 k_{1}\right)$ with $k_{1}>1$. And if $V=V(2)$, then $G=1$ and again the assertion holds. So now assume there is more than one summand and set $W=J_{k_{1}}$, where $J_{k_{1}}+J_{k_{1}}{ }^{v}=V\left(2 k_{1}\right)$. That is, $J_{k_{1}}$ is non-degenerate under $(,)_{v}$. Let $D=C_{G L(E)}(u)$ and $M=W^{D}$. Then $M=S \cup N$, where $N$ consists of the non-degenerate Jordan blocks of $u$ on $E$ and $S$ consists of the blocks with nonzero radical. So $N$ is open and dense in $M$. As $D$ is connected, $M$ is irreducible.

We claim that $C=C_{G}(v)$ is transitive on $M$. Suppose $J, J^{\prime} \in M$ and write $E=J \perp X=J^{\prime} \perp X^{\prime}$. Then $R=J+J^{v}$ and $R^{\prime}=J^{\prime}+J^{\prime v}$ are non-degenerate in $V$ of type $V\left(2 k_{1}\right)$.

We next consider the action of $v$ on $R$. To simplify notation for just this paragraph we will regard $V=R=V\left(2 k_{1}\right)$. In the proof of Lemma 4.1 we gave a basis $\mathcal{B}=\left\{v_{2 k_{1}}, \ldots, v_{1}\right\}$ with corresponding inner products, and the precise action of $v$ and corresponding action of $u$ on a pair of maximal singular spaces interchanged by $v$. One singular space is the Jordan block of $u$ generated by $v_{2 k_{1}}$; the other is its image under $v$. By [11, Theorem 4.2] the reductive part of $Z=C_{S p(R)}(u)^{\circ}$ is a 1-dimensional torus. The 1-dimensional tori of $Z$ are all conjugate and each determines a unique pair of totally singular spaces. One such torus determines the pair $J, J^{v}$. Therefore, conjugating by an element $g \in Z$, we can carry the above basis to the action of $v^{g}$ on $R$ with $J, J^{v}$ being the singular spaces interchanged by $v^{g}$. Then there exists $k \in S L(J)$ such that $v=v^{g k}$. Therefore there is a basis $\mathcal{B}^{g k}$ of $R$ such that the action of $u$ on $J$, the action of $v$ on $R$ and the form on $R$ are precisely as in the proof of Lemma 4.1.

Returning to the general case, a corresponding basis exists for $R^{\prime}=J^{\prime}+J^{\prime v}$. Write $V=\left(J+J^{v}\right) \perp\left(X+X^{v}\right)$ and similarly $V=\left(J^{\prime}+J^{\prime v}\right) \perp\left(X^{\prime}+X^{\prime v}\right)$. 
Decompose $X+X^{v}$ and $X^{\prime}+X^{\prime v}$ and repeat the above argument. An application of Witt's theorem then yields an element $y \in C_{S p(V)}(v)$ sending one basis to the next. In particular $y$ sends $J$ to $J^{\prime}$ and also $J+X$ to $J^{\prime}+X^{\prime}$, and hence $y \in G Z$, where $Z$ is a torus inverted by $v$. As $y$ centralizes $v$, we have $y \in C$ and the claim follows.

Now taking $W \in M$, the orbit $W^{C^{\circ}}$ is open in its closure in $M$. The closure must be $M$, as otherwise $M$ would be a union of finitely many such orbit closures. Hence $W^{C^{\circ}}$ is open dense in $M$. There are only finitely many such orbits (since $C$ is transitive on $M$ and $C / C^{\circ}$ is finite), so each must be closed as well. But this also contradicts irreducibility, unless $C^{\circ}$ is transitive on $M$. Hence, $C^{\circ}$ is transitive on $M=W^{C}$ and we can write $C=C^{\circ} \operatorname{Stab}_{C}(W)$. Writing $E=W \perp_{v} Y$ we have $\operatorname{Stab}_{C}(W)=C_{W} \times C_{Y}$, where $C_{W}$ is the centralizer of $v$ in $S L(W)$ viewed as a subgroup of $S p\left(W+W^{v}\right)=S p\left(2 k_{1}\right)$, and $C_{Y}$ is the centralizer of $v$ in $S L(Y)<S p\left(Y+Y^{v}\right)$. Lemma 4.7 and induction now yield the result.

Lemma 4.9 It suffices to establish Theorem 1.2 when $v$ is distinguished in $S p(V)$.

Proof By Lemmas 4.4, 4.5, and 4.6

$$
C / R_{u}(C) \geq \prod_{m_{i} \text { odd }} S p_{2 a_{i}} \times \prod_{n_{i} \text { even }} I_{2 b_{i}}
$$

where $I_{2 b_{i}}=O_{2 b_{i}}$ or $S p_{2 b_{i}}$.

Write $V=V_{c}+V_{d}$, where $V_{c} \downarrow v=\sum W\left(2 m_{i}\right)^{a_{i}}+\sum W\left(2 n_{i}\right)^{b_{i}}$ and $V_{d}=$ $\sum V\left(2 k_{i}\right)$. Let $T_{0}$ be a maximal torus of $C=C_{G}(v)$, so that $T_{0}$ projects to a maximal torus of $C^{\circ} / R_{u}(C)$ and a Frattini argument gives $C=C^{\circ} N_{C}\left(T_{0}\right)$. By Lemma 4.4 we can choose $T_{0}$ to act trivially on $V_{d}$. Then $V_{d}=\left[V, T_{0}\right]$ so that $N_{C}\left(T_{0}\right)=N_{c} \times N_{d}$, the induced actions on $V_{c}$ and $V_{d}$ respectively.

Moreover, $N_{C}\left(T_{0}\right)$ permutes the homogeneous components of the action of $T_{0}$ on $V$. Each such component is invariant under the action of $v$, so $N_{C}\left(T_{0}\right)$ permutes components on which $v$ has Jordan blocks of a given size. It follows that $N_{C}\left(T_{0}\right)$ preserves the decomposition $V_{c}=\sum V_{i}$, where each summand is one of the $W\left(2 m_{i}\right)^{a_{i}}$ or $W\left(2 n_{i}\right)^{b_{i}}$ summands and $T_{0}$ is a corresponding product of subtori, each acting on a single summand (of dimension $4 m_{i} a_{i}$ or $4 n_{i} b_{i}$ ).

For each $i$, the group $N_{C}\left(T_{0}\right)$ acts on the maximal singular spaces $E_{i}=V_{i} \cap E$ and $E_{i}{ }^{v}$, and we let $N_{i}$ denote the restriction of $N_{d}$ to the summand $V_{i}$. Then $N_{C}\left(T_{0}\right)=N_{c} \times N_{d}$ and $N_{c}=\prod N_{i}$, where $N_{i}=N_{C_{S L\left(E_{i}\right)}(v)}\left(T_{0}\right)$. Also, $N_{d}$ centralizes $T_{0}$, since $T_{0}$ acts trivially on $V_{d}$.

It follows from Lemma 4.4 that for each $i$ we have $N_{i} / R_{u}\left(N_{i}\right)=T_{i} Z_{2}{ }^{a_{i}}{ }^{S y m_{a_{i}}}$ or $T_{i} Z_{2}{ }^{b_{i}} \operatorname{Sym}_{b_{i}}$ as appropriate, and that $N_{i} /\left(N_{i} \cap C_{S L\left(E_{i}\right)}(v)\right)^{\circ}$ has order at most 2. (It may happen that the order is 2 but $N_{i}<C^{\circ}$ due to Lemma 4.5.) Also Lemma 4.8 shows that $N_{d} / N_{d}{ }^{\circ}$ is elementary abelian. Therefore $C / C^{\circ}$ is elementary abelian. Since $C^{\circ} / R_{u}(C)$ is a product of symplectic and special orthogonal groups, each normalized by $N_{C}\left(T_{0}\right)$, we have $C / R_{u}(C)=\prod_{m_{i} \text { odd }} S p_{2 a_{i}} \times$ 
$\prod_{n_{i} \text { even }} I_{2 b_{i}} \times Z$, where $Z$ is elementary abelian. A Frattini argument shows that $Z$ is covered by the component group of $C_{C}\left(T_{0}\right)$. Now $C_{C}\left(T_{0}\right)$ acts on $V_{c}$ and $V_{d}$ so that $C_{C}\left(T_{0}\right)=C_{c} \times C_{d}=C_{c} \times N_{d}$. Also $C_{C}\left(T_{0}\right) \cap C^{\circ}=T_{0} \times C_{R_{u}(C)}\left(T_{0}\right)$, which is connected. Moreover $C_{c}$ is connected as it is contained in $R_{u}\left(N_{d}\right) T_{0}$. It follows that $Z$ is isomorphic to the component group of $N_{d}$ which thus lifts faithfully to the component group of $C$. The result follows.

In view of Lemma 4.9 we now assume $V \downarrow v$ to be distinguished. Therefore, $V \downarrow v=\sum_{k_{i} \text { odd }} V\left(2 k_{i}\right)$ and $E \downarrow u=\sum_{k_{i} \text { odd }} J_{k_{i}}$, where $J_{k_{i}}+J_{k_{i}}{ }^{v}=V\left(2 k_{i}\right)$ for each $i$. Then $C_{G}(v) \leq C_{S p(V)}(v)$ is a unipotent group.

We first settle a few small cases which will be needed in inductive arguments to follow.

Lemma 4.10 Assume $(G, u)=\left(S L_{4}, J_{3}+J_{1}\right),\left(S L_{6}, J_{5}+J_{1}\right)$ or $\left(S L_{6}, J_{3}+J_{3}\right)$. Then $C_{G}(v) / C_{G}(v)^{\circ} \cong 1, Z_{2}$ or $Z_{2}$, respectively. In the latter two cases the component group is generated by the image of $u_{1}$, and in the last case $u \in$ $C_{G}(v)^{\circ}$.

Proof In the first case we can consider $S L_{4}\langle\tau\rangle$ as $O_{6}$. Then $v$ is an outer unipotent element of order 8. Using [11, (4.4) and Theorem 4.2], we see that the component group of $C_{O_{6}}(v)$ is $Z_{2}$ (generated by $v$ ), so $C_{G}(v)$ is connected. For the other two cases $G=S L_{6}$ and we will determine the centralizers explicitly.

In the notation given in the paragraphs preceding Lemma 3.4, the labelled Dynkin diagram $\Delta$ corresponding to the Jordan decomposition $J_{5}+J_{1}$ is 22022 ; let $P$ be the parabolic subgroup determined by $\Delta$. Set $v=\tau u_{3} u_{2} u_{1}$, so that $u=$ $v^{2}=u_{4} u_{34} u_{5} u_{2} u_{1}$. A direct check shows that $u$ does have Jordan decomposition $J_{5}+J_{1}$, so this forces $V \downarrow v$ to be distinguished of type $V(10)+V(2)$.

By Lemma 3.4, $C_{G}(v) \leq C_{G}(u) \leq P$. From the form of $v$ it is immediate that $C_{P / Q}(v)<T_{G} X_{\alpha_{3}} Q$ and as $C_{G}(v)$ is unipotent, this forces $C_{G}(v)<U$. Using the computer program described in the appendix we find that $C_{U}(v)$ consists of all elements

$$
\begin{aligned}
& x_{1}(\zeta) x_{5}(\zeta) x_{2}(\zeta) x_{4}(\zeta) x_{12}\left(t_{1}\right) x_{45}\left(t_{1}\right) x_{34}(\zeta) x_{123}\left(t_{2}\right) x_{345}\left(t_{1}+t_{2}\right) \times \\
& x_{234}\left(t_{1}+t_{2}\right) x_{1234}\left(\zeta t_{1}+t_{1}{ }^{2}+t_{1}+t_{2}\right) x_{2345}\left(t_{1}^{2}\right) x_{12345}\left(t_{3}\right),
\end{aligned}
$$

where $\zeta$ is in the prime field and $t_{1}, t_{2}, t_{3}$ range over $K$. Working modulo $Q$ we have $C_{P / Q}(v) \leq T_{G} U_{3}$, where $U_{3}$ is a connected unipotent group of dimension 3 . From this it is easy to see that $C_{G}(v) \leq Q$. From the expression above we see that $C_{G}(v)^{\circ}=U_{3}$ and this is the group obtained from taking $\zeta=0$. Therefore, $C_{G}(v) / C_{G}(v)^{\circ}=Z_{2}$ as asserted.

For the last case the labelled Dynkin diagram $\Delta$ corresponding to the Jordan decomposition $J_{3}+J_{3}$ is 02020 ; let $P=Q L$ be the parabolic subgroup determined by $\Delta$. Let $v=\tau u_{00100} u_{11000} u_{01100}$ so that $u=v^{2}=u_{3} u_{45} u_{34} u_{3} u_{12} u_{23}=$ $u_{45} u_{345} u_{34} u_{12} u_{23}$ lies in $Q$. Note that $x=u^{u_{5}}=\left(u_{34} u_{12}\right)\left(u_{45} u_{23}\right)$ which is clearly of type $J_{3}+J_{3}$. However this does not by itself determine the class of 
$v$, since both types $W(6)$ and $V(6)^{2}$ have square in the class of $u$. This will be settled shortly. Here we find that $C_{U}(v)$ consists of all elements

$$
\begin{aligned}
& x_{1}\left(t_{1}\right) x_{5}\left(t_{1}\right) x_{2}(\zeta) x_{4}(\zeta) x_{3}\left(t_{1}\right) x_{12}\left(t_{2}\right) x_{45}\left(t_{2}\right) x_{23}\left(\zeta t_{1}+\zeta+t_{2}\right) x_{34}\left(\zeta t_{1}+t_{2}\right) \times \\
& x_{123}\left(t_{2}{ }^{2}+t_{2}\right) x_{345}\left(t_{2}^{2}\right) x_{234}\left(t_{3}\right) x_{1234}\left(t_{4}\right) x_{2345}\left(\zeta t_{2}+t_{2}+t_{4}\right) x_{12345}\left(t_{5}\right),
\end{aligned}
$$

where $\zeta$ is in the prime field and $t_{1}, \ldots, t_{5}$ range over $K$. Therefore $C_{Q}(v)^{\circ}=U_{4}$. If $v$ had type $W(6)$, then in view of the dimension information and Lemma 4.4 it would follow that $C_{G}(v)^{\circ}=U_{4} S p_{2}$. Now $C_{G}(v)<C_{G}(u) \leq P$. Further $C_{G}(u) / C_{Q}(u) \cong S L_{2}$. With $x$ as above, $x$ is centralized by the untwisted diagonal $A_{1}=\left\langle x_{1}(t) x_{3}(t) x_{5}(t), x_{-1}(t) x_{-3}(t) x_{-5}(t): t \in K\right\rangle$ in the Levi factor, so that $u$ is centralized by this group conjugated by $u_{5}$. However, $v$ does not centralize this group modulo $Q$. Therefore $V \downarrow v$ is indeed distinguished of type $V(6)^{2}$ and the dimension formula implies that $C_{G}(v)^{\circ}=U_{5}$.

Now $C_{G}(v)=C_{P}(v)$ normalizes $C_{G}(v)^{\circ} Q / Q=\left\langle x_{1}(c) x_{3}(c) x_{5}(c): c \in\right.$ $K\rangle Q / Q$. As $C_{G}(v)$ is unipotent and normalizes $C_{G}(v)^{\circ} Q / Q$ it follows that $C_{G}(v)=C_{U}(v)$. Therefore, $C_{G}(v)$ has component group $Z_{2}$, generated by $u_{1}=u_{23} u_{45}$. Also $u \in C_{G}(v)^{\circ}$.

The next two results will be used in certain inductive arguments.

Lemma 4.11 Assume that $S<C_{E}(u)$ is singular under (, $)_{v}$. Set $\bar{E}=S^{\perp_{v}} / S$ and $\bar{V}=\left(S+S^{v}\right)^{\perp} /\left(S+S^{v}\right)$. Then $S^{\perp_{v}}=E \cap\left(S^{v}\right)^{\perp}$, and the following hold:

(i) there is a natural embedding $\bar{E}<\bar{V}$ as a maximal totally singular subspace (under the induced symplectic form) such that $\bar{V}=\bar{E}+\bar{E}^{v}$;

(ii) $P_{S}=\operatorname{stab}_{G}(S) \cap \operatorname{stab}_{G}\left(S^{\perp_{v}}\right)$ is a v-invariant parabolic subgroup of $G$ which acts on each of $\bar{V}, \bar{E}$ and $\bar{E}^{v}$;

(iii) there is a v-invariant factor of $P_{S} / R_{u}\left(P_{s}\right)$ which induces $S L(\bar{E})$ on each of $\bar{E}$ and $\bar{E}^{v}$.

Proof For $s \in S$ and $e \in E$,

$$
(s, e)_{v}=\left(s, e^{v}\right)=\left(s^{u}, e^{v}\right)=\left(s^{v}, e\right)=\left(e, s^{v}\right),
$$

so that $S^{\perp_{v}}=E \cap\left(S^{v}\right)^{\perp}$. Therefore $S^{\perp_{v}}=E \cap\left(S+S^{v}\right)^{\perp}$ and this yields an embedding $\bar{E} \leq \bar{V}$. Taking images under $v$ we obtain (i).

From the previous paragraph, $\operatorname{stab}_{G}\left(S^{\perp_{v}}\right)=\operatorname{stab}_{G}\left(E \cap\left(S^{v}\right)^{\perp}\right)=\operatorname{stab}_{G}\left(S^{v}\right)$. Therefore, $\operatorname{stab}_{G}(S) \cap \operatorname{stab}_{G}\left(S^{\perp_{v}}\right)=\operatorname{stab}_{G}(S) \cap \operatorname{stab}_{G}\left(S^{v}\right)=P_{S}$ is a $v$-invariant parabolic subgroup of $G$. Also $P_{S}$ acts on $\bar{V}$ and stabilizes $\bar{E}$ and $\bar{E}^{v}$. Moreover, $P_{S}{ }^{\prime}$ has a $v$-invariant Levi factor inducing $S L(\bar{E})$ on $\bar{E}$. Parts (ii) and (iii) now follow.

Lemma 4.12 Assume $V \downarrow v=\sum_{k_{i} \text { odd }} V\left(2 k_{i}\right)$ is distinguished and $E \downarrow u=$ $\sum_{k_{i} \text { odd }} J_{k_{i}}$, as above. Let $Y \leq E$ be a sum of some of the blocks $J_{k_{i}}$ with $k_{i}>1$ and let $S=C_{Y}(u)$. Then $S$ is singular in $E$ with respect to $(,)_{v}$, and with notation as in Lemma 4.11 the following hold: 
(i) $\bar{E} \downarrow u=\sum_{l_{i} \text { odd }} J_{l_{i}}$ (an orthogonal sum under (, ) $)_{v}$ ), where $l_{i}=k_{i}-2$ or $k_{i}$, according to whether or not $J_{k_{i}} \leq Y$;

(ii) $\bar{V} \downarrow v=\sum V\left(2 l_{i}\right)$ (an orthogonal sum, possibly not distinguished).

Proof The summands in $Y$ have the form $J_{k_{i}}$ for $k_{i}>1$. It follows that for each $i$ the fixed space of $u$ on $J_{k_{i}}+J_{k_{i}}{ }^{v}=V\left(2 k_{i}\right)$ is singular, and as $Y+Y^{v}$ is an orthogonal sum of such spaces, $S+S^{v}=C_{Y+Y^{v}}(u)$ is singular in $V$, which means that $S$ is singular in $E$ with respect to $(,)_{v}$. The result now follows from Lemma 4.11. Note that $\bar{V} \downarrow v$ is distinguished if and only there do not exist distinct $i, j, k$ with $l_{i}=l_{j}=l_{k}$, which by (i) may or may not be the case.

Lemma 4.13 Suppose $k$ is odd, $E \downarrow u=J_{k}+J_{k}$ and $V \downarrow v=V(2 k)+V(2 k)$.

(i) If $k=1$, then $u \in C_{G}(v)=C_{G}(v)^{\circ}$, a 1-dimensional unipotent group.

(ii) If $k \geq 3$, then $u \in C_{G}(v)^{\circ}$. Also, $C_{G}(v)=U_{2 k-1} .2$.

Proof (i) Here $G=S L_{2}$, so that $v$ induces an inner automorphism of order 2 (as $v$ is distinguished) and $C_{G}(v)$ is a 1-dimensional unipotent group. For (ii) consider $A_{k-1} A_{k-1}<A_{2 k-1}$. With notation as in (6), we may write $v=\gamma x_{1} x_{2}$, where $x_{i}=u_{i, 1} \ldots u_{i, \frac{k-1}{2}}$ for $i=1,2$. Then $v^{2}=u_{1} u_{2}$ where $u_{1}, u_{2}$ are regular unipotent elements in the corresponding $A_{k-1}$ factors.

There is an element $s \in C_{G}(v)$ such that $u_{1, j}{ }^{s}=u_{2, j}$ for each $j$. Then $u_{1}{ }^{s}=u_{2}$ so that $\left[u_{1}, s\right]=u_{1}{ }^{-1} u_{2} \equiv u \bmod C_{G}(v)^{\circ}$ by Lemma 4.7. Now Lemma 4.8 implies that $u \in C_{G}(v)^{\circ}$.

The dimension of $C_{G}(v)$ is $2 k-1$, by the formulæ (3), (4) in subsection 1.3. Hence to complete the proof it suffices to show that $u_{1} \notin C_{G}(v)^{\circ}$. We proceed by induction. Lemma 4.10 gives the assertion for $k=3$. Assume $k>3$ and consider the parabolic subgroup $P=\operatorname{stab}_{G}\left(C_{E}(u)\right) \cap \operatorname{stab}_{G}\left(C_{E}(u)^{\perp_{v}}\right)$. By Lemmas 4.11 and 4.12, $P=Q L$ is $v$-invariant and $\bar{V} \downarrow v=V(2 k-4)+V(2 k-4)$. Also, $L^{\prime}=A_{1} A_{2 k-5} A_{1}$ and $u Q \in A_{2 k-5}$ has type $J_{k-2}+J_{k-2}$ on the natural module $\bar{E}$ for $A_{2 k-5}$. Inductively, $u_{1} Q \notin C_{A_{2 k-5} Q / Q}(v)^{\circ}$. On the other hand $C_{G}(v)^{\circ} Q / Q<C_{L Q / Q}(v)^{\circ}$, so this gives the assertion.

Lemma 4.14 Suppose $E \downarrow u=J_{k+2}+J_{k}$ with $k$ odd. Then

(i) $u \in C_{G}(v)^{\circ}$;

(ii) if $k>1$ then $x_{1} \notin C_{G}(v)^{\circ}$.

Proof Proceed by induction. The base case $E \downarrow u=J_{3}+J_{1}$ is settled in Lemma 4.10 , so assume $k \geq 3$. Here $C_{G}(u) \leq \hat{P}$ where $\hat{P}$ is determined by the labelled Dynkin diagram 2020 ...202 (see the discussion prior to Lemma 3.4). Then $\hat{P}$ is $\tau$-invariant and Lemma 3.5 shows that this parabolic subgroup is also invariant under the action of $v$. Letting $\hat{Q}=R_{u}(\hat{P})$ we see that $Z(\hat{Q})=U_{\alpha}$, 
a root subgroup. Then $C_{G}\left(U_{\alpha}\right)=P=Q L$, a $v$-invariant parabolic subgroup containing $\hat{P}$, with $L^{\prime}=A_{2 k-1}$. So $u Q=u_{1} u_{2} Q$ has type $J_{k}{ }^{2}$ on the natural module for $A_{2 k-1}$. Then $\operatorname{dim} C_{L^{\prime}}(v Q)=2 k-1$ whereas $\operatorname{dim} C_{G}(v)=2 k$.

Now $Q / Q^{\prime}$ has the structure of the sum of a natural module for $L^{\prime}$ and its dual, with the terms interchanged by $v$. Therefore the fixed space of $u$ has dimension 4 and the fixed space for $v$ has dimension 2. Denote the latter space by $F / Q^{\prime}$.

We claim that $[v, F]=Q^{\prime}$. To see this consider just the factor $A_{k+1} .2$ and the element $\tau u_{1}$. Using a similar argument to that in the first paragraph we obtain a parabolic subgroup $\tilde{P}=\tilde{Q} \tilde{L}$ which is the normalizer of a root subgroup. Then $C_{\tilde{Q} / \tilde{Q}^{\prime}}(v)$ consists of all elements of form $X(c)=x_{11 \ldots 110}(c) x_{011 \ldots 11}(c)$ for $c \in K$ (root elements relative to the $A_{k+1}$ system). As in the proof of Lemma 4.7, $X(c)^{v}=X(c) x_{11 \ldots 11}(c)$. Since $\tilde{Q}<Q$, the claim follows.

By the claim, the map $f \mapsto[v, f]$ from $F$ to $Q^{\prime}$ is surjective, so $C_{F}(v)$ has dimension 2. Moreover $F / Q^{\prime}$ is the direct product of the groups $\langle X(c)$ : $c \in K\rangle Q^{\prime} / Q^{\prime}$ and $C_{F}(v) Q^{\prime} / Q^{\prime}$, so it follows that $C_{F}(v)$ is connected, whence $C_{Q}(v)=U_{2}$, a connected unipotent group of dimension 2 .

Let $X / Q=C_{G}(v)^{\circ} Q / Q$ and $Y / Q=C_{L^{\prime} Q / Q}(v Q)$. It follows that $X / Q$ has codimension 1 in $Y / Q$ and hence is normal. The proof of Lemma 4.13 shows that there is an element $s Q \in C_{L^{\prime} Q / Q}(v Q)$ such that $\left[s Q, u_{1} Q\right]=u Q \in Y / Q$. As the component group of $C_{L^{\prime} Q / Q}(v Q)$ is generated by $x_{1} Q$, we may take $s Q \in Y / Q$. But $Y / X$ is a 1-dimensional unipotent group and hence is centralized by $x_{1} Q$. It follows that $u Q \in X / Q$ so that $u \in C_{G}(v)^{\circ}$, proving (i). Part (ii) now follows using induction and Lemma 4.13.

Lemma 4.15 Suppose $E \downarrow u=J_{k_{1}}+J_{k_{2}}$ with $k_{1}>k_{2}+2$. Then

$$
C_{G}(v) / C_{G}(v)^{\circ} \cong \begin{cases}Z_{2} \times Z_{2} & \text { if } k_{2}>1 \\ Z_{2} & \text { if } k_{2}=1 .\end{cases}
$$

Proof Proceed by induction. If $k_{2}>1$, let

$$
P=\operatorname{stab}_{G}\left(C_{E}(u)\right) \cap \operatorname{stab}_{G}\left(C_{E}(u)^{\perp_{v}}\right),
$$

which by Lemma 4.11 is a $v$-invariant parabolic subgroup. Then $P=Q L$ where $L^{\prime}=A_{1} A_{k_{1}+k_{2}-5} A_{1}$. Lemma 4.12 implies that $u Q$ has type $J_{k_{1}-2}+J_{k_{2}-2}$ on the natural module $\bar{E}$ for $A_{k_{1}+k_{2}-5}$, and $\bar{V} \downarrow v=V\left(2 k_{1}-4\right)+V\left(2 k_{2}-4\right)$. If $k_{2}>3$, then inductively the component group is $Z_{2} \times Z_{2}$, so Lemma 4.8 implies that the same holds for $C$.

Suppose that $k_{2}=3$. Then induction shows that the component group modulo $Q$ is $Z_{2}$, generated by $u_{1} Q$, whereas $u_{2} Q$ is trivial. In particular $u_{1}, u \notin$ $C_{G}(v)^{\circ}$. Let $P_{1}=\operatorname{stab}_{G}(S) \cap \operatorname{stab}_{G}\left(S^{\perp_{v}}\right)$ where $S$ is the fixed space of $u$ on $J_{k_{1}}$. Now consider the quotient $S^{\perp_{v}} / S$ and repeat the process until one arrives at a Levi factor of type $A_{5}$ where the image of $u$ has type $J_{3}+J_{3}$. It follows from Lemma 4.13 that the image of $u_{2}$ is not contained in the connected centralizer 
of the image of $v$. Hence $u_{2} \notin C_{G}(v)^{\circ}$, which together with the above yields the assertion.

So now assume $k_{2}=1$. Here we must show the component group is $Z_{2}$. Let $P_{1}$ be as in the second paragraph. We again obtain the result inductively, provided $k_{1}>5$; otherwise the hypothesis does not hold in the quotient space. So we are reduced to the case $E \downarrow u=J_{5}+J_{1}$, where Lemma 4.10 gives the assertion.

We can now complete the analysis of the component group in the distinguished case, which, in view of Lemma 4.9, is all that is required.

Lemma 4.16 Theorem 1.2 holds if $V \downarrow v=\sum_{k_{i} \text { odd }} V\left(2 k_{i}\right)$.

Proof Here $E \downarrow u=\sum_{i=1}^{r} J_{k_{i}}$, where the $k_{i}$ are odd and in non-increasing order. There is an equivalence relation generated by the condition that $k_{i}$ and $k_{i+1}$ are related (linked) if either $k_{i}=k_{i+1}$ or $k_{i}-k_{i+1}=2$.

Let $C=C_{G}(v)$. Then it follows from Lemmas 4.8 and 4.7 that $C / C^{\circ}$ is generated by commuting elements $s_{i}=u_{i} C^{\circ}$ where $k_{i}>1$. Lemmas 4.13 and 4.14 show that $s_{i} s_{i+1}=1$ if $k_{i}$ and $k_{i+1}$ are linked. Also, if $k_{r}=1$, then any $k_{i}$ linked to 1 satisfies $u_{i} \in C^{\circ}$ by Lemmas 4.14 and 4.10 .

If $C_{i}$ is one of the equivalence classes (linkage classes), then $u_{j} u_{m} \in C^{\circ}$ if $k_{j}, k_{m} \in C_{i}$. So we must show that a product of terms $u_{j}$ is in $C^{\circ}$ if and only if it has the form $c_{1} c_{2} \ldots$, where each $c_{i}$ is a product of an even number of terms $u_{j}$ for $k_{j} \in C_{i}$, or any product of terms $u_{j}$ if $k_{j}$ is linked to $k_{r}=1$.

We show by induction on $\operatorname{dim} V$ that there are no other relations. This will establish Theorem 1.2 for the case where $V \downarrow v$ is distinguished. Suppose $w=\prod u_{i_{j}} \in C^{\circ}$ with $i_{1}>i_{2}>\cdots$ where $w$ is an element of minimal length not of the above form. In particular none of the $u_{i_{j}}$ has the corresponding $k_{i_{j}}$ either equal to or linked to 1 .

First assume $k_{r}>1$. Let $R=C_{E}(u)$ and $P=\operatorname{stab}_{G}(R) \cap \operatorname{stab}_{G}\left(R^{\perp_{v}}\right)$. Then by Lemmas 4.11 and 4.12, $P=Q L$ is a $v$-invariant parabolic subgroup such that $L^{\prime}=A_{r} A_{s} A_{r}$, where $r=\operatorname{dim} R, s=\operatorname{dim}\left(R^{\perp_{v}} / R\right)$ and $u Q \in A_{s} Q$ has type $\sum_{i=1}^{r} J_{k_{i}-2}$.

By induction we obtain a contradiction if there are at least two terms in the product and $k_{i_{2}} \geq 5$. For the exceptional cases first assume there are just two terms and $k_{i_{2}}=3$. Then $w Q=u_{i_{1}} Q$ and by minimality $k_{i_{1}}-2$ is linked to 1 , so that $k_{i_{1}}$ is linked to 3 . That is $k_{i_{1}}$ and $k_{i_{2}}$ are linked, contrary to our hypothesis.

Now suppose $w=u_{k_{i}}$. Here induction gives a contradiction unless $k_{i}-2$ is linked to 1 , that is, $k_{i}$ is linked to 3 . Consequently, we may assume $k_{i}=k_{r}=3$. Let $S=[E, u]^{(5)} \cap C_{E}(u)$ and let $P=\operatorname{stab}_{G}(S) \cap \operatorname{stab}_{G}\left(S^{\perp_{v}}\right)$. This time $u Q$ has the form $\sum_{k_{i}>7} J_{k_{i}-2}+J_{5}{ }^{c}+J_{3}{ }^{d}$ on $\bar{E}$, where $d$ is the multiplicity of $J_{3}$ in $E \downarrow u$ and $c$ is the sum of the multiplicities of $J_{7}$ and $J_{5}$.

If $c \leq 2$ then $v$ is distinguished on $\bar{V}$ and induction gives a contradiction. If 
$c>2$, then

$$
\begin{aligned}
\bar{V} \downarrow v & =\sum_{k_{i}>7} V\left(2 k_{i}-4\right)+V(10)^{c}+V(6)^{d} \\
& =W(10)+\sum_{k_{i}>7} V\left(2 k_{i}-4\right)+V(10)^{c-2}+V(6)^{d} .
\end{aligned}
$$

The image of $w$ comes from the $V(6)^{d}$ summand, within the distinguished part of the sum. The argument at the end of the proof of Lemma 4.9 shows that the component group of the distinguished part lifts faithfully to the full component group. Inductively, we again conclude that $u_{k_{i}} Q$ is not in the connected centralizer of $v$ in $P / Q$.

Finally, we return to the previously excluded case $k_{r}=1$. The above shows that $w$ only involves terms $u_{i_{j}}$ for $k_{i_{j}} \geq 5$. Let $S=[E, u] \cap C_{E}(u)$ and let $P=$ $\operatorname{stab}_{G}(S) \cap \operatorname{stab}_{G}\left(S^{\perp_{v}}\right)$, as in Lemma 4.11. Then $\bar{V}=\sum_{k_{i}>5} V\left(2 k_{i}-4\right)+V(2)^{c}$, where $c$ is the sum of the multiplicities of $V(6)$ and $V(2)$ in the expression for $V \downarrow v$. If $c \leq 2$, then inductively $w Q$ is not in the connected centralizer of $v$ in $P / Q$, so $w \notin C^{\circ}$. And if $c>2$, then $\bar{V}=W(2)+\sum_{k_{i} \geq 5} V\left(2 k_{i}-4\right)+V(2)^{c-2}$. But as $w$ only involves terms $u_{i_{j}}$ for $k_{i_{j}} \geq 5$, it follows that $w Q$ only acts on $\sum_{k_{i} \geq 5} V\left(2 k_{i}-4\right)$. We obtain a contradiction as in the last paragraph.

\subsection{Proof of Theorem 1.3}

Recall that $G=S L_{n}(K)$. As in subsection 1.1, $\sigma$ is a $q$-field morphism commuting with $\tau$, and $\gamma$ is either $\sigma$ or $\sigma \tau$, with $G_{\gamma}=S L_{n}(q)$ or $S U_{n}(q)$ respectively. Again we regard $G\langle\tau\rangle$ as a subgroup of $S p(V)$, where $V=V_{2 n}(K)=E \oplus E^{\tau}$

and $G$ acts on each of the maximal totally singular summands $E$ and $E^{\tau}$. Given $v \in G \tau$, equation (1) in subsection 1.3 states that

$$
V \downarrow v=\sum_{m_{i} \text { odd }} W\left(2 m_{i}\right)^{a_{i}}+\sum_{n_{i} \text { even }} W\left(2 n_{i}\right)^{b_{i}}+\sum_{k_{i} \text { odd }} V\left(2 k_{i}\right) .
$$

We first claim that a representative for the $G$-class of $v$ can be written over the prime field. To see this it will suffice to show that this holds for the individual summands $W(2 m)$ ( $m$ even or odd) and $V(2 k)$ ( $k$ odd).

The proof of Lemma 4.1 shows that $S p_{2 k}(2)$ contains an element $v$ acting on the symplectic module as $V(2 k)$, and for $k$ odd this element interchanges two singular subspaces. This settles the $V(2 k)$ case. Now consider the $W(2 m)$ case. For $m$ odd consider $S p_{2 m}(2) \times S p_{2}(2)$ acting on $V_{2 m} \otimes V_{2}$ and take $v$ to be a regular element in the first factor. Then $v^{2}$ acts as $J_{m}+J_{m}{ }^{\prime}$ on the $V_{2 m}$ space, with $v$ interchanging the blocks. Hence $v$ interchanges the singular spaces $J_{m} \otimes V_{2}$ and $J_{m}{ }^{\prime} \otimes V_{2}$, giving the assertion. Finally, for $m$ even, set $v=\tau u_{1} u_{2} \ldots u_{m-1}$. Then $v^{2}$ has Jordan form $J_{m}{ }^{2}$ on $E$. As an element of $S p(4 m)$ we could have $V \downarrow v=W(2 m)$ or $V(2 m)^{2}$. But since $v \in S L_{2 m} \tau$ the latter is impossible, as is shown by the decomposition (1) repeated above. This 
establishes the claim, from which it follows that $\gamma$ stabilizes each orbit in the action of $G$ on outer unipotent elements in $G \tau$.

Let $v \in S L_{2 m}(2) \tau$ be as above and set $C=C_{G}(v)$. By the above we can choose $\sigma$ and hence $\gamma$ to normalize each of the factors $G_{i}=S L_{2 a_{i} m_{i}}, S L_{2 b_{i} n_{i}}$ and $S L_{k_{i}}$ corresponding to the decomposition (1). Now consider the action of $\gamma$ on $C_{G}(v)$. Using Lemmas 4.4 and 4.7 we see that $\gamma$ acts on the appropriate classical group $C_{G_{i}}(v) / R_{u}\left(C_{G_{i}}(v)\right)$, centralizing the component group. This implies that $\gamma$ leaves invariant each of the factors $S p_{2 a_{i}}$ and $I_{2 b_{i}}$ (even if a group $O_{2 b_{i}}$ pumps up to $\left.S p_{2 b_{i}}\right)$ of $C / R_{u}(C)$ and acts trivially on $C / C^{\circ}$.

At this point we apply the usual Lang-Steinberg theory; we refer the reader to [10] for details. We find that $v^{G} \cap G_{\gamma} \tau$ splits into $2^{s+t+\delta}$ classes and these correspond to representatives $c C^{\circ}$ of $C / C^{\circ}$. For such a representative consider the fixed points of $\gamma c$ (a $G$-conjugate of $\gamma$ ) on $C$. Setting $D=R_{u}(C)=U_{d}$, we see that $\left|D_{\gamma c}\right|=q^{d}$ and $C_{\gamma c}$ covers $(C / D)_{\gamma c}$. Moreover, $\gamma c$ acts on each of the factors $S p_{2 a_{i}}$ or $I_{2 b_{i}}$ as a field or graph field morphism, with all $2^{s}$ possibilities for the fixed points occurring equally often. Theorem 1.3 follows.

\section{Proof of Theorems 1.4 and 1.5}

In this section we assume $(G, p)=\left(E_{6}, 2\right)$ or $\left(D_{4}, 3\right)$ (with $G$ simply connected or adjoint in the latter case); we shall prove Theorems 1.4 and 1.5.

\subsection{Possibilities for the $p$ th power $u$}

We first seek to determine the possible unipotent elements $u$ that can arise as the $p$ th power of an element $v \in G \tau$. At this stage we shall obtain a list of candidate elements; later we shall see which of these possibilities actually occur. For convenience, in this subsection we shall take $G$ to be simply connected.

As mentioned in subsection 1.1, we let $H$ be a simply connected group of type $E_{7}$ or $F_{4}$ according as $(G, p)=\left(E_{6}, 2\right)$ or $\left(D_{4}, 3\right)$; the assumption on the isogeny type of $G$ means that we may regard $G\langle\tau\rangle$ as a subgroup of $H$. Indeed, in the former case, $H$ has a Levi subgroup $E_{6} T_{1}$ with normalizer $\left(E_{6} T_{1}\right) .2$, in which an outer involution induces a graph automorphism of $E_{6}$ and inverts $T_{1}$; in the latter case, the subgroup of $H$ generated by all root subgroups corresponding to long roots is $D_{4}$, with normalizer $D_{4} \cdot S_{3}$.

Recall that for $X=G$ or $H$ we denote by $V_{X}\left(\lambda_{i}\right)$ the restricted irreducible $X$-module with high weight equal to the $i$ th fundamental weight. In particular, $V_{E_{7}}\left(\lambda_{7}\right)$ is the restricted 56-dimensional module for $E_{7}$. For $p=3$, we will denote by $W_{F_{4}}\left(\lambda_{4}\right)$ the 26-dimensional Weyl module for $F_{4}$ with high weight $\lambda_{4}$, which has the 25-dimensional irreducible module $V_{F_{4}}\left(\lambda_{4}\right)$ as a quotient. As before we write $J_{i}$ for a Jordan block of size $i$; we consider the action of $v \in G \tau$ and $u=v^{p} \in G$ on certain modules for $H$ or $G$.

Lemma 5.1 With notation as above, assume $v^{p}=u$. 
(i) If $(G, p)=\left(E_{6}, 2\right)$ and $V_{E_{6}}\left(\lambda_{1}\right) \downarrow u=J_{a_{1}}+\cdots+J_{a_{t}}$, then $V_{E_{7}}\left(\lambda_{7}\right) \downarrow v=$ $J_{2 a_{1}}+\cdots+J_{2 a_{t}}+J_{2}$.

(ii) If $(G, p)=\left(D_{4}, 3\right)$ and $V_{D_{4}}\left(\lambda_{1}\right) \downarrow u=J_{a_{1}}+\cdots+J_{a_{t}}$, then $W_{F_{4}}\left(\lambda_{4}\right) \downarrow v=$ $J_{3 a_{1}}+\cdots+J_{3 a_{t}}+J_{2}$ or $J_{3 a_{1}}+\cdots+J_{3 a_{t}}+J_{1}^{2}$.

Proof (i) We have $V_{E_{7}}\left(\lambda_{7}\right) \downarrow E_{6}=V_{E_{6}}\left(\lambda_{1}\right) \oplus V_{E_{6}}\left(\lambda_{6}\right) \oplus V_{2}$, where $V_{2}$ is a 2-dimensional space on which $E_{6}$ acts trivially. Under the action of the 1dimensional torus $T_{1}$ mentioned above, the space $V_{2}$ decomposes as a sum of two weight spaces for distinct weights. Therefore $\tau$ interchanges the modules $V_{E_{6}}\left(\lambda_{1}\right)$ and $V_{E_{6}}\left(\lambda_{6}\right)$ and also the weight spaces of $T_{1}$ on $V_{2}$.

If $J$ is a Jordan block of $u$ on $V_{E_{6}}\left(\lambda_{1}\right)$, then $J^{v}$ is a Jordan block of $u$ on $V_{E_{6}}\left(\lambda_{6}\right)$ and $J+J^{v}$ is invariant under $v$. Further the fixed space of $v$ on $J+J^{v}$ is 1-dimensional, from which it follows that $J+J^{v}$ is a single Jordan block of $v$. Also, $v$ acts on $V_{2}$ as a single Jordan block. The assertion follows.

(ii) Here $W_{F_{4}}\left(\lambda_{4}\right) \downarrow D_{4}=V_{D_{4}}\left(\lambda_{1}\right) \oplus V_{D_{4}}\left(\lambda_{3}\right) \oplus V_{D_{4}}\left(\lambda_{4}\right) \oplus V_{2}$, where $V_{2}$ is a 2-dimensional space on which $D_{4}$ acts trivially. The above argument gives the assertion, noting the ambiguity for the action of $v$ on $V_{2}$.

In [9], the first author gives the Jordan structure of unipotent elements of $E_{6}$ on $V_{E_{6}}\left(\lambda_{1}\right)$, of $E_{7}$ on $V_{E_{7}}\left(\lambda_{7}\right)$, and of $F_{4}$ on $W_{F_{4}}\left(\lambda_{4}\right)$. Using this together with the known Jordan structure of unipotent elements of $D_{4}$ on $V_{D_{4}}\left(\lambda_{1}\right)$ described in the proof of Lemma 3.2(iii), we may employ Lemma 5.1 to obtain the list of possibilities for the $H$-class containing $v$ and the $G$-class containing $u$. The notation is as in [11].

Lemma 5.2 Tables 1 and 2 list the possibilities for the $H$-class of $v$ and the $G$-class of $u=v^{p}$ which are consistent with the above information on Jordan block sizes.

In the first column of Tables 1 and 2 , for each possible $G$-class we give the corresponding labelled Dynkin diagram $\Delta$; recall from section 3 that $\Delta$ determines the parabolic subgroup $P=Q L$ of $G$, where the simple roots of $L$ are those with label 0 in $\Delta$. Note that each such $P$ is $\tau$-stable; by Lemma 3.5 it follows that each possible $v$ stabilizes the corresponding $P$.

We conclude this subsection by providing, for each possible $G$-class $u^{G}$, a precise expression for a representative $u$ in the form $\prod u_{\beta_{i}}$.

Lemma 5.3 For each of the $G$-classes $u^{G}$ in the second columns of Tables 1 and 2 , an explicit representative $u$ is given in Tables 3 and 4.

Proof In most cases it is clear that the product of unipotent elements given is in the correct class. For example, consider the expression for the elements of type $A_{2} A_{1}{ }^{2}$ or $A_{4}$ in Table 3. Each of these has the form $u=u_{\alpha} u_{\beta} u_{\gamma} u_{\delta}$. The roots $\alpha, \beta, \gamma, \delta$ form a simple system for a root system of type $A_{2} A_{1}{ }^{2}$ or $A_{4}$, respectively. Then $u$ projects to a regular element in the corresponding 
Table 1: Possible classes $u^{G}$ and $v^{H}$ for $G=E_{6}$

\begin{tabular}{|c|c|c|}
\hline$\Delta$ & $u^{G}$ & $v^{H}$ \\
\hline $\begin{array}{c}00000 \\
0\end{array}$ & 1 & $A_{1}^{4},\left(A_{1}^{3}\right)^{\prime \prime}$ \\
\hline $\begin{array}{c}00000 \\
1\end{array}$ & $A_{1}$ & $A_{2} A_{1}^{3}$ \\
\hline $\begin{array}{c}10001 \\
0\end{array}$ & $A_{1}^{2}$ & $A_{3} A_{1}^{2},\left(A_{3} A_{1}\right)^{\prime \prime}$ \\
\hline $\begin{array}{c}00000 \\
2\end{array}$ & $A_{2}$ & $D_{4} A_{1}$ \\
\hline $\begin{array}{c}00100 \\
0\end{array}$ & $A_{1}{ }^{3}$ & $D_{4}\left(a_{1}\right) A_{1}, A_{3} A_{2} A_{1},\left(A_{3} A_{2}\right)_{2}$ \\
\hline $\begin{array}{c}20002 \\
0\end{array}$ & $A_{2}{ }^{2}$ & $A_{5}^{\prime \prime}, D_{6}\left(a_{2}\right), A_{5} A_{1}$ \\
\hline$\frac{20002}{2}$ & $A_{4}$ & $D_{6}$ \\
\hline $\begin{array}{c}01010 \\
0\end{array}$ & $A_{2} A_{1}^{2}$ & $D_{5}\left(a_{1}\right) A_{1}$ \\
\hline $\begin{array}{c}00200 \\
0\end{array}$ & $D_{4}\left(a_{1}\right)$ & $D_{5} A_{1}$ \\
\hline $\begin{array}{c}10101 \\
0\end{array}$ & $A_{2}^{2} A_{1}$ & $E_{7}\left(a_{5}\right)$ \\
\hline${ }_{0}^{20202}$ & $E_{6}\left(a_{3}\right)$ & $E_{7}\left(a_{2}\right)$ \\
\hline${ }_{2}^{22022}$ & $E_{6}\left(a_{1}\right)$ & $E_{7}$ \\
\hline
\end{tabular}

Table 2: Possible classes $u^{G}$ and $v^{H}$ for $G=D_{4}$

\begin{tabular}{|c|l|l|}
\hline$\Delta$ & $u^{G}$ & $v^{H}$ \\
\hline $00_{0}^{0}$ & 1 & $\tilde{A}_{2}, \tilde{A}_{2} A_{1}$ \\
$10_{1}^{1}$ & $A_{1}{ }^{3}$ & $C_{3}, F_{4}\left(a_{2}\right)$ \\
$20_{2}^{2}$ & $D_{4}\left(a_{1}\right)$ & $F_{4}$ \\
\hline
\end{tabular}


Table 3: Class representatives $u$ in $G=E_{6}$

\begin{tabular}{|c|c|}
\hline$u^{G}$ & $u$ \\
\hline 1 & 1 \\
\hline$A_{1}$ & $u_{12321}$ \\
\hline$A_{1}^{2}$ & $u_{12211} u_{11221}$ \\
\hline$A_{2}$ & $u_{01210} u_{11111}$ \\
\hline$A_{1}^{3}$ & $u_{11211} u_{12210} u_{01221}$ \\
\hline$A_{2}{ }^{2}$ & $u_{00111} u_{01111} u_{11100} u_{11110}$ \\
\hline$A_{4}$ & $u_{00011} u_{00110} u_{11100} u_{01100}$ \\
\hline$A_{2} A_{1}^{2}$ & $u_{01110} u_{11111} u_{11210} u_{01211}$ \\
\hline$D_{4}\left(a_{1}\right)$ & $u_{00100} u_{11111} u_{00100} u_{01110}$ \\
\hline$A_{2}^{2} A_{1}$ & $u_{00111} u_{01111} u_{11100} u_{11110} u_{01210}$ \\
\hline$E_{6}\left(a_{3}\right)$ & $u_{11000} u_{00011}^{0} u_{01110}^{010000} u_{0}^{00001} u_{00100}$ \\
\hline$E_{6}\left(a_{1}\right)$ & $u_{00001}^{0} u_{00100} u_{00110} u_{10000} u_{00000} u_{01000}$ \\
\hline
\end{tabular}

Table 4: Class representatives $u$ in $G=D_{4}$

\begin{tabular}{|l|l|}
\hline$u^{G}$ & $u$ \\
\hline 1 & 1 \\
$A_{1}{ }^{3}$ & $u_{11_{0}^{1}} u_{11_{1}^{0}} u_{01}^{1}$ \\
$D_{4}\left(a_{1}\right)$ & $u_{00_{1}^{0}} u_{00_{0}^{1}} u_{01_{0}^{1}} u_{10_{0}^{0}} u_{11_{0}^{0}}$ \\
\hline
\end{tabular}


subsystem subgroup. A similar analysis covers all cases other than $E_{6}\left(a_{1}\right)$, $E_{6}\left(a_{3}\right)$ and $D_{4}\left(a_{1}\right)$. For each of these cases let $u$ be the element given.

Consider $E_{6}\left(a_{1}\right)$. The labelled Dynkin diagram is $\frac{22022}{2}$, so that $u \in Q=$ $Q_{\geq 2}$. To see that $u$ is in the correct class it suffices by parts (ii) and (iii) of Lemma 3.4 to show that $u Q_{>2}$ is in the dense orbit of $L$ on $Q / Q_{>2}$. Now $P$ is a distinguished parabolic subgroup, so $\operatorname{dim} L=\operatorname{dim}\left(Q / Q_{>2}\right)$, and it will suffice to show that the stabilizer in $L$ of $u Q_{>2}$ is finite. The results of [2] imply that $L^{\prime}$ acts on $Q / Q_{>2}$ as on the sum of 2 trivial modules and 3 natural modules. Consider the projections of $u Q_{>2}$ to the modules $\left\langle U_{0}^{01000} U_{01100}\right\rangle Q_{>2}$ and $\left\langle U_{00100} U_{00110}\right\rangle Q_{>2}$. The projections are, respectively, minimal and maximal vectors of these natural modules. As $C_{L}\left(u Q_{>2}\right)$ must stabilize each of these projections we conclude that $C_{L}\left(u Q_{>2}\right) \leq T_{G}$, a maximal torus of $L$. But as the roots appearing in $u$ and their negatives generate the full root system of $G$, we conclude that $C_{L}\left(u Q_{>2}\right)=1$.

Similar but easier considerations apply to $D_{4}\left(a_{1}\right)$ in Table 3 . Start with the subsystem subgroup of type $D_{4}$ with simple system $\stackrel{11111}{0}, \underset{1}{00000}, \underset{0}{00100}, \underset{0}{01110}$. Then $u$ is contained in the unipotent radical of the parabolic subgroup determined by the labelled Dynkin diagram 202 , and as above we see that $u$ is distinguished in this $D_{4}$. Now $D_{4}$ has two conjugacy classes of distinguished unipotent elements, namely the regular elements and those acting on the usual orthogonal module as the sum of two orthogonal Jordan blocks of size 4. Clearly $u$ is not a regular element since it lies in all Borel subgroups of the parabolic subgroup indicated. So $u$ has type $D_{4}\left(a_{1}\right)$. The case of the class $D_{4}\left(a_{1}\right)$ in Table 4 is similar.

Now consider $E_{6}\left(a_{3}\right)$. As noted in the proof of Lemma 3.2(ii), elements of this type are represented in $F_{4}$ as unipotent elements of type $F_{4}\left(a_{2}\right)$. Also $u^{\tau}=u$, so $u \in F_{4}$. With $u=\prod u_{\beta_{i}}$, set $e=\sum e_{\beta_{i}}$. Then from the $F_{4}\left(a_{2}\right)$ nilpotent element of [11, Table 13.3] and the usual folding of the root system, we see that in the Lie algebra $L\left(F_{4}\right)$ the nilpotent element $e$ is distinguished of type $F_{4}\left(a_{2}\right)$. Now [11, Lemma 19.7] shows that $u$ is distinguished of type $F_{4}\left(a_{2}\right)$ in $F_{4}$ and hence is a distinguished unipotent element of type $E_{6}\left(a_{3}\right)$ in $G$.

\subsection{The elements $v_{i}$}

In this subsection we shall consider the elements $v_{i}$ listed in Tables 7 and 8 , and begin the process of showing that the information on each element presented there is correct. We continue to assume $G$ is simply connected, so that $G\langle\tau\rangle<H$ where $H$ is simply connected of type $E_{7}$ or $F_{4}$.

We will require the following standard notation. Recall that in the root system $\Sigma$ we have the simple system $\Pi=\left\{\alpha_{1}, \ldots, \alpha_{r}\right\}$. For $j=1, \ldots, r$ and $c \in K^{*}$, let $h_{j}(c)$ denote the usual element of $\left\langle X_{\alpha_{j}}, X_{-\alpha_{j}}\right\rangle \cap T_{G}$ such that $x_{\alpha_{j}}(t)^{h_{j}(c)}=x_{\alpha_{j}}\left(c^{2} t\right)$ for all $t \in K$. Explicit expressions for these elements are given in [3, Lemma 6.4.4], although adjustments must be made to account for 
the fact that here we are acting on the right rather than the left.

It will also be convenient to use a certain abbreviated notation. As examples set

$$
\begin{aligned}
x_{3}(c) & =x_{01000}(c), \\
x_{245}(c) & =x_{00110}(c), \\
x_{3,5}(c) & =x_{01000}(c) x_{00010}(c), \\
x_{1,4,6}(c) & =\underset{0}{10000}(c) \underset{0}{x_{00100}}(c) x_{0}^{00001}(c),
\end{aligned}
$$

etc. Similarly, set

$$
\begin{aligned}
Y_{2} & =\left\langle x_{2}(c), x_{-2}(c)\right\rangle, \\
Y_{2,3,5} & =\left\langle x_{2}(c) x_{3}(c) x_{5}(c), x_{-2}(c) x_{-3}(c) x_{-5}(c)\right\rangle, \\
Y_{13,56} & =\left\langle x_{13,56}(c), x_{-13,-56}(c)\right\rangle,
\end{aligned}
$$

etc.

Finally, we give $\tau$ explicitly as an element of $H$. For $\beta$ a root of $H$ we write $s_{\beta}$ for the standard representative of the Weyl group reflection in $\beta$. According as $H=E_{7}$ or $F_{4}$ we take

$$
\tau=s_{122111} s_{112211} s_{012221} \quad \text { or } \quad \tau=s_{0001} s_{0010} .
$$

In the former case this suffices to determine the action of $\tau$ on $G$. In the latter case, however, in order to distinguish $\tau$ from its inverse we must specify the correspondence between roots of $G$ and of $H$. We take $100000{ }_{0}^{0}, 0{ }_{0}^{1}$ and 000 to be 0100, 1000, 0120 and 0122 respectively; thus with $\tau$ as above we have

$$
X_{10_{0}^{0}}^{\tau}=X_{00}^{1}{ }_{0}^{1}, \quad X_{00}^{1}{ }_{0}^{\tau}=X_{00}^{0}, \quad X_{00{ }_{1}^{0}}^{\tau}=X_{10_{0}^{0}} .
$$

Our first result here is then the following.

Lemma 5.4 For each element $v_{i}$ listed in the second column of Table 7 or 8, its pth power $v_{i}{ }^{p}$ is the element $u$ listed in Table 3 or 4 for the corresponding G-class.

Proof This is simply a direct check.

We next determine the $H$-class of each element $v_{i}$.

Lemma 5.5 (i) For $G=E_{6}$ and $i=1, \ldots, 17$, the $E_{7}$-class of the element $v_{i}$ is as indicated in the third column of Table 7.

(ii) For $G=D_{4}$ and $i=1, \ldots, 5$, the $F_{4}$-class of the element $v_{i}$ is as indicated in the third column of Table 8. 
Proof (i) A computer calculation determines the Jordan forms of the elements $v_{i}$ on both $V_{E_{7}}\left(\lambda_{7}\right)$ and $L\left(E_{7}\right)$. At this point, the results in [9] suffice to identify the class of $v_{i}$, with the exceptions of $v_{10}$ and $v_{11}$. The Jordan form information shows that these particular elements must have type $A_{5} A_{1}$ or $D_{6}\left(a_{2}\right)$, but these classes are not distinguished by their Jordan form on either $V_{E_{7}}\left(\lambda_{7}\right)$ or $L\left(E_{7}\right)$. However, it follows that each of $v_{10}$ and $v_{11}$ is centralized by a 1-dimensional torus, say $T_{1}$, of $E_{7}$ and is a distinguished unipotent element in the semisimple part of $C_{E_{7}}\left(T_{1}\right)$.

Now $v_{11}$ is centralized by the 1-dimensional torus $S=\left\{h_{4}(c): c \in K^{*}\right\}$. Then $S$ lies in a fundamental $A_{1}$ subgroup, and it follows that $C_{E_{7}}(S)=D_{6} S$. Therefore $S$ is conjugate to $T_{1}$ and $v_{11}$ is distinguished in $D_{6}$, and hence has type $D_{6}\left(a_{2}\right)$ by the above.

Next $v_{10}$ is centralized by the 1-dimensional torus $S=\left\{h_{2}(c) h_{3}(c) h_{5}(c)\right.$ : $\left.c \in K^{*}\right\}$. One checks that $S$ centralizes the subsystem subgroup of type $A_{5} A_{1}$, where the $A_{5}$ has simple system ${ }_{1}^{111000},{ }_{0}^{011110}, 000001,{ }_{1}^{001110},{ }_{0}^{11100}$ and the $A_{1}$ has simple system ${ }_{1}^{012100}$. It follows that $v_{10}$ has type $A_{5} A_{1}$ in $E_{7}$.

(ii) As above, the result follows from calculating the Jordan forms of the elements $v_{i}$ on both $L\left(F_{4}\right)$ and $W_{F_{4}}\left(\lambda_{4}\right)$ and applying the results of [9].

\subsection{The centralizers $C_{G}\left(v_{i}\right)$}

By now the only entries in Tables 7 and 8 which we must establish are those in the final column, giving the centralizers $C_{G}\left(v_{i}\right)$. Recall that by the final paragraph of subsection 1.1 these are independent of the isogeny type of $G$; we shall in fact assume $G$ is adjoint in this subsection.

In the lemmas to follow we shall make frequent use of two pieces of information for a given element $v_{i}$ with $p$ th power $u$. Firstly, the structure of $C_{P}(u) / C_{Q}(u)$ is given in Table 22.1.3 or Table 8.5a of [11] according as $G=E_{6}$ or $D_{4}$. Secondly, Tables 5 and 6 give for each $v_{i}$ the structure of $C_{Q}\left(v_{i}\right)$ and $C_{U}\left(v_{i}\right)$ (recall that $U=\prod_{\beta \in \Sigma^{+}} X_{\beta}$, where $\Sigma^{+}$is the positive system determined by $\Pi$ ). The information in these tables summarizes results obtained by performing computer calculations to identify the $U$-centralizers explicitly; these results are presented in more detailed form in the appendix. For convenience of reference, the first column of Tables 5 and 6 gives the labelled Dynkin diagram $\Delta$, which determines the parabolic subgroup $P$, while the second column repeats the definition of the element $v_{i}$.

In the lemmas which follow, we treat together elements $v_{i}$ having the same $p$ th power. We begin with $G=E_{6}$.

Lemma 5.6 If $G=E_{6}$ then $C_{G}\left(v_{i}\right)$ is as given in Table 7 for $i=1,2$.

Proof This is well known: it is shown in $[1,19.9]$ that $C_{G}\left(v_{1}\right) \cong F_{4}$ and $C_{G}\left(v_{2}\right)$ is isomorphic to the centralizer in $F_{4}$ of a long root element.

Lemma 5.7 If $G=E_{6}$ then $C_{G}\left(v_{i}\right)$ is as given in Table 7 for $i=3$. 
Table 5: $C_{Q}\left(v_{i}\right)$ and $C_{U}\left(v_{i}\right)$ for $G=E_{6}$

\begin{tabular}{|c|c|c|c|}
\hline$\Delta$ & $v_{i}$ & $C_{Q}\left(v_{i}\right)$ & $C_{U}\left(v_{i}\right)$ \\
\hline \multirow[t]{2}{*}{$\begin{array}{c}00000 \\
0\end{array}$} & $v_{1}=\tau$ & 1 & $U_{24}$ \\
\hline & $v_{2}=\tau u_{12321}$ & 1 & $U_{24}$ \\
\hline $\begin{array}{c}00000 \\
1\end{array}$ & $v_{3}=\tau u_{01210} u_{11111}$ & $U_{14}$ & $U_{20}$ \\
\hline \multirow[t]{3}{*}{$\begin{array}{c}10001 \\
0\end{array}$} & $v_{4}=\tau u_{12211}$ & $U_{15}$ & $U_{21}$ \\
\hline & $v_{5}=\tau u_{01210} u_{12211}$ & $U_{11}$ & $U_{20}$ \\
\hline & $v_{6}=\tau u_{01100} u_{00110} u_{01210} u_{11111} u_{12211}$ & $U_{11}$ & $U_{20}$ \\
\hline$\frac{00000}{2}$ & $v_{7}=\tau s_{1} s_{6} s_{4} u_{11111}$ & $U_{10}$ & $U_{12}$ \\
\hline $\begin{array}{c}00100 \\
0\end{array}$ & $v_{8}=\tau u_{00100} u_{11111} u_{12210}$ & $U_{15}$ & $U_{16}$ \\
\hline \multirow[t]{3}{*}{$\begin{array}{c}20002 \\
0\end{array}$} & $v_{9}=\tau u_{11100} u_{11110}$ & & $U_{14}$ \\
\hline & $v_{10}=\tau u_{11100} u_{11110} u_{01210}$ & $U_{8}$ & $U_{14}$ \\
\hline & $v_{11}=\tau u_{0}^{01110} u_{01100}^{0100110} u_{11100} u_{11110}$ & $U_{8} .2$ & $U_{12.2}$ \\
\hline \multirow[t]{2}{*}{$\begin{array}{c}20002 \\
2\end{array}$} & $v_{12}=\tau s_{4} u_{11100} u_{01100}$ & $U_{7} .2$ & $U_{8} .2$ \\
\hline & $v_{13}=\tau s_{4} u_{11100} u_{01100} u_{01110}$ & $U_{7}$ & $U_{8}$ \\
\hline $\begin{array}{c}00200 \\
0\end{array}$ & $v_{14}=\tau s_{1} s_{6} s_{2} u_{00100} u_{01110}$ & $U_{9}$ & $U_{9}$ \\
\hline $\begin{array}{c}10101 \\
0\end{array}$ & $v_{15}=\tau u_{11100} u_{11110} u_{01110} u_{00100}$ & $U_{12.4}$ & $U_{12.4}$ \\
\hline $\begin{array}{c}20202 \\
0\end{array}$ & $v_{16}=\tau s_{2} s_{3} s_{5} u_{10000} u_{00001} u_{00100}$ & $U_{6}$ & $U_{6}$ \\
\hline$\frac{22022}{2}$ & $v_{17}=\tau s_{4} u_{0} \underset{0}{1000} u_{0} 0000 u_{01000}$ & $U_{4} .2$ & $U_{4} .2$ \\
\hline
\end{tabular}

Table 6: $C_{Q}\left(v_{i}\right)$ and $C_{U}\left(v_{i}\right)$ for $G=D_{4}$

\begin{tabular}{|c|l|l|l|}
\hline$\Delta$ & $v_{i}$ & $C_{Q}\left(v_{i}\right)$ & $C_{U}\left(v_{i}\right)$ \\
\hline $00_{0}^{0}$ & $v_{1}=\tau$ & 1 & $U_{6}$ \\
& $v_{2}=\tau u_{12}{ }_{1}^{1}$ & 1 & $U_{6}$ \\
\hline 101 & $v_{3}=\tau u_{11_{0}^{1}}$ & $U_{3}$ & $U_{4}$ \\
& $v_{4}=\tau u_{11_{0}^{1}} u_{01_{0}^{0}}$ & $U_{3}$ & $U_{4} \cdot 3$ \\
\hline 202 & $v_{5}=\tau u_{10_{0}^{0}} u_{01_{0}^{0}}$ & $U_{2}$ & $U_{2}$ \\
\hline
\end{tabular}


Proof Since $v_{3} Q / Q=\tau Q / Q$, and $C_{P}\left(v_{3}\right) \leq C_{P}(u)=Q A_{5}$ by [11, Table 22.1.3], it follows that $C_{P}\left(v_{3}\right) Q / Q \leq C_{3} Q / Q$ where the group $C_{3}$ consists of the fixed points of $\tau$ on the $A_{5}$ Levi subgroup. If we write $\delta_{1}, \delta_{2}, \delta_{3}$ for the simple roots of this $C_{3}$, then for $c \in K$ we have $x_{\delta_{1}}(c)=x_{1}(c) x_{6}(c), x_{\delta_{2}}(c)=x_{3}(c) x_{5}(c)$ and $x_{\delta_{3}}(c)=x_{4}(c)$. We now produce a subgroup $G_{2}$ in this $C_{3}$.

Start with a group $B_{3}$ defined over $K$, with simple roots $\beta_{1}, \beta_{2}, \beta_{3}$ (numbered in the usual manner). By taking the fixed points of a triality automorphism of $D_{4}$, we see that there is a group $G_{2}<B_{3}$, with simple roots $a$ (short) and $b$ (long), generated by root elements $x_{a}(c)=x_{\beta_{1}}(c) x_{\beta_{3}}(c)$ and $x_{b}(c)=$ $x_{\beta_{2}}(c)$ for $c \in K$ along with corresponding elements for negative roots. Since $p=2$, we have a surjection $B_{3} \rightarrow C_{3}$ with $x_{\beta_{1}}(c) \mapsto x_{\delta_{1}}(c), x_{\beta_{2}}(c) \mapsto x_{\delta_{2}}(c)$, $x_{\beta_{3}}(c) \mapsto x_{\delta_{3}}\left(c^{2}\right)$. Thus we have $G_{2}<C_{3}$ generated by elements $x_{\delta_{1}}(c) x_{\delta_{3}}\left(c^{2}\right)=$ $x_{1}(c) x_{6}(c) x_{4}\left(c^{2}\right)$ and $x_{\delta_{2}}(c)=x_{3}(c) x_{5}(c)$ for $c \in K$ together with negatives.

Now by inspection of the detailed information given in the appendix for $C_{U}\left(v_{3}\right)$, we see that $C_{P}\left(v_{3}\right)$ covers the maximal unipotent subgroup of this $G_{2}$. The Weyl group of the $G_{2}$ is generated by the involutions $s_{1} s_{6} s_{4}$ and $s_{3} s_{5}$ of $A_{5}$. One checks that $s_{3} s_{5}$ and $s_{1} s_{6} s_{4} u_{01210}$ centralize $v_{3}$, so it follows that $C_{P}\left(v_{3}\right) Q / Q$ contains a subgroup isomorphic to $G_{2}$. Now $G_{2}$ is a maximal subgroup of $C_{3}$, so if the containment were proper, we would have $C_{P}\left(v_{3}\right) Q / Q \cong$ $C_{3}$, contrary to Table 5 which states that $C_{Q}\left(v_{3}\right)=U_{14}$ while $C_{U}\left(v_{3}\right)=U_{20}$. Therefore $C_{P}\left(v_{3}\right) Q / Q \cong G_{2}$, and thus $C_{P}\left(v_{3}\right)=U_{14} G_{2}$ as required.

Lemma 5.8 If $G=E_{6}$ then $C_{G}\left(v_{i}\right)$ is as given in Table 7 for $i=4,5,6$.

Proof Here $Q_{\geq 2}=Z(Q)$ affords a natural orthogonal module for $L^{\prime}=D_{4}$, with $u \in Q \geq 2$ a non-singular vector. Then $C_{P}(u)<Q B_{3} T_{1}$ by [11, Table 22.1.3], where $B_{3}=C_{L^{\prime}}(\tau)$ and $T_{1}=\left\{h_{1}\left(c^{2}\right) h_{3}(c) h_{5}\left(c^{-1}\right) h_{6}\left(c^{-2}\right): c \in K^{*}\right\}$. We note that $T_{1}$ acts trivially on the orthogonal module and is inverted by $\tau$.

First consider $v_{4}=\tau u_{12211}$. Now $B_{3}$ contains a subgroup $O_{6}=D_{3} .2=$ $\left\langle Y_{4}, Y_{2}, Y_{345}\right\rangle\left\langle s_{3} s_{5}\right\rangle$, where the $D_{3}$ centralizes the non-degenerate 2-space of $Z(Q)$ spanned by the root elements $u_{12211}$ and $u_{11221}$, while $s_{3} s_{5}$ interchanges the basis elements. Also, as $s_{3} s_{5} u_{12211}$ centralizes $v_{4}$ we have $C_{P}\left(v_{3}\right) / C_{Q}\left(v_{3}\right) \geq$ $D_{3} .2$. As $O_{6}$ is a maximal subgroup of $B_{3}$, Table 5 implies that this containment must be an equality, which yields the result for $v_{4}$.

Now consider $v_{5}$ and $v_{6}$. Modulo $Q$ these elements have the form $v_{4} x$ and $v_{4} y$, where $x$ is a long root element of $B_{3}$ for the highest root, and $y$ is the product of $x$ and a short root element for the highest short root. Both $x$ and $y$ are central in the standard maximal unipotent subgroup, and it follows (see for example [11, Lemma 2.4]) that $C_{B_{3}}(x)$ and $C_{B_{3}}(y)$ contain derived groups of parabolic subgroups of $B_{3}$. Checking fundamental reflections we see that $C_{B_{3}}(x)=U_{7} A_{1} A_{1}$ while $C_{B_{3}}(y)=U_{8} A_{1}$, where in the second case the $A_{1}$ corresponds to the fundamental short root of $B_{3}$.

Now $C_{P}\left(v_{i}\right) Q / Q$ is contained in $C_{B_{3}}(x) Q / Q$ or $C_{B_{3}}(y) Q / Q$, respectively, 
and the information on $v_{5}$ and $v_{6}$ in Table 5 shows that $C_{P}\left(v_{i}\right) Q / Q$ contains $\tilde{U} Q / Q$, where $\tilde{U}$ is the standard maximal unipotent subgroup of $B_{3}$. We see that $C_{P}\left(v_{5}\right)$ contains $s_{2}$ and $s_{3} s_{5} u_{12211}$, whereas $C_{P}\left(v_{6}\right)$ contains $s_{3} s_{5}$. It follows that $C_{P}\left(v_{5}\right) Q / Q$ covers $C_{B_{3}}(x)$ while $C_{P}\left(v_{6}\right) Q / Q$ covers $C_{B_{3}}(y)$. These are the derived groups of the standard parabolic subgroups with Levi subgroups $Y_{2} \times Y_{3,5}$ and $Y_{3,5}$, respectively. The result follows.

Lemma 5.9 If $G=E_{6}$ then $C_{G}\left(v_{i}\right)$ is as given in Table 7 for $i=7$.

Proof Here $C_{P}(u) Q / Q=A_{2} A_{2} .2$ by [11, Table 22.1.3], where $A_{2} A_{2}=$ $\left\langle Y_{3}, Y_{456}\right\rangle\left\langle Y_{5}, Y_{134}\right\rangle$ with the factors interchanged by $s_{1} s_{6} s_{4}$. The factors are also interchanged by $\tau$, so that $v_{7} Q=\tau s_{1} s_{6} s_{4} Q$ acts as a graph automorphism on each $A_{2}$ factor of $A_{2} A_{2} Q / Q$. It follows that $C_{P}\left(v_{7}\right) Q / Q \leq A_{1} A_{1} .2$. Moreover, $Y_{3456} \times Y_{1345} \cong A_{1} A_{1}$ centralizes $v_{7}$, as does $s_{1} s_{6} s_{4} u_{01210}$. Therefore, $C_{P}\left(v_{7}\right) Q / Q \cong A_{1} A_{1} .2$ and the conclusion follows using Table 5 .

Lemma 5.10 If $G=E_{6}$ then $C_{G}\left(v_{i}\right)$ is as given in Table 7 for $i=8$.

Proof Here $C_{P}(u) Q=A_{2} A_{1} Q$ by [11, Table 22.1.3] where $A_{1}=Y_{2}$ and $A_{2}=$ $\left\langle Y_{3,6}, Y_{1,5}\right\rangle$. Now $v_{8} Q=\tau Q$ induces a graph automorphism on the $A_{2}$ factor, so that $C_{P}\left(v_{8}\right) Q / Q \leq A_{1} A_{1}$, where $A_{1} A_{1}=Y_{2} \times Y_{13,56}$. The detailed information in the appendix shows that $C_{P}\left(v_{8}\right) Q$ contains the elements $x_{13}(c) x_{56}(c) x_{2}\left(c^{2}\right) Q$ for $c \in K$. Also, $v_{8}$ is centralized by $s_{1}{ }^{s_{3}} s_{6}{ }^{s_{5}} s_{2} u_{00100} u_{01221}$. Therefore, $C_{P}\left(v_{8}\right) Q / Q$ contains a diagonal $A_{1}$ in $A_{1} A_{1}$. By Table 5 we must then have $C_{P}\left(v_{8}\right) Q / Q=A_{1}$, and the result follows.

Lemma 5.11 If $G=E_{6}$ then $C_{G}\left(v_{i}\right)$ is as given in Table 7 for $i=9,10,11$.

Proof Here $u=u_{00111} u_{01111} u_{11100} u_{11110}$. We have $C_{P}(u) Q / Q=G_{2}$ by [11, Table 22.1.3], and indeed $G_{2}=\left\langle Y_{4}, Y_{2,3,5}\right\rangle$ centralizes $u$. As this group also centralizes $v_{9}$, we obtain $C_{P}\left(v_{9}\right)=U_{8} G_{2}$ from Table 5 .

Now consider $v_{10}$ and $v_{11}$. These elements have the form $v_{9} x$ and $v_{9} y$, where $x$ is a long root element of $G_{2}$ for the highest root, and $y$ is a short root element for the highest short root. It follows that $C_{P}\left(v_{i}\right) Q / Q$ is contained in $C_{G_{2}}\left(v_{i}\right) Q / Q=U_{5} A_{1}$ or $U_{3} A_{1}$, respectively. Here the $A_{1}$ factor is just $Y_{2,3,5}$ or $Y_{4}$ according as $i=10$ or 11 . Using the information in the appendix for these elements we see that $C_{P}\left(v_{i}\right) Q / Q=U_{6}$ or $U_{4}$ respectively. One checks that $C_{P}\left(v_{10}\right)$ contains $s_{2} s_{3} s_{5}$ while $C_{P}\left(v_{11}\right)$ contains $s_{4}$. It follows that $C_{P}\left(v_{i}\right) Q / Q=$ $U_{5} A_{1}$ or $U_{3} A_{1}$ respectively.

Another appeal to Table 5 shows that $C_{Q}\left(v_{10}\right)=U_{8}$, whereas $C_{Q}\left(v_{11}\right)=$ $U_{8} .2$. This completes the analysis of $C_{G}\left(v_{10}\right)=C_{P}\left(v_{10}\right)$, but for $v_{11}$ we must verify that $C_{P}\left(v_{11}\right)=U_{11} A_{1} .2$. That is, we must verify that the component group of the centralizer is non-trivial. From the description of $C_{U}\left(v_{11}\right)$ it is 
clear that this group is disconnected with component group of order 2. Also the element $s_{4}$ centralizes this component group. It follows that $C_{P}\left(v_{11}\right)=U_{11} A_{1} .2$, completing the proof.

Lemma 5.12 If $G=E_{6}$ then $C_{G}\left(v_{i}\right)$ is as given in Table 7 for $i=12,13$.

Proof Here we have $u=u_{00011} u_{00110} u_{11100} u_{01100}, v_{12}=\tau s_{4} u_{11100} u_{01100}$, and $v_{13}=v_{12} u_{01110}$. Further $C_{P}(u) Q / Q=A_{1} T_{1}$ by [11, Table 22.1.3], where $A_{1}=Y_{345}$ and $T_{1}=\left\{h_{1}\left(c^{-2}\right) h_{3}\left(c^{-1}\right) h_{4}\left(c^{3}\right) h_{5}(c) h_{6}\left(c^{2}\right): c \in K^{*}\right\}$. Note that $\tau s_{4}$ inverts $T_{1}$.

Now $v_{i} Q=\tau s_{4} Q$ or $\tau s_{4} u_{01110} Q$, according as $i=12$ or $i=13$. As $\tau s_{4}$ centralizes $A_{1}$, we have $C_{P}\left(v_{12}\right) Q / Q \leq A_{1} Q / Q$ and $C_{P}\left(v_{13}\right) Q / Q \leq X_{01110} Q / Q$. By inspection we have $A_{1} \leq C_{P}\left(v_{12}\right)$ and $X_{01110} \leq C_{P}\left(v_{13}\right)$. Therefore, the containments are equalities.

The result now follows from Table 5, except that we must determine the component group of $C_{P}\left(v_{12}\right)$. However, we have seen that this group is the semidirect product of $C_{Q}\left(v_{12}\right)$ and $A_{1}$, and this implies that the component group of $C_{P}\left(v_{12}\right)$ is just that of $C_{U}\left(v_{12}\right)$, which has order 2 .

Lemma 5.13 If $G=E_{6}$ then $C_{G}\left(v_{i}\right)$ is as given in Table 7 for $i=14$.

Proof Here $u=u_{00100} u_{11111} u_{00100} u_{01110}, v_{14}=\tau s_{1} s_{6} s_{2} u_{00100} u_{01110}$, and $C_{P}(u) Q / Q=T_{2} \cdot S_{3}$ by [11, Table 22.1.3]. In this instance we have $T_{2}=$ $\left\{h_{1}(a) h_{3}(b) h_{5}\left(b^{-1}\right) h_{6}\left(a^{-1}\right): a, b \in K^{*}\right\}$; this is inverted by $\tau$ and $C_{T_{2}}\left(\tau s_{1} s_{6} s_{2}\right)$ is the 1-dimensional torus $T_{1}=\left\{h_{1}(c) h_{6}\left(c^{-1}\right): c \in K^{*}\right\}$. Also, $\tau$ centralizes $u$ and [11, Table 22.1.4] implies that $\tau$ centralizes the $S_{3}$ quotient of $C_{P}(u) Q / Q$. It follows that $C_{P / Q}\left(v_{14} Q\right)=\left\langle s_{1} s_{6} s_{2}\right\rangle T_{1} Q / Q$. One checks that $T_{1}$ centralizes $v_{14}$. Also $\tau$ centralizes $v_{14}$, and therefore so does $\tau v_{14} \in s_{1} s_{6} s_{2} Q$. Thus $C_{P}\left(v_{14} Q\right) / Q=\left\langle s_{1} s_{6} s_{2}\right\rangle T_{1} Q / Q$, and the result follows from Table 5 .

Lemma 5.14 If $G=E_{6}$ then $C_{G}\left(v_{i}\right)$ is as given in Table 7 for $i=15$.

Proof Here $u$ has type $A_{2}^{2} A_{1}$ and $C_{P}(u) / C_{Q}(u)=A_{1}$ by [11, Table 22.1.3]. Consider the group $A=Y_{2,3,5}$, which is of type $A_{1}$. Write $h(c)=h_{2}(c) h_{3}(c) h_{5}(c)$ for $c \in K^{*}$, and set $T_{1}=\left\{h(c): c \in K^{*}\right\}$; then $T_{1}$ is a 1-dimensional torus of $A$. Take $\omega \in K^{*}$ with $\omega^{3}=1 \neq \omega$. One checks that $h(\omega)$ and $s=s_{2} s_{3} s_{5} u_{00100}$ centralize $v_{15}$. Modulo $Q$ these elements generate a group of type $S_{3}$. Therefore, $C_{P}\left(v_{15}\right) Q / Q$ contains $S_{3}$.

We claim that $C_{P}\left(v_{15}\right) Q / Q=S_{3}$. To see this view $A$ as a short root $A_{1}$ in the group $G_{2}<D_{4}$, where the $D_{4}$ has simple system ${ }_{0}^{01000}, \underset{0}{00100}, \underset{1}{00000}, \underset{0}{00010}$ and $G_{2}$ is the group of fixed points under the standard triality automorphism. Consider the standard parabolic subgroup $\tilde{P}=\tilde{Q} \tilde{L}>P$, where $\tilde{L}^{\prime}$ is this group 
$D_{4}$. Then $v_{15} \tilde{Q} / \tilde{Q}=\tau u_{01110} u_{00100} \tilde{Q} / \tilde{Q}$ and we view this as contained in $\langle\tau\rangle \times G_{2}$. Now $u_{01110} u_{00100} \tilde{Q} / \tilde{Q}$ is a unipotent element of type $A_{2}$ in $D_{4}$, hence of type $G_{2}\left(a_{1}\right)$ in $G_{2}$. It follows from [11, Table 22.1.5] that the reductive part of the centralizer for this unipotent element is $S_{3}$. This establishes the claim.

The appendix contains a precise description of the elements in $C_{U}\left(v_{15}\right)$. We conclude from this information that the component group of $C_{Q}\left(v_{15}\right)$ is isomorphic to $\mathbb{F}_{4}$ and that $h(\omega)$ acts non-trivially on this component group. Moreover, we see that $x_{2345}(c) x_{4}(c) \in C_{Q}\left(v_{15}\right)$ for all $c \in K$, which implies that $s^{2}=u_{01110} u_{00100} \in C_{Q}\left(v_{15}\right)^{\circ}$. It now follows that the component group of $C_{P}\left(v_{15}\right)$ is isomorphic to $S_{4}$, and so from Table 5 we have $C_{P}\left(v_{15}\right)=U_{12} . S_{4}$ as required.

Lemma 5.15 If $G=E_{6}$ then $C_{G}\left(v_{i}\right)$ is as given in Table 7 for $i=16$.

Proof Here $u$ is a distinguished unipotent element of $G$ and $C_{P}(u) Q / Q=Z_{2}$ by [11, Table 22.1.3]. As $\tau$ centralizes $v_{16}$, so does $\tau v_{16} \in s_{2} s_{3} s_{5} Q$. It follows that $C_{P}\left(v_{16}\right) Q / Q=Z_{2}$, so Table 5 gives $C_{P}\left(v_{16}\right)=U_{6} .2$ as required.

Lemma 5.16 If $G=E_{6}$ then $C_{G}\left(v_{i}\right)$ is as given in Table 7 for $i=17$.

Proof Here $u$ is a distinguished unipotent element of $G$ and $C_{P}(u) Q / Q=1$ by [11, Table 22.1.3]; thus Table 5 gives $C_{P}\left(v_{17}\right)=C_{Q}\left(v_{17}\right)=U_{4} .2$ as required.

Finally we turn to $G=D_{4}$.

Lemma 5.17 If $G=D_{4}$ then $C_{G}\left(v_{i}\right)$ is as given in Table 8 for $i=1,2$.

Proof This is well known: [7, Proposition 4.9.2] shows that $C_{G}\left(v_{1}\right)=G_{2}$ and $C_{G}\left(v_{2}\right)$ is the centralizer of a long root element in $G_{2}$.

Lemma 5.18 If $G=D_{4}$ then $C_{G}\left(v_{i}\right)$ is as given in Table 8 for $i=3,4$.

Proof Here $u=u_{11}^{1}{ }_{0} u_{11_{1}^{0}} u_{01}^{1}{ }_{1}^{1}$ has type $A_{1}{ }^{3}$, and $C_{P}(u) / C_{Q}(u)=A_{1}$ by $[11$, Table 8.5a], where $A_{1}=Y_{2}$. Table 6 shows that $C_{U}\left(v_{3}\right)=U_{4}$ and $C_{U}\left(v_{4}\right)=$ $U_{4} .3$. Since $Y_{2}$ centralizes $v_{3}$, we have $C_{P}\left(v_{3}\right)=U_{3} A_{1}$ as required. Now consider $v_{4}$. Since $v_{4} Q=\tau u_{01_{0}^{0}} Q$, we have $C_{P / Q}\left(v_{4} Q\right)=\left\langle h_{2}(-1)\right\rangle X_{01_{0}^{0}} Q$. We then see from the precise information on centralizers in the appendix that $C_{P}\left(v_{4}\right)=$ $U_{4} \cdot S_{3}$ as required.

Lemma 5.19 If $G=D_{4}$ then $C_{G}\left(v_{i}\right)$ is as given in Table 8 for $i=5$.

Proof Here $u$ is distinguished in $G$ with $C_{P}(u)=C_{Q}(u)$ by [11, Table 8.5a]; so Table 6 shows that $C_{P}\left(v_{5}\right)=U_{2}$ as required. 


\subsection{Completion of proofs}

We can now complete the proof of Theorems 1.4 and 1.5. We have shown that the information in Tables 7 and 8 is correct. Since the entries in the fourth columns of these tables are all different, it is clear that the elements $v_{i}$ represent distinct conjugacy classes in $G\langle\tau\rangle$. What remains is to show that the $v_{i}$ form a complete set of conjugacy class representatives for the outer unipotent classes in $G \tau$, and to verify that the information provided in Tables 9 and 10 is correct. An elementary calculation gives an alternative proof of Theorem 1.1 for these cases. In this subsection we shall continue to assume $G$ is adjoint.

Recall that $\sigma$ is the $q$-field morphism of $G$ satisfying $x_{\beta}(c)^{\sigma}=x_{\beta}\left(c^{q}\right)$ for all $\beta \in \Sigma$ and $c \in K$, and $\gamma$ is either $\sigma$ or $\sigma \tau$. From the expressions for the $v_{i}$ in Tables 7 and 8 we see that $\sigma$ stabilizes each $v_{i}$ and hence the corresponding orbit $O_{i}=v_{i}{ }^{G}$. As $v_{i}=g \tau$ for some $g \in G$, we have $O_{i}=O_{i}{ }^{v_{i}}=O_{i}{ }^{\tau}$, and so $\tau$ also stabilizes $O_{i}$ for each $i$. Therefore $\gamma$ stabilizes each $O_{i}$.

We now apply the usual Lang-Steinberg method to $\Omega=O_{1} \cup \cdots \cup O_{n}$, where we set $n$ to be 17 or 5 according as $G=E_{6}$ or $D_{4}$. For each $i \leq n$, we find that $\gamma$ fixes an element $j_{i}$ in $O_{i}$, so that $j_{i} \in G_{\gamma} \tau$; moreover, $O_{i} \cap G_{\gamma} \tau$ is a union of $G_{\gamma}$-orbits, and the number of orbits and their sizes are determined by the action of $\gamma$ on the component group of $C_{G}\left(j_{i}\right) \cong C_{G}\left(v_{i}\right)$. We see from Tables 7 and 8 that this component group is either $1, Z_{2}, S_{3}$ or $S_{4}$; correspondingly, $O_{i} \cap G_{\gamma} \tau$ is a union of $1,2,3$ or 5 orbits.

The number of $G_{\gamma}$-orbits in $\Omega_{\gamma}$ which this yields is 28 or 7 according as $G=E_{6}$ or $D_{4}$; that is, we have 28 or 7 classes of $p$-elements in $G_{\gamma} \tau$. Therefore Lemma 3.3(i) implies that $\Omega_{\gamma}$ is the complete set of outer unipotent elements in $G_{\gamma} \tau$. And as this holds for all $q$, we argue as in the proof of Lemma 3.3(ii) that this forces $\Omega$ to be the complete set of outer unipotent elements in $G \tau$.

Finally, we must verify that the information in the third column of Tables 9 and 10 is correct. This procedure is just the usual Lang-Steinberg approach. Fix $i \leq n$ and consider $C_{i}=C_{G}\left(j_{i}\right)$. If the unipotent radical of this centralizer is $U_{d}$, then the group of fixed points has order $q^{d}$. Moreover, fixed points of $\gamma$ on $R_{i}=C_{i} / R_{u}\left(C_{i}\right)$ are covered by actual fixed points. We must determine the possible actions of $c \gamma$ on $R_{i}$, where $c \in C_{i} / C_{i}{ }^{\circ}$ and $c \gamma$ is a representative of a conjugacy class in $\left(C_{i} / C_{i}{ }^{\circ}\right) \gamma$.

A glance at Tables 7 and 8 shows that the only ambiguity occurs for $G=E_{6}$ and $i=4,5,7$ or 14 , where $R_{i}=D_{3} .2, A_{1} A_{1}, A_{1} A_{1} .2$ or $T_{1} .2$ respectively. Lemmas 5.8, 5.9 and 5.13 show that in the first, third and fourth of these cases, the extra involution induces the full group of outer automorphisms on the connected component of $R_{i}$. Consequently two isomorphism types of fixed points are as indicated in Table 9 . In the remaining case $R_{i}$ is connected and the issue is whether or not $\gamma$ interchanges the $A_{1}$ factors of $R_{i}$. However, if $\gamma$ interchanged these factors, then it would also interchange the classes of 1dimensional tori in their preimages. But we see from the argument in Lemma 5.8 that these tori are conjugate to the maximal tori of $Y_{2}$ and $Y_{3,5}$, so this is impossible. 


\section{Tables}

This section contains a number of tables illustrating our results. Tables $7-10$, referred to in Theorems 1.4 and 1.5, cover the cases $G=E_{6}$ and $D_{4}$. Tables 7 and 8 give precise information on outer unipotent elements in $G \tau$, while Tables 9 and 10 give corresponding information for the finite groups.

Tables 11-16 cover the cases $G=A_{l}$ for $2 \leq l \leq 7$; as elsewhere in this paper we write $n=l+1$, and take $G$ to be simply connected so that $G=$ $S L_{n}(K)$. In each of these tables, for each unipotent element $v \in G \tau$ the second column gives the decomposition $V \downarrow v$ as in (1), where $V=V_{2 n}(K)$ is the corresponding symplectic module, and the first column gives the Jordan form of $u=v^{2}$ on $V_{n}(K)$. The third column then gives the dimension of $C=C_{G}(v)$, the fourth gives the reductive part $C / R_{u}(C)$ of $C$, and the last gives the values of the $\epsilon$-function of [16] on the sizes of the Jordan blocks (see the preamble to Lemma 4.2). 
Table 7: Unipotent classes in $G \tau=E_{6} \tau$

\begin{tabular}{|c|c|c|c|}
\hline$\left(v_{i}^{2}\right)^{G}$ & $v_{i}$ & $v_{i}^{E_{7}}$ & $C_{G}\left(v_{i}\right)$ \\
\hline 1 & $\begin{array}{l}v_{1}=\tau \\
v_{2}=\tau u_{12321}\end{array}$ & $\begin{array}{l}\left(A_{1}^{3}\right)^{\prime \prime} \\
A_{1}{ }^{4}\end{array}$ & $\begin{array}{l}F_{4} \\
U_{15} C_{3}\end{array}$ \\
\hline$A_{1}$ & $v_{3}=\tau u_{01210} u_{11111}$ & $A_{2} A_{1}{ }^{3}$ & $U_{14} G_{2}$ \\
\hline$A_{1}{ }^{2}$ & $\begin{array}{l}v_{4}=\tau u_{12211} \\
v_{5}=\tau u_{01210} u_{12211} \\
v_{6}=\tau u_{01100} u_{00110} u_{01210} u_{11111} u_{12211}\end{array}$ & $\begin{array}{l}\left(A_{3} A_{1}\right)^{\prime \prime} \\
A_{3} A_{1}{ }^{2} \\
A_{3} A_{1}{ }^{2}\end{array}$ & $\begin{array}{l}U_{15} D_{3} .2 \\
U_{18} A_{1} A_{1} \\
U_{19} A_{1}\end{array}$ \\
\hline$A_{2}$ & $v_{7}=\tau s_{1} s_{6} s_{4} u_{11111}$ & $D_{4} A_{1}$ & $U_{10} A_{1} A_{1} .2$ \\
\hline$A_{1}^{3}$ & $v_{8}=\tau u_{00100} u_{11111} u_{12210}$ & $A_{3} A_{2} A_{1}$ & $U_{15} A_{1}$ \\
\hline$A_{2}^{2}$ & 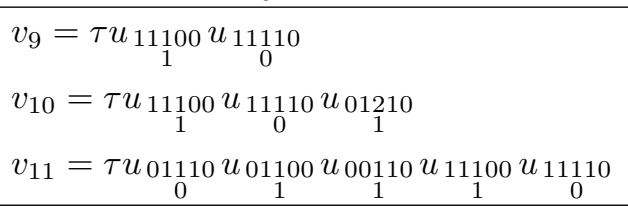 & $\begin{array}{l}\left(A_{5}\right)^{\prime \prime} \\
A_{5} A_{1} \\
D_{6}\left(a_{2}\right)\end{array}$ & $\begin{array}{l}U_{8} G_{2} \\
U_{13} A_{1} \\
U_{11} A_{1} .2\end{array}$ \\
\hline$A_{4}$ & $\begin{array}{l}v_{12}=\tau s_{4} u_{11100} u_{01100} \\
v_{13}=\tau s_{4} u_{11100} u_{01100} u_{01110} \\
0\end{array}$ & $\begin{array}{l}D_{6} \\
D_{6}\end{array}$ & $\begin{array}{l}U_{7} A_{1} .2 \\
U_{8}\end{array}$ \\
\hline$D_{4}\left(a_{1}\right)$ & $v_{14}=\tau s_{1} s_{6} s_{2} u_{00100}^{0} u_{01110}^{010}$ & $D_{5} A_{1}$ & $U_{9} T_{1} .2$ \\
\hline$A_{2}^{2} A_{1}$ & $v_{15}=\tau u_{11100} u_{11110} u_{01110} u_{0}^{00100}$ & $E_{7}\left(a_{5}\right)$ & $U_{12} \cdot S_{4}$ \\
\hline$E_{6}\left(a_{3}\right)$ & $v_{16}=\tau s_{2} s_{3} s_{5} u_{10000} u_{00001} u_{00100}$ & $E_{7}\left(a_{2}\right)$ & $U_{6} .2$ \\
\hline$E_{6}\left(a_{1}\right)$ & $v_{17}=\tau s_{4} u_{10000} u_{00000} u_{01000}$ & $E_{7}$ & $U_{4} .2$ \\
\hline
\end{tabular}

Table 8: Unipotent classes in $G \tau=D_{4} \tau$

\begin{tabular}{|l|l|l|l|}
\hline$\left(v_{i}^{2}\right)^{G}$ & $v_{i}$ & $v_{i}^{F_{4}}$ & $C_{G}\left(v_{i}\right)$ \\
\hline 1 & $v_{1}=\tau$ & $\tilde{A}_{2}$ & $G_{2}$ \\
& $v_{2}=\tau u_{12} 1$ & $\tilde{A}_{2} A_{1}$ & $U_{5} A_{1}$ \\
\hline$A_{1}^{3}$ & $v_{3}=\tau u_{11_{0}^{1}}$ & $C_{3}$ & $U_{3} A_{1}$ \\
& $v_{4}=\tau u_{11_{0}^{1}} u_{01_{0}^{0}}$ & $F_{4}\left(a_{2}\right)$ & $U_{4} \cdot S_{3}$ \\
\hline$D_{4}\left(a_{1}\right)$ & $v_{5}=\tau u_{10_{0}^{0}} u_{01_{0}^{0}}$ & $F_{4}$ & $U_{2}$ \\
\hline
\end{tabular}


Table 9: Classes of 2-elements in $G_{\gamma} \tau, G=E_{6}$

\begin{tabular}{|l|c|l|}
\hline Class rep. in $G \tau$ & no. of $G_{\gamma^{-}}$classes & centralizer orders in $G_{\gamma}$ \\
\hline \hline$v_{1}$ & 1 & $\left|F_{4}(q)\right|$ \\
$v_{2}$ & 1 & $q^{15}\left|C_{3}(q)\right|$ \\
$v_{3}$ & 1 & $q^{14}\left|G_{2}(q)\right|$ \\
$v_{4}$ & 2 & $2 q^{15}\left|A_{3}(q)\right|, 2 q^{15}\left|{ }^{2} A_{3}(q)\right|$ \\
$v_{5}$ & 1 & $q^{18}\left|A_{1}(q)\right|\left|A_{1}(q)\right|$ \\
$v_{6}$ & 1 & $q^{19}\left|A_{1}(q)\right|$ \\
$v_{7}$ & 2 & $2 q^{10}\left|A_{1}(q)\right|\left|A_{1}(q)\right|, 2 q^{10}\left|A_{1}\left(q^{2}\right)\right|$ \\
$v_{8}$ & 1 & $q^{15}\left|A_{1}(q)\right|$ \\
$v_{9}$ & 1 & $q^{8}\left|G_{2}(q)\right|$ \\
$v_{10}$ & 1 & $q^{13}\left|A_{1}(q)\right|$ \\
$v_{11}$ & 2 & $2 q^{11}\left|A_{1}(q)\right|, 2 q^{11}\left|A_{1}(q)\right|$ \\
$v_{12}$ & 2 & $2 q^{7}\left|A_{1}(q)\right|, 2 q^{7}\left|A_{1}(q)\right|$ \\
$v_{13}$ & 1 & $q^{8}$ \\
$v_{14}$ & 2 & $2 q^{9}(q-1), 2 q^{9}(q+1)$ \\
$v_{15}$ & 5 & $24 q^{12}, 8 q^{12}, 4 q^{12}, 4 q^{12}, 3 q^{12}$ \\
$v_{16}$ & 2 & $2 q^{6}, 2 q^{6}$ \\
$v_{17}$ & 2 & $2 q^{4}, 2 q^{4}$ \\
\hline
\end{tabular}

Table 10: Classes of 3-elements in $G_{\gamma} \tau, G=D_{4}$

\begin{tabular}{|l|c|l|}
\hline Class rep. in $G \tau$ & no. of $G_{\gamma}$-classes & centralizer orders in $G_{\gamma}$ \\
\hline \hline$v_{1}$ & 1 & $\left|G_{2}(q)\right|$ \\
$v_{2}$ & 1 & $q^{5}\left|A_{1}(q)\right|$ \\
$v_{3}$ & 1 & $q^{3}\left|A_{1}(q)\right|$ \\
$v_{4}$ & 3 & $6 q^{4}, 3 q^{4}, 2 q^{4}$ \\
$v_{5}$ & 1 & $q^{2}$ \\
\hline
\end{tabular}


Table 11: Unipotent classes in $G \tau=A_{2} \tau$

\begin{tabular}{|l|l|l|l|l|}
\hline$u$ & $v$ & $\operatorname{dim} C$ & $C / R_{u}(C)$ & $\epsilon$-function \\
\hline$J_{1}{ }^{3}$ & $W(2)+V(2)$ & 3 & $S p_{2}$ & $1 \mapsto 1$ \\
$J_{3}$ & $V(6)$ & 1 & 2 & $3 \mapsto 1$ \\
\hline
\end{tabular}

Table 12: Unipotent classes in $G \tau=A_{3} \tau$

\begin{tabular}{|l|l|l|l|l|}
\hline$u$ & $v$ & $\operatorname{dim} C$ & $C / R_{u}(C)$ & $\epsilon$-function \\
\hline$J_{1}{ }^{4}$ & $W(2)^{2}$ & 10 & $S p_{4}$ & $1 \mapsto 0$ \\
& $W(2)+V(2)^{2}$ & 6 & $S p_{2}$ & $1 \mapsto 1$ \\
$J_{2}{ }^{2}$ & $W(4)$ & 4 & $O_{2}$ & $2 \mapsto \omega$ \\
$J_{3}, J_{1}$ & $V(6)+V(2)$ & 2 & 1 & $3 \mapsto 1,1 \mapsto 1$ \\
\hline
\end{tabular}

Table 13: Unipotent classes in $G \tau=A_{4} \tau$

\begin{tabular}{|l|l|l|l|l|}
\hline$u$ & $v$ & $\operatorname{dim} C$ & $C / R_{u}(C)$ & $\epsilon$-function \\
\hline$J_{1}{ }^{5}$ & $W(2)^{2}+V(2)$ & 10 & $S p_{4}$ & $1 \mapsto 1$ \\
$J_{2}{ }^{2}, J_{1}$ & $W(4)+V(2)$ & 6 & $S p_{2}$ & $2 \mapsto \omega, 1 \mapsto 1$ \\
$J_{3}, J_{1}{ }^{2}$ & $V(6)+W(2)$ & 6 & $S p_{2} \times 2$ & $3 \mapsto 1,1 \mapsto 0$ \\
& $V(6)+V(2)^{2}$ & 4 & 1 & $3 \mapsto 1,1 \mapsto 1$ \\
$J_{5}$ & $V(10)$ & 2 & 2 & $5 \mapsto 1$ \\
\hline
\end{tabular}

Table 14: Unipotent classes in $G \tau=A_{5} \tau$

\begin{tabular}{|l|l|l|l|l|}
\hline$u$ & $v$ & $\operatorname{dim} C$ & $C / R_{u}(C)$ & $\epsilon$-function \\
\hline$J_{1}{ }^{6}$ & $W(2)^{3}$ & 21 & $S p_{6}$ & $1 \mapsto 0$ \\
& $W(2)^{2}+V(2)^{2}$ & 15 & $S p_{4}$ & $1 \mapsto 1$ \\
$J_{2}{ }^{2}, J_{1}{ }^{2}$ & $W(4)+W(2)$ & 11 & $S p_{2} \times O_{2}$ & $2 \mapsto \omega, 1 \mapsto 0$ \\
& $W(4)+V(2)^{2}$ & 9 & $S p_{2}$ & $2 \mapsto \omega, 1 \mapsto 1$ \\
$J_{3}, J_{1}{ }^{3}$ & $V(6)+W(2)+V(2)$ & 7 & $S p_{2}$ & $3 \mapsto 1,1 \mapsto 1$ \\
$J_{3}{ }^{2}$ & $W(6)$ & 7 & $S p_{2}$ & $3 \mapsto 0$ \\
& $V(6)^{2}$ & 5 & 2 & $3 \mapsto 1$ \\
$J_{5}, J_{1}$ & $V(10)+V(2)$ & 3 & 2 & $5 \mapsto 1,1 \mapsto 1$ \\
\hline
\end{tabular}


Table 15: Unipotent classes in $G \tau=A_{6} \tau$

\begin{tabular}{|l|l|l|l|l|}
\hline$u$ & $v$ & $\operatorname{dim} C$ & $C / R_{u}(C)$ & $\epsilon$-function \\
\hline$J_{1}{ }^{7}$ & $W(2)^{3}+V(2)$ & 21 & $S p_{6}$ & $1 \mapsto 1$ \\
$J_{2}{ }^{2}, J_{1}{ }^{3}$ & $W(4)+W(2)+V(2)$ & 13 & $S p_{2} \times S p_{2}$ & $2 \mapsto \omega, 1 \mapsto 1$ \\
$J_{3}, J_{1}{ }^{4}$ & $V(6)+W(2)^{2}$ & 15 & $S p_{4} \times 2$ & $3 \mapsto 1,1 \mapsto 0$ \\
& $V(6)+W(2)+V(2)^{2}$ & 11 & $S p_{2}$ & $3 \mapsto 1,1 \mapsto 1$ \\
$J_{3}, J_{2}{ }^{2}$ & $V(6)+W(4)$ & 9 & $S p_{2} \times 2$ & $3 \mapsto 1,2 \mapsto \omega$ \\
$J_{3}{ }^{2}, J_{1}$ & $W(6)+V(2)$ & 9 & $S p_{2}$ & $3 \mapsto 0,1 \mapsto 1$ \\
& $V(6)^{2}+V(2)$ & 7 & 1 & $3 \mapsto 1,1 \mapsto 1$ \\
$J_{5}, J_{1}{ }^{2}$ & $V(10)+W(2)$ & 7 & $S p_{2} \times 2$ & $5 \mapsto 1,1 \mapsto 0$ \\
& $V(10)+V(2)^{2}$ & 5 & 2 & $5 \mapsto 1,1 \mapsto 1$ \\
$J_{7}$ & $V(14)$ & 3 & 2 & $7 \mapsto 1$ \\
\hline
\end{tabular}

Table 16: Unipotent classes in $G \tau=A_{7} \tau$

\begin{tabular}{|l|l|l|l|l|}
\hline$u$ & $v$ & $\operatorname{dim} C$ & $C / R_{u}(C)$ & $\epsilon$-function \\
\hline$J_{1}{ }^{8}$ & $W(2)^{4}$ & 36 & $S p_{8}$ & $1 \mapsto 0$ \\
& $W(2)^{3}+V(2)^{2}$ & 28 & $S p_{6}$ & $1 \mapsto 1$ \\
$J_{2}{ }^{2}, J_{1}{ }^{4}$ & $W(4)+W(2)^{2}$ & 22 & $S p_{2} \times O_{2}$ & $2 \mapsto \omega, 1 \mapsto 0$ \\
& $W(4)+W(2)+V(2)^{2}$ & 18 & $S p_{2} \times S p_{2}$ & $2 \mapsto \omega, 1 \mapsto 1$ \\
$J_{2}{ }^{4}$ & $W(4)^{2}$ & 16 & $O_{4}$ & $2 \mapsto \omega$ \\
$J_{3}, J_{1}{ }^{5}$ & $V(6)+W(2)^{2}+V(2)$ & 16 & $S p_{4}$ & $3 \mapsto 1,1 \mapsto 1$ \\
$J_{3}, J_{2}{ }^{2}, J_{1}$ & $V(6)+W(4)+V(2)$ & 12 & $S p_{2}$ & $3 \mapsto 1,2 \mapsto \omega, 1 \mapsto 1$ \\
$J_{3}{ }^{2}, J_{1}{ }^{2}$ & $W(6)+W(2)$ & 14 & $S p_{2} \times S p_{2}$ & $3 \mapsto 0,1 \mapsto 0$ \\
& $W(6)+V(2)^{2}$ & 12 & $S p_{2}$ & $3 \mapsto 0,1 \mapsto 1$ \\
& $V(6)^{2}+W(2)$ & 12 & $S p_{2} \times 2$ & $3 \mapsto 1,1 \mapsto 0$ \\
$J_{4}{ }^{2}$ & $V(6)^{2}+V(2)^{2}$ & 10 & 1 & $3 \mapsto 1,1 \mapsto 1$ \\
$J_{5}, J_{1}{ }^{2}$ & $W(8)$ & 8 & $O_{2}$ & $4 \mapsto \omega$ \\
$J_{5}, J_{3}$ & $V(10)+W(2)+V(2)$ & 8 & $S p_{2} \times 2$ & $5 \mapsto 1,1 \mapsto 1$ \\
$J_{7}, J_{1}$ & $V(10)+V(6)$ & 6 & 2 & $5 \mapsto 1,3 \mapsto 1$ \\
\hline
\end{tabular}




\section{Appendix: Explicit $U$-centralizers}

We have seen that the determination of the centralizers $C_{G}\left(v_{i}\right)$ in subsection 5.3 frequently uses knowledge of the subgroups $C_{Q}\left(v_{i}\right)$ and $C_{U}\left(v_{i}\right)$. The structure of these groups is given in Tables 5 and 6 , but in one or two places more detailed information is required. We conclude by providing explicit expressions for the groups $C_{U}\left(v_{i}\right)$; Tables 5 and 6 summarize the results presented here.

We begin with a brief comment on the structure constants in $G$. Since all roots in the root system $\Sigma$ are long, the only non-trivial Chevalley commutator relations are of the form $\left[x_{\alpha}\left(t_{1}\right), x_{\beta}\left(t_{2}\right)\right]=x_{\alpha+\beta}\left(N_{\alpha, \beta} t_{1} t_{2}\right)$, in which the structure constant $N_{\alpha, \beta}$ is \pm 1 . If $G=E_{6}$ there is no ambiguity, since we are working in characteristic 2; however if $G=D_{4}$ we must specify the choices made. We have taken $N_{\alpha, \beta}=1$ for the following ordered pairs of positive roots $(\alpha, \beta)$ :

$$
\begin{aligned}
& \left(10_{0}^{0}, 01{ }_{0}^{0}\right),\left(00 \frac{1}{0}, 010\right),\left(00{ }_{1}^{0}, 010\right),\left(11_{0}^{0}, 001\right),\left(01{ }_{0}^{1}, 00 \quad{ }_{1}^{0}\right),\left(01{ }_{1}^{0}, 10_{0}^{0}\right) \text {, } \\
& \left(01{ }_{0}^{1}, 10_{0}^{0}\right),\left(01{ }_{1}^{0}, 00 \begin{array}{l}
1 \\
0
\end{array}\right),\left(11_{0}^{0}, 00{ }_{1}^{0}\right),\left(10_{0}^{0}, 01 \frac{1}{1}\right),\left(00{ }_{0}^{1}, 11{ }_{1}^{0}\right),\left(00{ }_{1}^{0}, 11{ }_{0}^{1}\right) \text {, } \\
& \left(01{ }_{1}^{1}, 11_{0}^{0}\right),\left(11_{1}^{0}, 01{ }_{0}^{1}\right),\left(11{ }_{0}^{1}, 0101\right),\left(01{ }_{0}^{0}, 11{ }_{1}^{1}\right) \text {. }
\end{aligned}
$$

The structure constants were calculated using [3, Proposition 4.2.2]; as can be seen, for all $\alpha, \beta \in \Sigma^{+}$we have $N_{\alpha^{\tau}, \beta^{\tau}}=N_{\alpha, \beta}$. Since $x_{\alpha}(t)^{\tau}=x_{\alpha^{\tau}}(t)$ for $\alpha \in \Pi$, by taking commutators we see that the same is true for all $\alpha \in \Sigma^{+}$.

We now describe how we use a computer to obtain the groups $C_{U}\left(v_{i}\right)$. We begin with the element $v_{i}$ and write it as $\tau s x$, where $x \in U$ and $s \in N_{G}\left(T_{G}\right)$ (so that $s$ corresponds to an element of the Weyl group); usually $s=1$, but in some instances in $G=E_{6}$ it is a product of reflections in mutually orthogonal simple roots. We also take a 'generic' element $g=\prod_{\beta \in \Xi} x_{\beta}\left(\kappa_{\beta}\right)$ of $U$, where $\Xi=\Sigma^{+} \cap\left(\Sigma^{+}\right)^{s}$, and the various $\kappa_{\beta}$ are regarded as indeterminates; we order the roots in $\Xi$ so that the roots outside $Q$ precede those inside $Q$.

We form the commutator $\left[g, v_{i}\right]=g^{-1} \cdot x^{-1} \cdot g^{\tau s} \cdot x$, which we treat as a sequence of root elements corresponding to positive roots. This sequence is then passed through a simplifying program which reduces it to a canonical form; in this form the roots are taken in a fixed order compatible with height. If this canonical form is not the identity, we choose a root for which the coefficient is non-zero, and seek to make it zero by writing one of the $\kappa_{\beta}$ in the expression for $g$ in terms of the remaining indeterminates. This gives a modified sequence for the commutator, which we pass through the simplifying program again, and the resulting canonical form will have fewer non-zero coefficients. We continue in this way until the canonical form has been reduced to the identity; at this point, the expression for $g$ gives the form of an arbitrary element of $C_{U}\left(v_{i}\right)$.

The expressions obtained are given in Tables 17 and 18. Our notation in these tables is as follows. We write $c_{j}$ and $t_{j}$ for arbitrary elements of $K$, with the exception that in two instances in Table 17 a relation of the form $t_{j}{ }^{2}=t_{k}{ }^{2}+t_{k}$ holds. If it appears, $\zeta$ stands for an element of a finite field $\mathbb{F}$ (usually $\mathbb{F}=\mathbb{F}_{p}$, but in one instance in $E_{6}$ we have $\mathbb{F}=\mathbb{F}_{4}$ ). If all $c_{j}$ are set to be 0 , the resulting expression gives a typical element of $C_{Q}\left(v_{i}\right)$. 
Table 17: Explicit $U$-centralizers for $G=E_{6}$

\begin{tabular}{|c|c|}
\hline$i$ & $C_{U}\left(v_{i}\right)$ \\
\hline 1,2 & 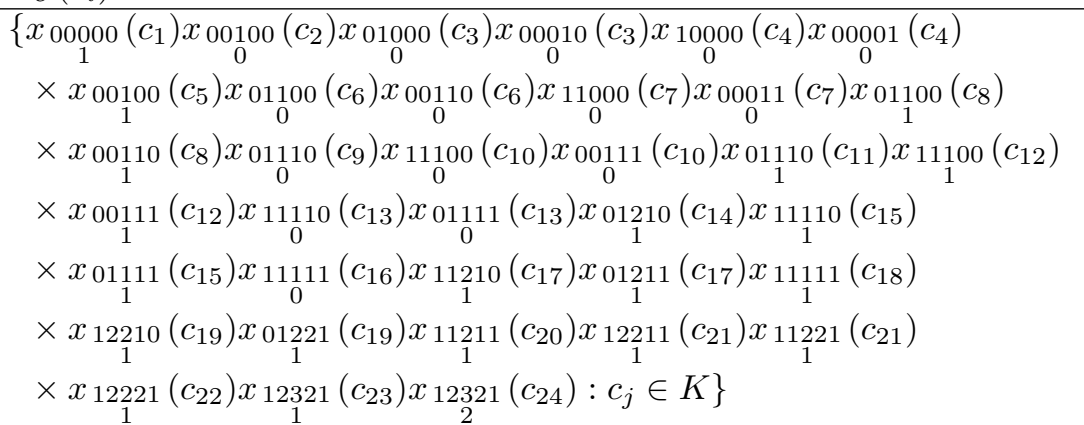 \\
\hline 3 & 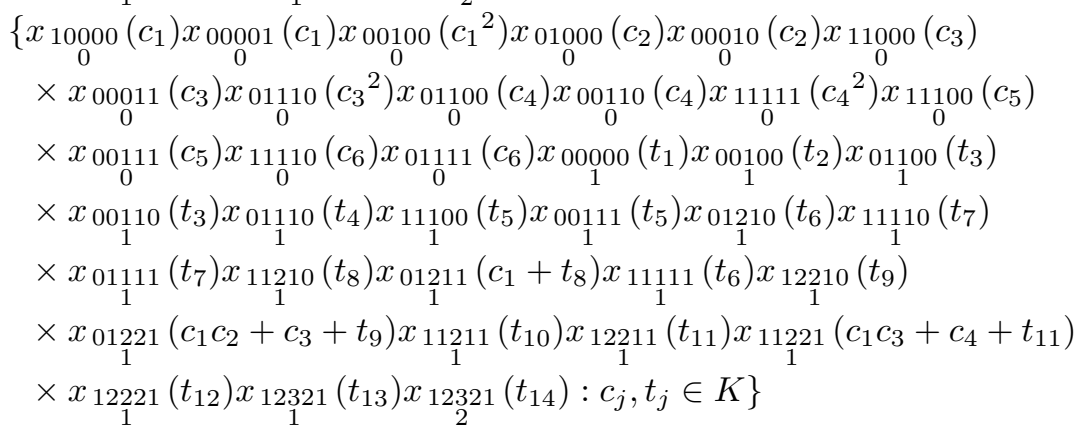 \\
\hline 4 & 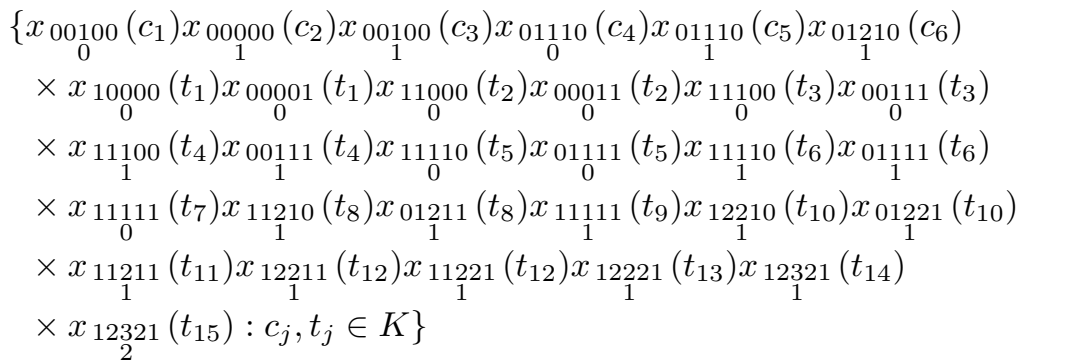 \\
\hline 5 & 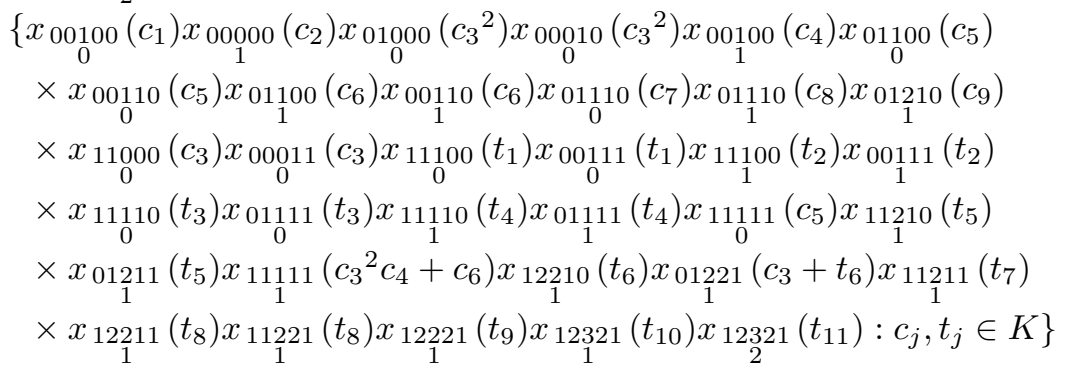 \\
\hline
\end{tabular}


Table 17: Explicit $U$-centralizers for $G=E_{6}$ (continued)

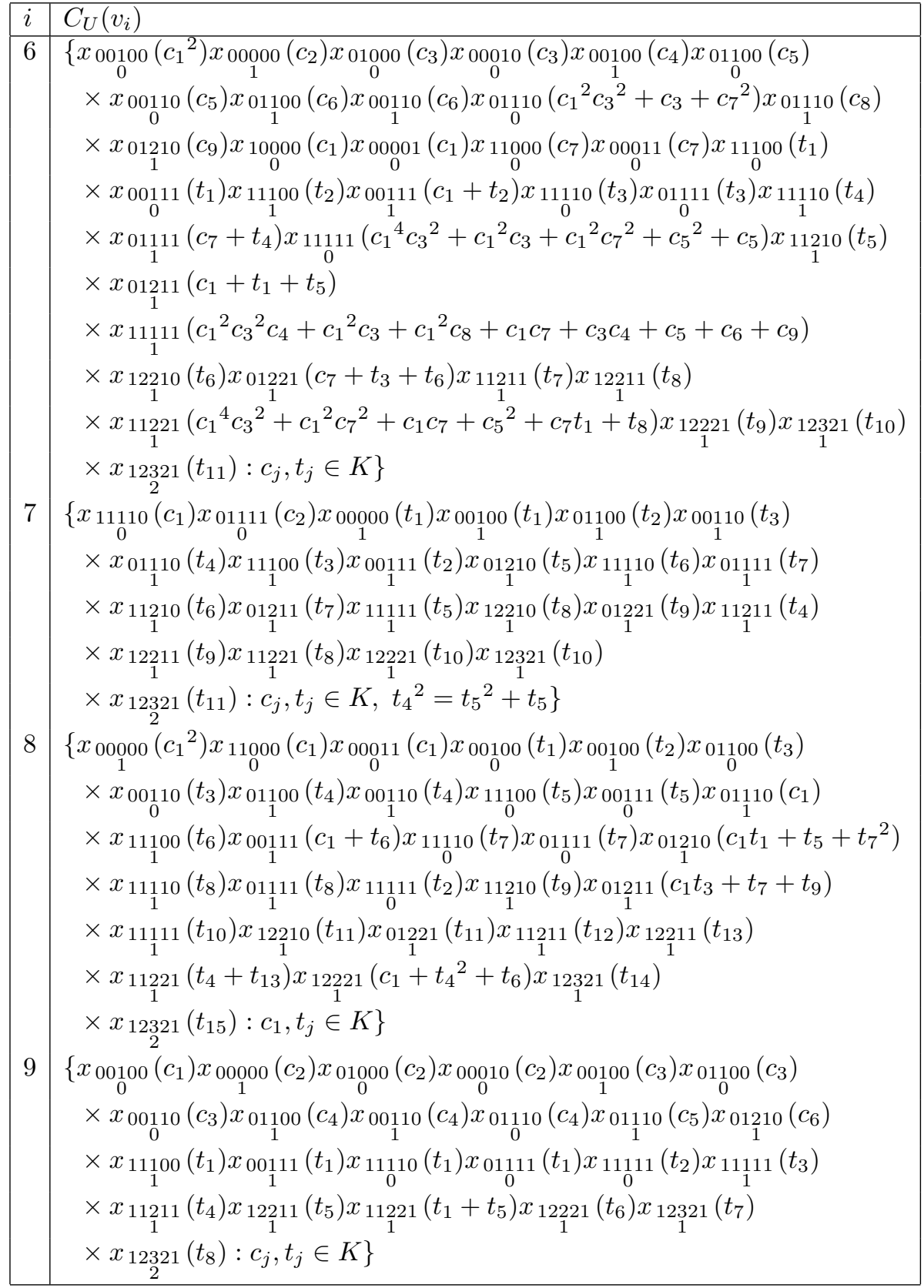


Table 17: Explicit $U$-centralizers for $G=E_{6}$ (continued)

\begin{tabular}{|c|c|}
\hline$i$ & $C_{U}\left(v_{i}\right)$ \\
\hline 10 & $\begin{array}{l}\left\{x_{00100}\left(c_{1}\right) x_{00000}\left(c_{2}\right) x_{01000}\left(c_{2}\right) x_{00010}\left(c_{2}\right) x_{00100}\left(c_{3}\right) x_{01100}\left(c_{3}\right)\right. \\
\quad \times 0 \\
\quad \times x_{00110}\left(c_{3}\right) x_{01100}\left(c_{4}\right) x_{00110}\left(c_{4}\right) x_{01110}\left(c_{4}\right) x_{01110}\left(c_{5}\right) x_{01210}\left(c_{6}\right) \\
\quad \times x_{11100}\left(t_{1}\right) x_{00111}\left(t_{1}\right) x_{11110}\left(t_{1}\right) x_{01111}\left(t_{1}\right) x_{11111}\left(t_{2}\right) x_{11210}\left(t_{2}\right) \\
\quad \times 1 \\
\quad \times x_{01211}\left(t_{2}\right) x_{11111}\left(t_{3}\right) x_{12210}\left(t_{3}\right) x_{01221}\left(t_{3}\right) x_{11211}\left(t_{4}\right) x_{12211}\left(t_{5}\right) \\
\left.\quad \times x_{11221}\left(t_{1}+t_{5}\right) x_{12221}\left(t_{6}\right) x_{12321}\left(t_{7}\right) x_{12321}\left(t_{8}\right): c_{j}, t_{j} \in K\right\}\end{array}$ \\
\hline 11 & $\begin{array}{l}\left\{x_{00100}\left(c_{1}\right) x_{01100}\left(c_{2}\right) x_{00110}\left(c_{2}\right) x_{01110}\left(c_{2}\right) x_{01110}\left(c_{3}\right) x_{01210}\left(c_{4}\right)\right. \\
\quad \times x_{10000}(\zeta) x_{00001}(\zeta) x_{11100}\left(t_{1}\right) x_{00111}\left(\zeta+t_{1}\right) x_{11110}\left(\zeta+t_{1}\right) x_{01111}\left(t_{1}\right) \\
0 \\
0 \\
\quad \times x_{11110}\left(t_{2}\right) x_{01111}\left(t_{2}\right) x_{11111}\left(t_{3}\right) x_{11210}\left(t_{4}\right) x_{01211}\left(t_{4}\right) x_{11111}\left(t_{2}\right) \\
\quad \times x_{12210}\left(t_{1}{ }^{2}+t_{1}\right) x_{01221}\left(t_{1}{ }^{2}+t_{1}\right) x_{11211}\left(t_{4}\right) x_{12211}\left(t_{5}\right) \\
\quad \times 1 \\
\quad \times x_{11221}\left(\zeta t_{1}+t_{1}+t_{3}+t_{5}\right) x_{12221}\left(t_{6}\right) x_{12321}\left(t_{7}\right) \\
\left.\quad \times x_{12321}\left(t_{8}\right): \zeta \in \mathbb{F}_{2}, c_{j}, t_{j} \in K\right\}\end{array}$ \\
\hline 12 & 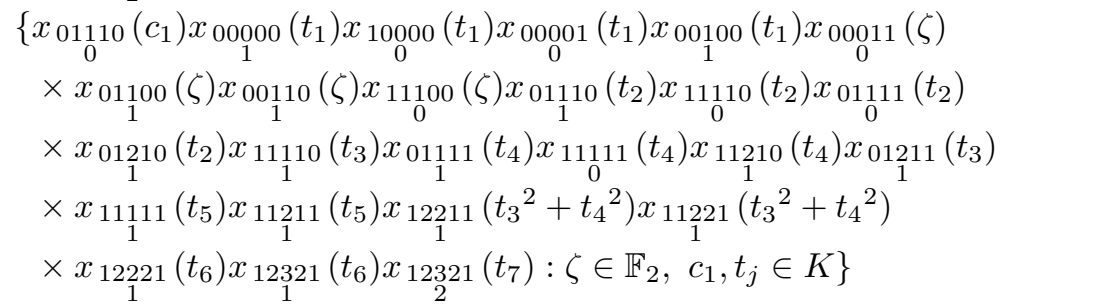 \\
\hline 13 & 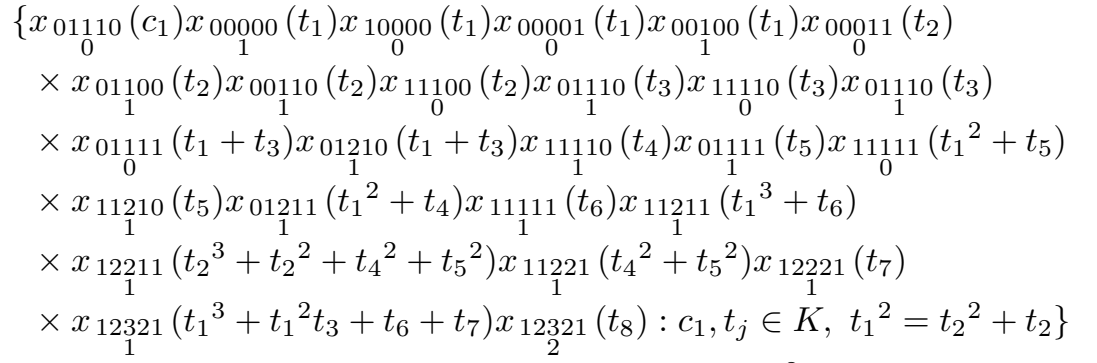 \\
\hline 14 & 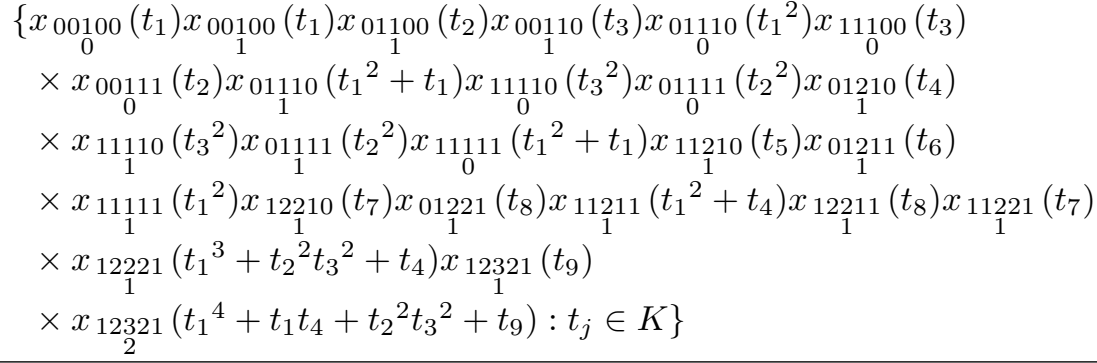 \\
\hline
\end{tabular}


Table 17: Explicit $U$-centralizers for $G=E_{6}$ (continued)

\begin{tabular}{|c|c|}
\hline$i$ & $C_{U}\left(v_{i}\right)$ \\
\hline 15 & 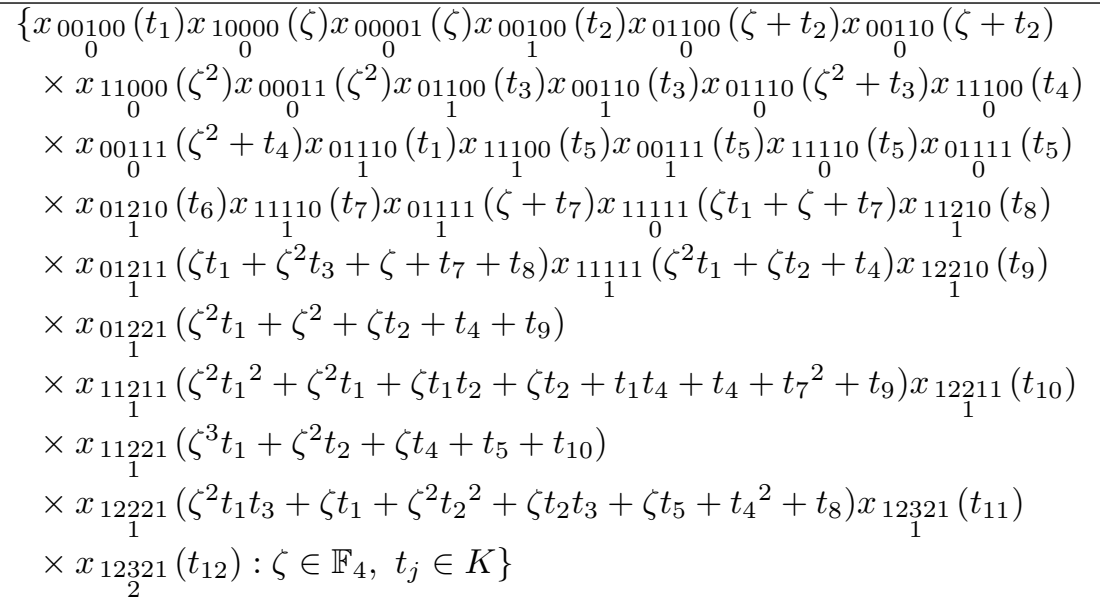 \\
\hline 16 & 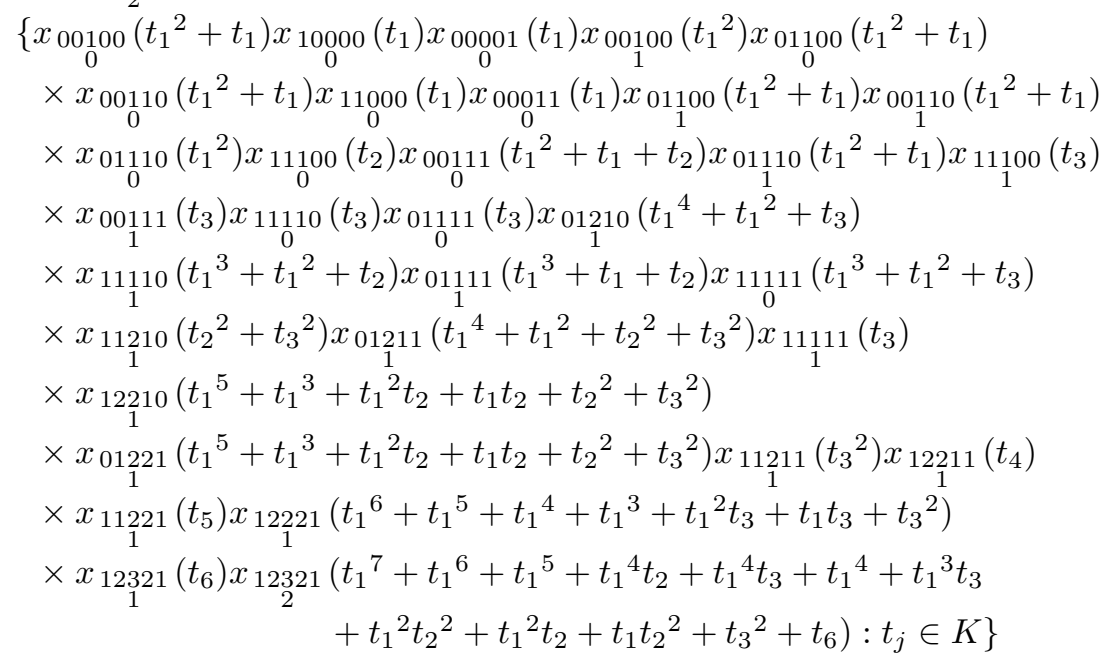 \\
\hline 17 & 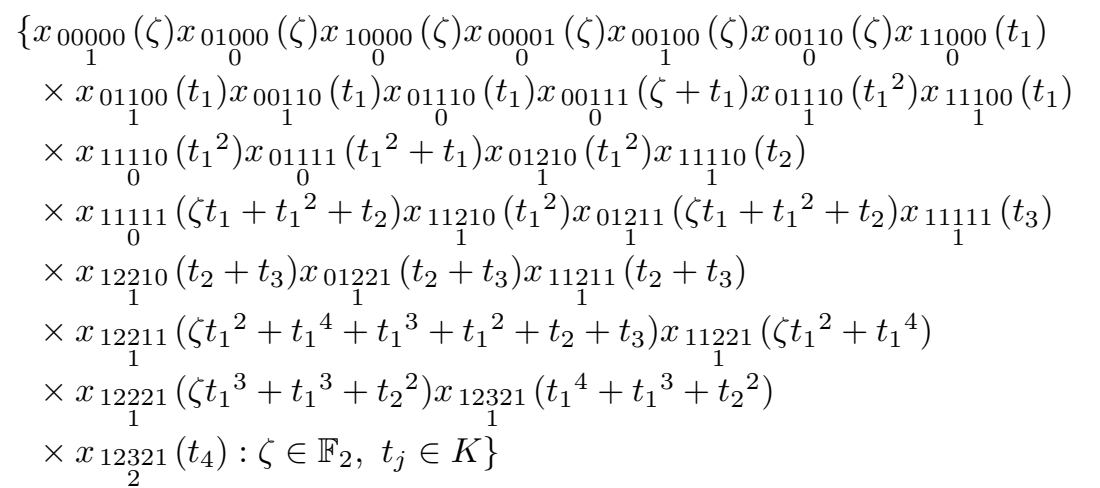 \\
\hline
\end{tabular}


Table 18: Explicit $U$-centralizers for $G=D_{4}$

\begin{tabular}{|c|c|}
\hline$i$ & $C_{U}\left(v_{i}\right)$ \\
\hline 1,2 & $\left\{x_{10_{0}^{0}}\left(c_{1}\right) x_{00}{ }_{0}^{1}\left(c_{1}\right) x_{00_{1}^{0}}\left(c_{1}\right) x_{01_{0}^{0}}\left(c_{2}\right) x_{11_{0}^{0}}\left(c_{3}\right) x_{01_{0}^{1}}\left(c_{3}\right) x_{01_{1}^{0}}\left(c_{3}\right)\right.$ \\
\hline & $\left.\times x_{11_{0}^{1}}\left(c_{4}\right) x_{01_{1}^{1}}\left(c_{4}\right) x_{11_{1}^{0}}\left(c_{4}\right) x_{11_{1}^{1}}\left(c_{5}\right) x_{12 \frac{1}{1}}\left(c_{6}\right): c_{j} \in K\right\}$ \\
\hline 3 & $\left\{x_{01_{0}^{0}}\left(c_{1}\right) x_{11_{0}^{1}}\left(t_{1}\right) x_{01_{1}^{1}}\left(t_{1}\right) x_{11_{1}^{0}}\left(t_{1}\right) x_{11_{1}^{1}}\left(t_{2}\right) x_{121_{1}^{1}}\left(t_{3}\right): c_{1}, t_{j} \in K\right\}$ \\
\hline 4 & $\left\{x_{01_{0}^{0}}\left(c_{1}\right) x_{10_{0}^{0}}(\zeta) x_{00_{0}^{1}}(\zeta) x_{00_{1}^{0}}(\zeta) x_{11_{0}^{0}}\left(t_{1}\right) x_{01_{0}^{1}}\left(\zeta+t_{1}\right)\right.$ \\
\hline & $\times x_{011_{1}^{0}}\left(-\zeta+t_{1}\right) x_{11_{0}^{1}}\left(t_{2}\right) x_{01 \frac{1}{1}}\left(\zeta^{2}+t_{2}\right) x_{11_{1}^{0}}\left(-\zeta^{2}+t_{2}\right) x_{11_{1}^{1}}\left(-t_{1}\right)$ \\
\hline & $\left.\times x_{121}^{1}\left(t_{3}\right): \zeta \in \mathbb{F}_{3}, c_{1}, t_{j} \in K\right\}$ \\
\hline 5 & $\left\{x_{10_{0}^{0}}\left(t_{1}\right) x_{00_{0}^{1}}\left(t_{1}\right) x_{00_{1}^{0}}\left(t_{1}\right) x_{11_{0}^{0}}\left(t_{1}\right) x_{01_{0}^{1}}\left(-t_{1}\right) x_{11_{0}^{1}}\left(-t_{1}^{3}\right)\right.$ \\
\hline & $\times x_{011_{1}^{1}}\left(-t_{1}^{3}+t_{1}^{2}\right) x_{11_{1}^{0}}\left(-t_{1}^{3}-t_{1}^{2}-t_{1}\right) x_{11_{1}^{1}}\left(t_{1}^{3}-t_{1}^{2}\right)$ \\
\hline & $\left.\times x_{12}{ }_{1}^{1}\left(t_{2}\right): t_{j} \in K\right\}$ \\
\hline
\end{tabular}




\section{References}

[1] M. Aschbacher and G.M. Seitz, Involutions in Chevalley groups over fields of even order, Nagoya Math. J. 63 (1976), 1-91.

[2] H. Azad, M. Barry and G.M. Seitz, On the structure of parabolic subgroups, Comm. in Alg. 18 (1990), 551-562.

[3] R.W. Carter, Simple Groups of Lie Type, Wiley-Interscience, London (1972).

[4] R.W. Carter, Finite Groups of Lie Type: Conjugacy Classes and Complex Characters, Wiley-Interscience, London (1985).

[5] F. Digne and J. Michel, Groupes reductifs non connexes, Annals Sci. de l'E.N.S. 27 (1994), 345-406.

[6] J. Fulman and R. Guralnick, Bounds on the number and sizes of conjugacy classes in finite groups of Lie type with applications to derangements, Trans. Amer. Math. Soc. 364 (2012), 3023-3070.

[7] D. Gorenstein, R. Lyons and R. Solomon, The Classification of the Finite Simple Groups, Number 3, Amer. Math. Society Surveys and Monographs 40, Number 3 (1998).

[8] R. M. Guralnick, M. W. Liebeck, H.D. Macpherson and G. M. Seitz, Modules for algebraic groups with finitely many orbits on subspaces, J. Algebra 196 (1997), 211-250.

[9] R. Lawther, Jordan block sizes of unipotent elements in exceptional algebraic groups, Comm. Algebra 23 (1995), 4125-4156.

[10] M.W. Liebeck, Subgroups of simple algebraic groups and of related finite and locally finite groups of Lie type, in Finite and locally finite groups (Istanbul, 1994), NATO Adv. Sci. Inst. Ser. C Math. Phys. Sci. 471, Kluwer Acad. Publ., Dordrecht (1995), pp.71-96.

[11] M.W. Liebeck and G.M. Seitz, Unipotent and nilpotent classes in simple algebraic groups and Lie algebras, Amer. Math. Society Surveys and Monographs $\mathbf{1 8 0}$ (2012).

[12] G. Malle, Green functions for groups of type $E_{6}$ and $F_{4}$ in characteristic 2, Comm. in Alg. 21 (1993), 747-798.

[13] G. Malle, Generalized Deligne-Lusztig characters, J. Algebra 159 (1993), 64-97.

[14] G.M. Seitz, The root subgroups for maximal tori in finite groups of Lie type, Pac. J. Math. 106 (1983), 153-244. 
[15] G.M. Seitz, Generation of finite groups of Lie type, Trans. Amer. Math. Soc. 271 (1982), 351-407.

[16] N. Spaltenstein, Classes unipotents et sous-groupes de Borel, Lecture Notes in Math. 946, Springer, Berlin (1982). 